Une nécropole du second âge du fer à Ambrussum, Hérault

\title{
Chapitre 2. Tombes, structures annexes et sol de la nécropole (fouilles 1999 - 2003)
}

DOI : $10.4000 /$ books.pccj. 1273

Éditeur : Publications du Centre Camille Jullian, Éditions Errance

Lieu d'édition : Aix-en-Provence

Année d'édition : 2012

Date de mise en ligne : 13 février 2020

Collection : Bibliothèque d'archéologie méditerranéenne et africaine

EAN électronique : 9782491788001

\section{QbOOOKS}

http://books.openedition.org

Édition imprimée

Date de publication : 15 mars 2012

\section{Référence électronique}

Chapitre 2. Tombes, structures annexes et sol de la nécropole (fouilles 1999 - 2003) In : Une nécropole du second âge du fer à Ambrussum, Hérault [en ligne]. Aix-en-Provence : Publications du Centre Camille Jullian, 2012 (généré le 21 septembre 2021). Disponible sur Internet : <http://books.openedition.org/ pccj/1273>. ISBN : 9782491788001. DOI : https://doi.org/10.4000/books.pccj.1273. 


\section{Chapitre 2}

\section{Tombes, structures annexes et sol de la nécropole (fouilles 1999 - 2003)}

$\mathrm{C}$ e chapitre rassemble, de manière analytique, les données récoltées dans les seize tombes découvertes lors des recherches conduites entre 1999 et 2003, l'examen des deux structures annexes, ainsi que celui des vestiges livrés par le sol de la nécropole.

Toutes les tombes appartiennent à la catégorie des «dépôts secondaires de crémation », selon la terminologie préconisée dans Blaizot dir. 2009, p. 175. Pour chacune, seront successivement examinés l'état des structures, les restes osseux du défunt, la morphologie et le remplissage du loculus, le dispositif de recouvrement, ainsi que le mobilier et les restes osseux animaux que ces structures ont livrés.

Également fouillé dans ce secteur de la nécropole durant ces campagne de recherches, le bûcher funéraire B1, qui nous renseigne sur les modalités de l'étape de la crémation, peut-être en rapport direct avec ces sépultures elles-mêmes, fera l'objet du chapitre suivant.

\section{La tombe T10}

\section{1. État et composantes}

La tombe T10 accueille les restes d'un adulte de sexe non déterminé. Le mobilier associé ne permet pas de préciser ce dernier. Entièrement fouillée, cette sépulture comprend un loculus et les traces d'une structure de recouvrement (fig. 13).

\subsection{Le défunt}

Le défunt est représenté par 190,3 g d'os incinérés. La morphologie des os, en particulier l'épaisseur de la voûte crânienne, ainsi que la fermeture des apex des dents montrent qu'il s'agit d'un adulte. La quasi-totalité des restes provient du loculus (US 17112-17169). Ceux-ci y sont dispersés, hors de tout contenant.

La couleur blanc crayeux révèle une température de crémation supérieure à $650^{\circ}$ selon le barème de E. Bonnucci et G. Grazziani (1975), et les morceaux de diaphyses sont très souvent fissurés en ondes concentriques, indice d'une incinération d'os frais. Le poids d'os déposés ne représente que $8,6 \%$ du poids moyen d'un squelette d'adulte brûlé, 1627,1 g selon McKinley (1993). Les taux d'identification sont de $83 \%$ du poids total des fragments. L'essentiel est constitué par les membres, $88,7 \%$ du poids identifié (poids théorique $62,6 \%$ ). Crâne et tronc sont, par contre, extrêmement sous-représentés, respectivement $6,9 \%$ et $4,4 \%$, au lieu des 20,4 et $17 \%$ attendus (fig. 13, A et B).

Le poids moyen des fragments déterminés, toutes régions anatomiques confondues, est de $0,15 \mathrm{~g}(0,13 \mathrm{~g}$ pour la tête, $0,10 \mathrm{~g}$ pour le tronc et $0,16 \mathrm{~g}$ pour les membres).

\subsection{Le loculus}

Les limites du loculus (US 17112-17169) se discernent mal de l'encaissant. Le plan est grossièrement ovalaire, d'environ $50 \mathrm{~cm}$ sur l'axe NE-SO, pour environ $30 \mathrm{~cm}$ sur l'axe NO-SE. La profondeur n'excède pas $7 \mathrm{~cm}$. Le comblement est formé d'une terre alluviale semblable à celle de la terrasse environnante. Il s'en distingue cependant par des passées cendreuses, des mouchetures de petits morceaux de charbons de bois, de nombreux fragments d'os humains incinérés et 16 petits tessons de vases appartenant probablement à 15 récipients (fig. 13, C).

\section{Mobilier du loculus}

Ce matériel consiste en tessons isolés d'environ 15 récipients céramiques :

-1 tesson de vase à vernis noir de l'atelier de Rosas, de forme non déterminable ; 


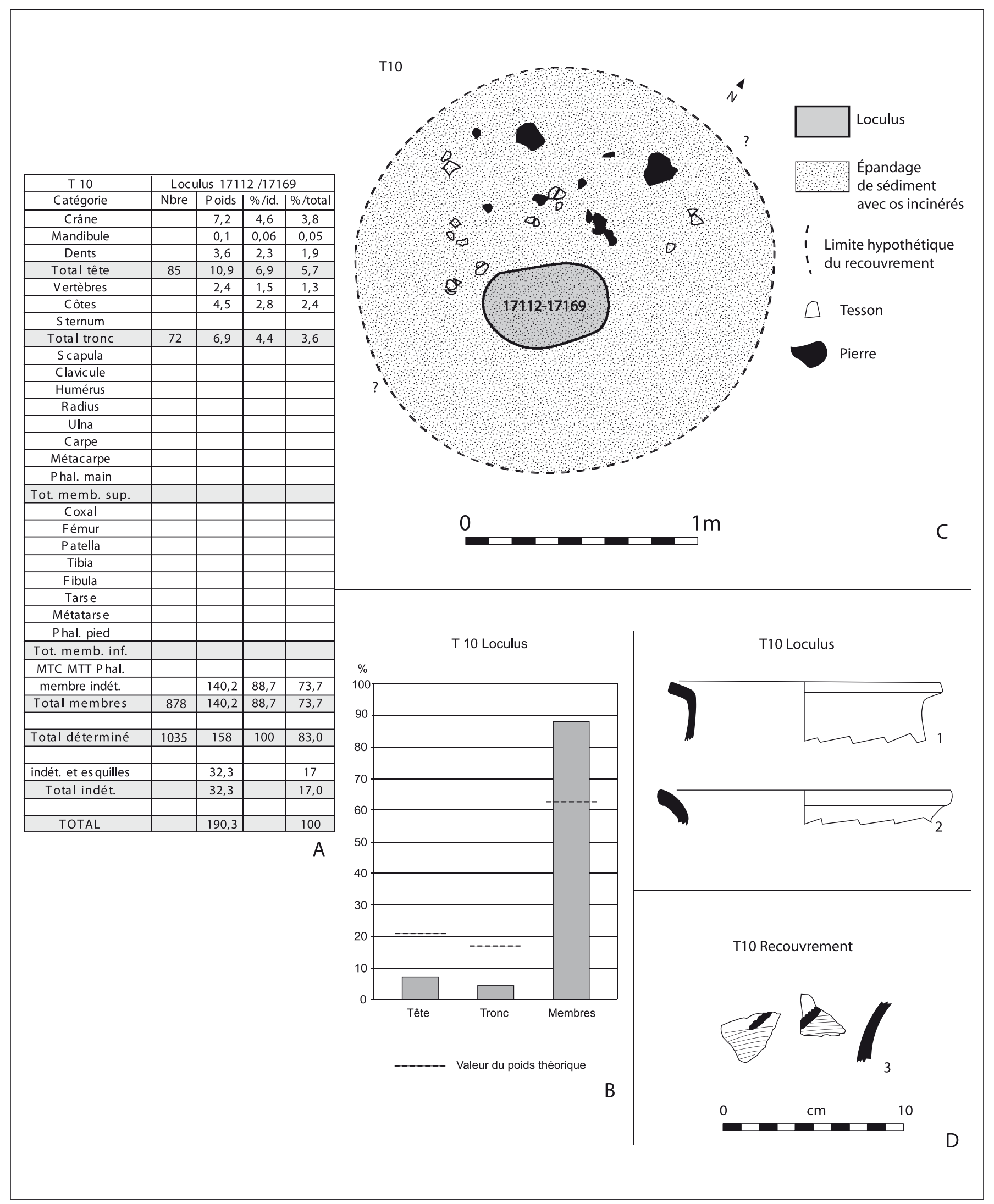

Fig. 13. Tombe T10. A : tableau de détermination des os humains (poids en grammes; calcul des $\%$ sur la masse). $B$ : répartition du poids des os par grandes régions anatomiques (\% par rapport au total déterminé). C : plan de la tombe. $\mathrm{D}:$ mobilier (1-3 : céramique non tournée). 
- 1 tesson d'urne non tournée de forme probable CNT-LOR U5d : col tronconique rentrant, bord déversé formant méplat vers l'intérieur, lèvre aplatie (fig. 13, $\left.\mathrm{n}^{\circ} 1\right)$;

- 2 tessons de bord d'une autre urne non tournée de forme probable CNT-LOR U5d : bord déversé à lèvre aplatie (fig. 13, $\mathrm{n}^{\circ} 2$ ) ;

- 12 tessons ou esquilles de panses de plusieurs vases non tournés (12 exemplaires ?), urnes et coupes (non dessinés).

Os d'animaux (détermination A. Gardeisen) :

- 5 fragments d'extrémité distale de membre antérieur d'un capriné jeune, non brûlée, pouvant provenir d'une portion d'animal incomplètement prélevée (extrémité distale des phalanges I et II, juvénile ; extrémité proximale des phalanges II (non soudée) et III (axiale et abaxiale) ; corps de scaphoïde, juvénile) ;

-1 fragment d'apophyse articulaire de vertèbre cervicale de capriné ;

-1 fragment de côte (cf. bœuf) ;

- Indéterminés : 17 esquilles brûlées et 11 esquilles non brûlées ;

- Malacofaune : gastéropodes blancs de petite taille.

\subsection{La structure de recouvrement}

Les vestiges de la superstructure qui recouvrait ce loculus sont marqués par plusieurs indices. Une portion d'urne non tournée cassée sur place et quelques petites pierres calcaires reposent à plat sur le sol de la nécropole entourant le loculus à l'ouest et au nord. Quelques très rares fragments d'os humains incinérés et quelques charbons de bois sont dispersés dans les deux ou trois centimètres de terre alluviale surmontant le comblement du loculus et le sol aux environs immédiats, et recouvrant les pierres et les tessons. La limite de cette structure est imprécise, mais les vestiges qui attestent ce recouvrement sont présents jusqu'à un mètre de distance du loculus, ce qui lui confère un diamètre de l'ordre de $2 \mathrm{~m}$ (fig. 13, C).

\section{Mobilier de la structure de recouvrement}

- portion de la panse d'une urne non tournée du type ovoïde (23 tessons non jointifs, représentant environ $10 \%$ du récipient); peignage horizontal ; sur l'épaule, décor de grandes impressions au peigne, obliques (fig. 13, $\mathrm{n}^{\circ} 3$ ) ;

-1 tesson de col d'urne non tournée, qui pourrait appartenir à l'exemplaire précédent.

\section{La tombe T11}

\section{1. État et composantes de la tombe}

La tombe 11 abrite un seul défunt incinéré, un adulte de sexe non déterminable, mais la composition du mobilier (voir infra) pourrait cependant signaler une femme.

Cette sépulture a pu être intégralement fouillée; elle comprend un loculus et une structure de recouvrement s'étendant largement tout autour (fig. 14 à 17).

\subsection{Le défunt}

Le défunt est représenté par 284,2 g d'os brûlés. D'après la morphologie des os et la fermeture de l'apex des deux racines de dents conservées, il s'agit d'un adulte. Un fragment crânien dont la suture n'est pas synostosée, sur aucune des deux faces, semble indiquer un adulte jeune. Le sexe anthropologique ne peut pas être précisé.

Les pièces osseuses sont réparties dans le loculus $(183,7 \mathrm{~g})$ et dans les vestiges du dispositif de recouvrement $(100,5 \mathrm{~g})$, et les deux lots ne montrent ni doublets et ni pièces morphologiquement incompatibles.

Dans les deux cas, les restes osseux présentent le même aspect : ils sont de taille très réduite ; les morceaux de diaphyses sont très souvent fissurés en ondes concentriques, indice d'une incinération d'os frais ; tous ont une couleur blanc crayeux qui révèle une température de crémation supérieure à $650^{\circ}$ selon le barème de E. Bonnucci et G. Grazziani (1975).

Dans le loculus, les os sont dispersés, hors de tout contenant, et si aucune disposition particulière des fragments n'a été perçue, ils sont cependant plus abondants dans la moitié supérieure du comblement (décapage 1), que dans la moitié inférieure (décapage 2), respectivement $65 \%$ et $35 \%$ de la masse du lot. Ils sont également éparpillés dans le dispositif de recouvrement.

Le loculus ayant été découvert complet, on peut considérer que l'on a retrouvé la quasi-totalité du lot qui y a été déposé. Il n'en va pas de même pour les os de la superstructure qui, elle, n'est que partiellement conservée. Le poids d'os déposés dans le loculus ne représente guère que $11,3 \%$ du poids moyen d'un squelette d'adulte brûlé, $1627,1 \mathrm{~g}$ selon McKinley (1993) et la part découverte dans le recouvrement n'en constitue que $6,2 \%$. 


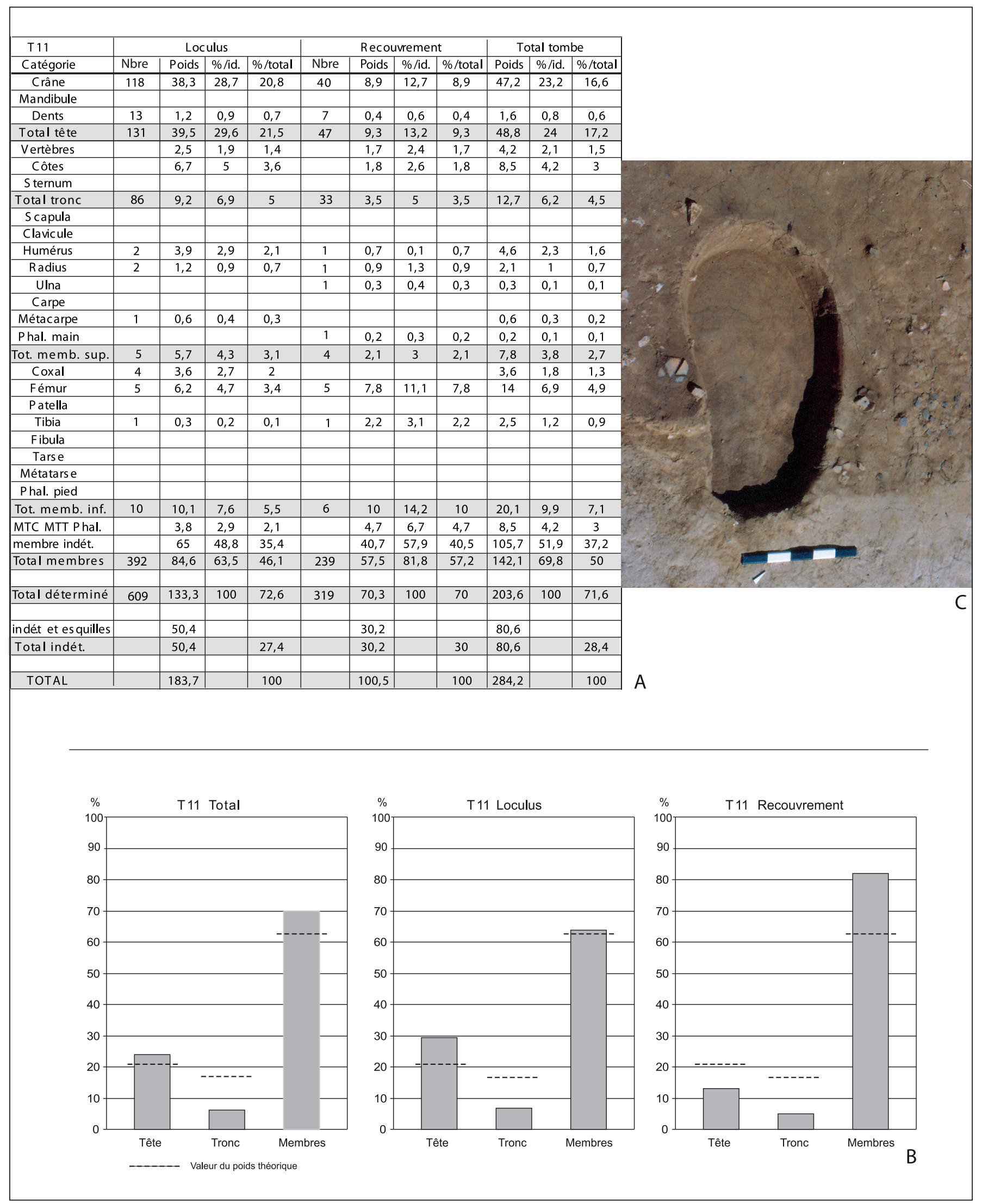

Fig. 14. Tombe T11. A : tableau de détermination des os humains (poids en grammes; calcul des \% sur la masse).

$\mathrm{B}$ : répartition du poids des os par grandes régions anatomiques (\% par rapport au total déterminé). C : vue du loculus en fin de fouille. 


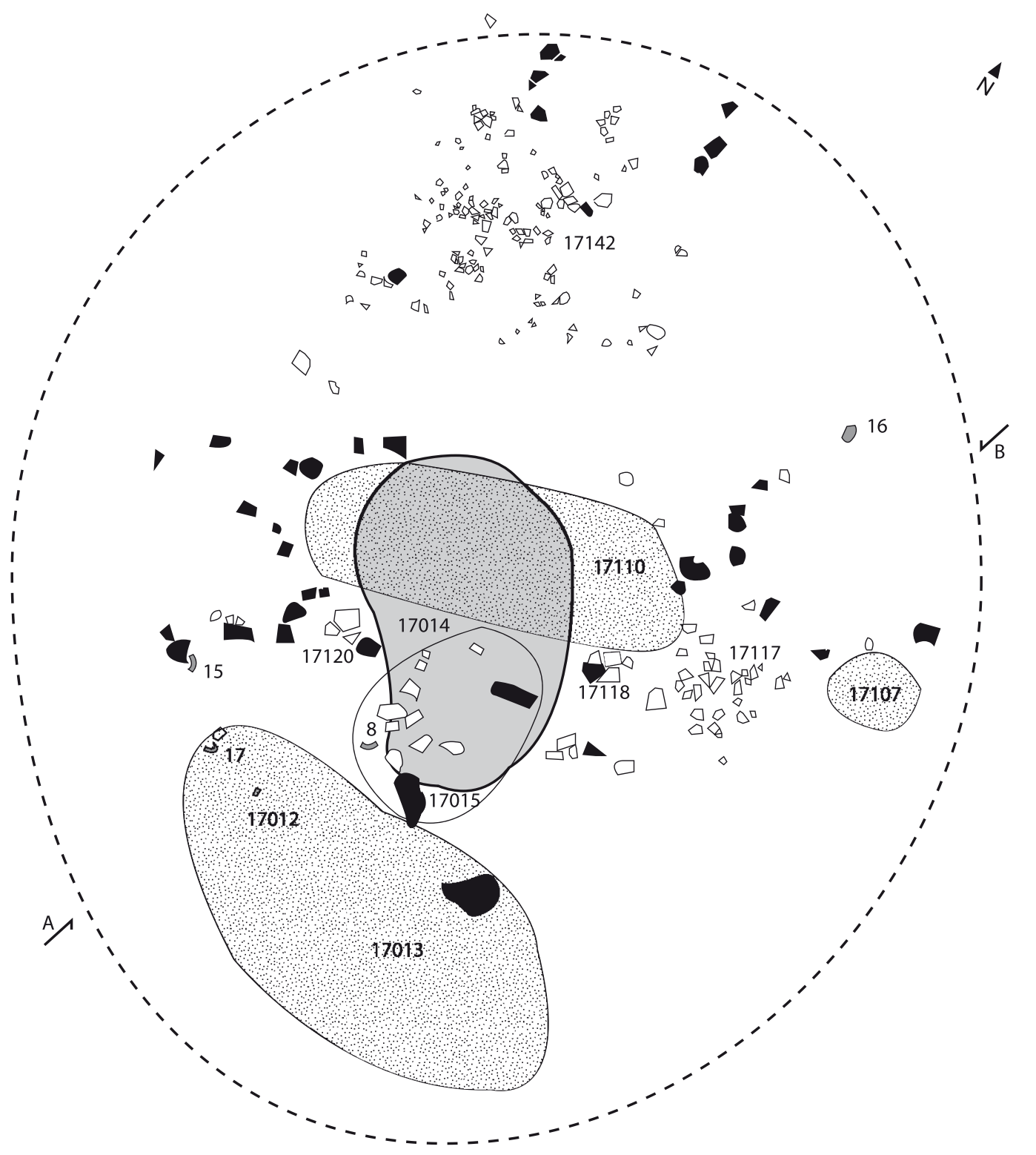

A
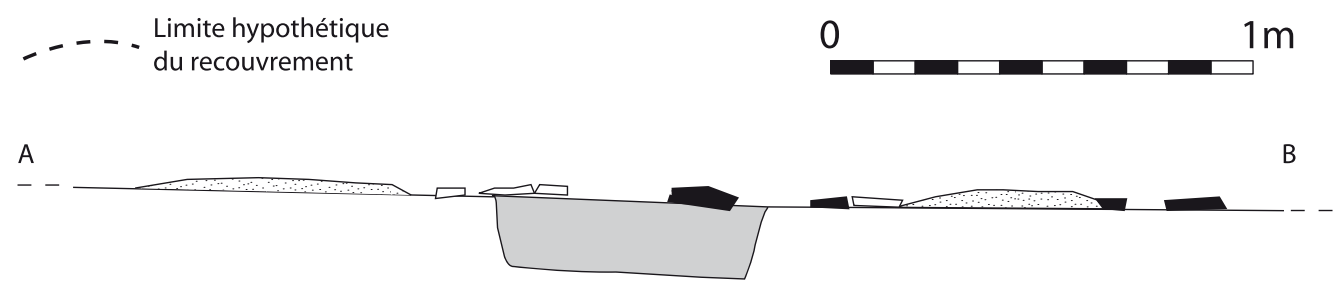

Loculus Lentille de sédiment avec os incinérés 
Les taux d'identification sont de 72,6\% du poids total des fragments pour le loculus et $70 \%$ pour la superstructure. Dans le loculus, avec 29,6\% du poids identifié, le crâne est sur-représenté (poids théorique : 20,4 \%). Cela se fait au détriment du tronc, 6,9\% au lieu des $17 \%$ attendus, car le taux des membres, $63,5 \%$, est conforme à la valeur théorique $(62,6 \%)$. Ces proportions diffèrent dans le lot provenant de la superstructure: le crâne est ici sous-représenté, $13,2 \%$ des fragments identifiés, au bénéfice des membres, très largement sur-représentés $(81,8 \%)$, tandis que le tronc, avec $5 \%$, est toujours aussi peu présent (fig. 14, A et B).

Le poids moyen des fragments déterminés, toutes régions anatomiques confondues, est le même pour le loculus et le dispositif de recouvrement et s'élève à $0,22 \mathrm{~g}$ (provenant du loculus : $0,30 \mathrm{~g}$ pour la tête, $0,11 \mathrm{~g}$ pour le tronc et $0,22 \mathrm{~g}$ pour les membres; provenant du recouvrement : $0,20 \mathrm{~g}$ pour la tête, $0,11 \mathrm{~g}$ pour le tronc et $0,24 \mathrm{~g}$ pour les membres). Pour les 7 fragments identifiés comme provenant des membres supérieurs, le poids moyen est de $1,12 \mathrm{~g}$; pour les 16 fragments identifiés comme provenant des membres inférieurs, il est de $1,18 \mathrm{~g}$.

\subsection{Le loculus}

Le loculus (US 17014) a un plan grossièrement ovalaire, de $1,1 \mathrm{~m}$ et $0,7 \mathrm{~m}$ d'axes (fig. 14, $\mathrm{C}$ et 15). Il est profond de $15 \mathrm{~cm}$; ses parois sont obliques, très abruptes, et son fond est plat. Son remplissage est formé d'une terre gris sombre, charbonneuse, dont la couleur tranche très nettement avec celle de l'encaissant. Outre les os humains, il renferme, dispersés en son sein, des morceaux d'objets incomplets, et exceptionnellement une pièce complète.

\section{Mobilier du loculus}

Objets personnels de parure et d'habillement :

- une fibule en bronze (fig. 16, $\mathrm{n}^{\circ} 1$ ), de type 8 ou 9 de C. Tendille, représentée par plusieurs morceaux : moitié de ressort bilatéral à six spires et corde externe (long. cons. : $13 \mathrm{~mm}$; diam. spire : $8 \mathrm{~mm}$ ), un petit morceau d'arc filiforme et plusieurs petits fragments déformés par le feu, non localisables ;

- une moitié de maillon de chaînette en bronze (fig. 16, $\mathrm{n}^{\circ} 2$ ); section circulaire ; diam. : $10 \mathrm{~mm}$. Cet objet a également brûlé.

Pièce métallique de fonction non déterminée:

- Pince en fer (fig. 16, $\mathrm{n}^{\circ}$ 7), formée d'une seule pièce repliée sur elle-même: d'un côté plaque sub-rectangulaire de profil incurvé, de l'autre, tige de section aplatie allant en s'amincissant depuis la plaque qu'elle prolonge ; cette tige forme une boucle en correspondance avec une moitié de la plaque; long.: $77 \mathrm{~mm}$; larg. plaque : $17 \mathrm{~mm}$; ép. plaque : 1,5 à $3 \mathrm{~mm}$; ép. tige : $3 \mathrm{~mm}$.

\section{Vaisselle céramique}

Le matériel céramique comprend une portion de vase et des tessons isolés d'environ 14 autres récipients :

- portion de la partie supérieure d'une urne non tournée de CNT-LOR U5d, variante d (13 tessons) : col tronconique rentrant, bord déversé formant méplat vers l'intérieur, lèvre aplatie, panse ovoïde peignée verticalement, épaule décorée d'un rang horizontal de petites impressions; diam. ouv. : $155 \mathrm{~mm}$; diam. max. : $180 \mathrm{~mm}$. Quatre autres tessons de ce vase proviennent des épandages recouvrant le loculus (US 17015 et 17110) et un autre, d'un des amas déposés sur le paléosol (US 17012-17013). Au total, ces 18 morceaux forment moins de $10 \%$ du vase (fig. 16, $\mathrm{n}^{\circ} 3$ );

-4 tessons d'urne non tournée de forme probable CNT-LOR U5d: panse ovoïde peignée verticalement (fig. 16, $n^{\circ} 4$ ) ;

-1 tesson d'urne non tournée de forme probable CNTLOR U5d : bord déversé, lèvre aplatie (fig. 16, $\mathrm{n}^{\circ} 5$ ) ;

-1 tesson de bord de vase non tourné (fig. 16, $\mathrm{n}^{\circ} 6$ ) ;

- 11 petits tessons de panses appartenant à autant d'urnes non tournées (non dessinés).

Os d'animaux (détermination A. Gardeisen) :

-2 fragments de porcs adultes (première phalange soudée de doigt latéral et extrémité distale d'humérus droit) ;

- 1 fragment d'extrémité distale de première phalange (cf. lapin);

- mandibule d'insectivore (cf. musaraigne);

- Indéterminés : 22 esquilles non brûlées.

\subsection{La structure de recouvrement}

De la structure de recouvrement subsistent deux sortes de vestiges (fig. 15) :

- un épandage d'objets posés horizontalement sur une partie du remplissage du loculus et débordant au sud-ouest sur le paléosol (US 17015), des portions de quatre vases écrasés en surface du paléosol de la nécropole, en périphérie du loculus (US 17117, 17118, 17120 et 17142), et quelques objets dispersés, aux alentours immédiats, également sur le paléosol (US 17104) ;

- des lentilles de terre limoneuse d'extension plus ou moins importante, de $6 \mathrm{~cm}$ d'épaisseur maximum, 
incluant du matériel, formant des restes d'une accumulation de matériaux qui devaient recouvrir le loculus et ses abords à la manière d'un petit tumulus très peu élevé (US 17012, 17013, 17107 et 17110).

Les limites de ce recouvrement ne sont pas connues, mais les restes conservés, lentilles et portions de vases, lui donnent au moins $3,3 \mathrm{~m}$ de diamètre ou de côté.

\section{Mobilier de la structure de recouvrement}

\section{L'épandage US 17015}

Sur la partie sud-ouest du comblement du loculus, débordant sur le paléosol, prend place une épandage d'objets disposés à plat, relativement groupés. Outre deux petites pierres plates calcaires $(130 \mathrm{~mm}$ sur $30 \mathrm{~mm}$ et $150 \mathrm{~mm}$ sur $8 \mathrm{~mm}$ ), non brûlées, figurent les pièces suivantes :

-3 morceaux d'un bracelet en bronze, soit $25 \%$ environ (fig. 16, $\mathrm{n}^{\circ} 8$ ); section en « $\mathrm{D} »$; pas de décor visible ; diam. int. : environ $60 \mathrm{~mm}$; haut. : $5 \mathrm{~mm}$; ép. : $3 \mathrm{~mm}$;

-3 tessons de l'urne non tournée $\mathrm{n}^{\circ} 3$ dont la majeure partie des morceaux prend place dans le remplissage du loculus ;

- 1 bord d'amphore massaliète de type A-MAS bd 9 (fig. 16, $n^{\circ} 9$ ) ;

- 1 petit tesson de bord de coupe à pâte claire massaliète (non dessiné) ;

-2 tessons d'une grande urne non tournée de forme probable CNT-LOR U5d ; panse ovoïde peignée horizontalement, épaule décorée d'impressions de peigne disposées en deux lignes brisées (fig. 16, $\mathrm{n}^{\circ} 10$ ) ;

- 6 tessons de panses de 6 vases non tournés (non dessinés) ;

- 1 morceau d'extrémité arrière de chenet modelé en terre cuite (fig. $\left.\mathbf{1 6}, \mathrm{n}^{\circ} 11\right)$.

Les portions de vases écrasés :

- Jouxtant le bord occidental du loculus (US 17120), 4 tessons jointifs de la panse arrondie convexe d'une grande jatte non tournée, soit moins de $5 \%$ du vase (non dessiné) ;

- À $10 \mathrm{~cm} \mathrm{du} \mathrm{bord} \mathrm{oriental} \mathrm{du} \mathrm{loculus} \mathrm{(US} \mathrm{17118),}$ 6 tessons jointifs d'une grande coupe non tournée de forme CNT-LOR C2d, représentant environ $15 \%$ du vase; panse arrondie convexe; bord rentrant à lèvre aplatie ; sous le bord, arrachement d'une anse horizontale en boudin ; diam. max. : $265 \mathrm{~mm}$ (fig. 16, $\mathrm{n}^{\circ} 12$ );

- Immédiatement à la suite de la précédente vers l'est (US 17117), 49 petits tessons, souvent jointifs, d'une urne non tournée de forme probable CNT-LOR U5d : départ de col tronconique rentrant, panse ovoïde à peignage horizontal, épaule décorée de longues impressions de peigne disposées en une ligne brisée; diam. max.: $240 \mathrm{~mm} .11$ autres petits tessons de ce vase prennent place à $1 \mathrm{~m}$ de là, dans la partie sud du recouvrement (17117). Au total ce sont $25 \%$ environ de cette urne qui sont représentés (fig. 16, $\mathrm{n}^{\circ} 13$ );

- A $30 \mathrm{~cm} \mathrm{du}$ bord septentrional du loculus (US 17142), 158 tessons, très souvent de très petite taille, parfois jointifs, d'une urne non tournée de forme CNT-LOR U5d, variante $\mathrm{d}$ : col tronconique rentrant, bord déversé avec méplat vers l'intérieur, lèvre aplatie, panse ovoïde peignée horizontalement, épaule décorée d'impressions au peigne disposées en deux lignes brisées, sur fond de peignage horizontal, fond plat avec, incisé sur la partie conservée, un trait large et peu profond, probable élément de croix ; diam. ouv. : $150 \mathrm{~mm}$; diam. max. : $175 \mathrm{~mm}$; haut. estimée : $210 \mathrm{~mm}$; environ $50 \%$ du vase est représenté (fig. 16, $\mathrm{n}^{\circ} 14$ ).

Les lentilles de terre :

Ces lentilles de limon semblable à celui qui recouvre la nécropole enferment quelques petits charbons de bois, des tessons et pierres calcaires centimétriques éclatées au feu, ainsi que de nombreux petits fragments d'os humains incinérés dispersés, 100,5 g au total. La lentille la plus vaste au sud (US 17012-17013) a livré $88 \mathrm{~g}$ de ces os ; au nord, celle qui couvre partiellement le loculus (US 17110), en contenait $8,5 \mathrm{~g}$; celle de l'est (US 17107), seulement 3,2 g.

Lentille US 17107 :

- 2 petits tessons d'amphore massaliète (non dessinés) ;

- portion de panse peignée et de col d'une urne non tournée, 13 tessons, soit moins de $10 \%$ du vase (non dessinés) ;

-4 petits tessons informes d'une urne ou d'une coupe non tournée, soit moins de $5 \%$ du vase (non dessinés) ;

- 9 petits tessons informes de 9 vases, urnes ou coupes (non dessinés).

Lentille US 17110 :

-2 tessons de l'urne non tournée $n^{\circ} 3$ dont la majeure partie des morceaux prend place dans le remplissage du loculus ;

- 3 tessons de l'urne non tournée $\mathrm{n}^{\circ} 13$ dont la majeure partie est écrasée sur le paléosol au nord-est (US 17117) ;

- 1 petit tesson de panse de vase en campanienne A ancienne (non dessiné) ;

- 1 tesson de panse d'urne ou d'œnochoé à pâte claire massaliète (non dessiné) ;

- 1 tesson d'urne non tournée de forme probable CNT-LOR U5d : bord évasé avec méplat vers l'intérieur, lèvre épaissie (fig. 16, $\left.\mathrm{n}^{\circ} 15\right)$. 


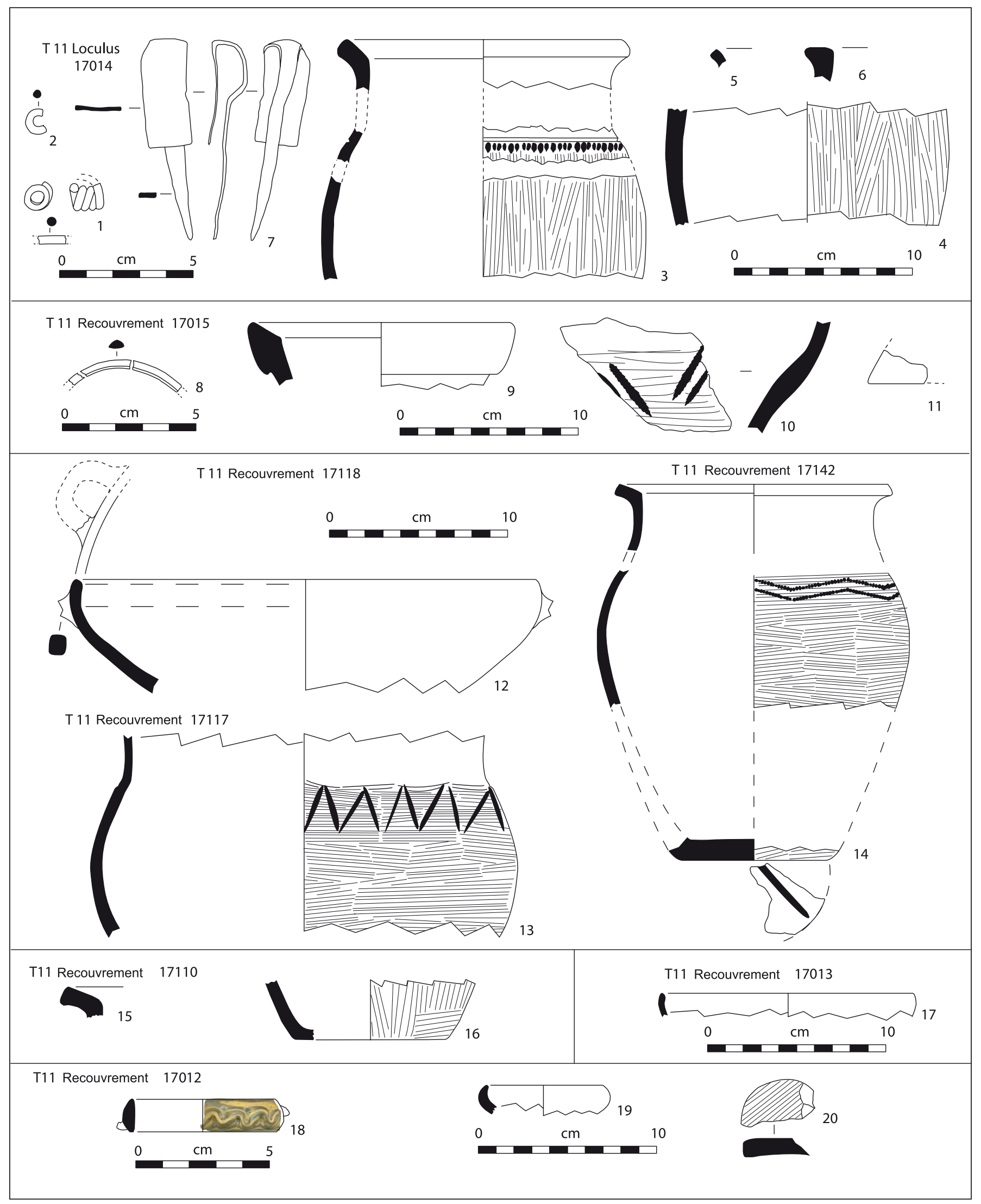

Fig. 16. Tombe T11. Mobilier (1, 2 et $8:$ bronze ; 3-6, 10, 12-16 et $20:$ céramique non tournée $; 7:$ fer ; 9 : amphore massaliète ; 11 : chenet modelé en terre cuite ; $18:$ verre $; 17$ et $19:$ céramique à vernis noir). 


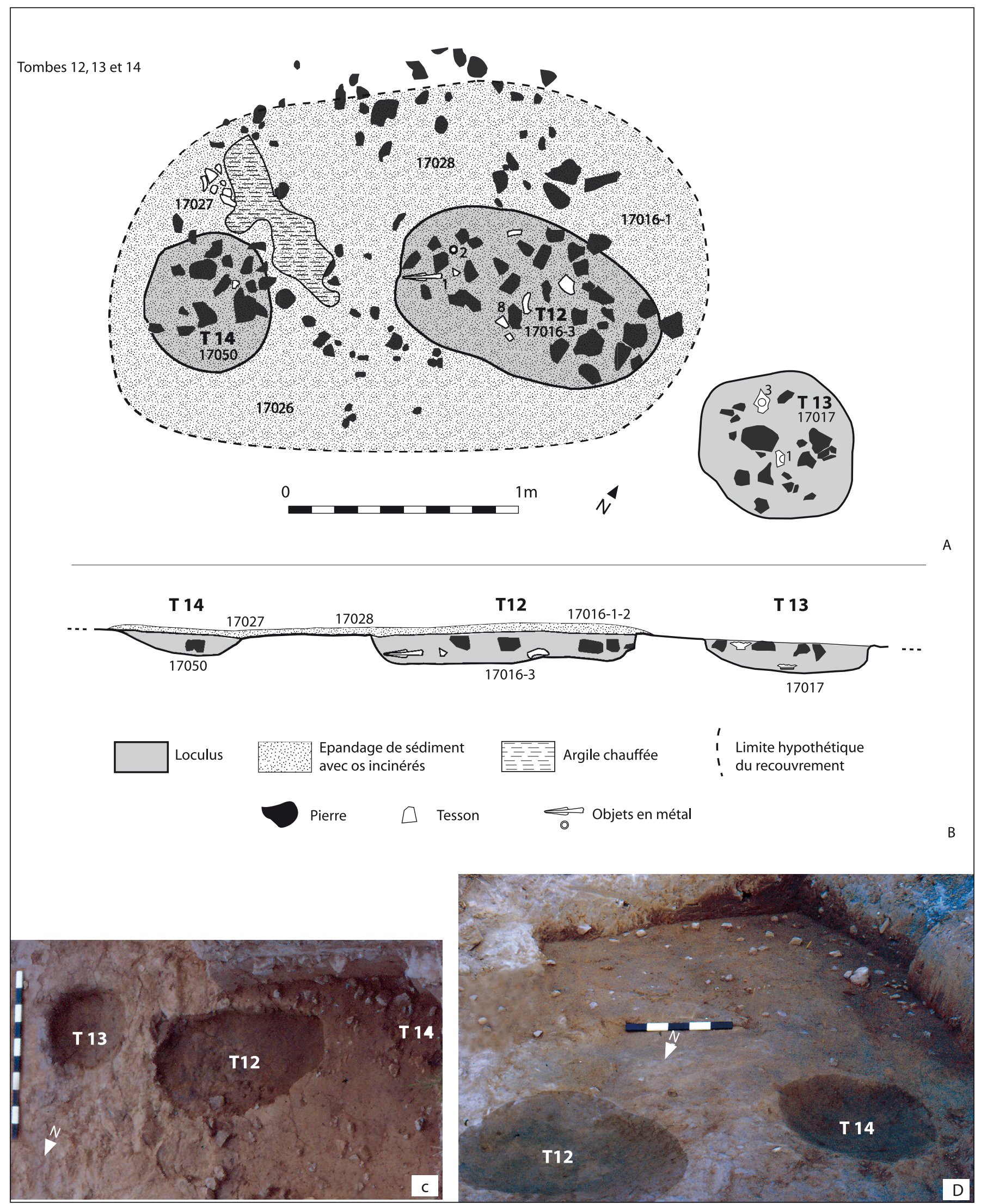

Fig. 17. Tombes $\mathrm{T} 12, \mathrm{~T} 13$ et T14. A : plan. B : coupe. C : vue prise du nord, loculus des tombes 12 et 13 en fin de fouille; comblement du loculus de la tombe 14. D : vue prise du nord, loculus des tombes 12 et 14 en fin de fouille. 
- 1 tesson d'urne non tournée : partie inférieure de panse peignée verticalement et obliquement, fond plat (fig. 16, $\left.\mathrm{n}^{\circ} 16\right)$;

- 38 petits tessons de panses peignées et de cols de plusieurs urnes non tournées (non dessinés).

\section{Lentille US 17012-17013 :}

- 1 morceau de bracelet en pâte de verre incolore; orné d'une onde en fort relief et de filets rapportés en verre jaune sur la tige et l'onde; section en « $\mathrm{D}$ » (groupe 4, forme 9 de Haevernick 1960) ; diam. int. : $55 \mathrm{~mm}$; haut. : $12 \mathrm{~mm}$; ép. : 4 à $6 \mathrm{~mm}$ (fig. 16, $\left.\mathrm{n}^{\circ} 18\right)$;

-1 tesson de l'urne non tournée $n^{\circ} 3$ dont la majeure partie des morceaux prend place dans le remplissage du loculus ;

- 1 tesson de bord de coupelle à vernis noir de l'atelier de Rosas, de forme Lamb. 27 (= Puig 11.b) ; diam. ouv. : $130 \mathrm{~mm}$ (fig. 16, $\mathrm{n}^{\circ} 17$ ). Il porte la trace d'un feu secondaire ;

- 1 tesson de bord de coupelle à vernis noir de l'atelier de Rosas, de forme Lamb. 24-25 (= Puig 2.a) ; diam. ouv. : $65 \mathrm{~mm}$ (fig. 16, $\mathrm{n}^{\circ} 19$ ). Ce tesson porte également la trace d'un feu secondaire ;

- 1 tesson de vase à vernis noir de l'atelier de Rosas, de forme indéterminée (non dessiné) ;

-7 petits tessons de panses de 7 vases non tournés (non dessinés) ;

- Moitié de rondelle découpée dans un tesson de panse peignée d'urne non tournée; diam.: $45 \mathrm{~mm}$; ép. $9 \mathrm{~mm}$ (fig. 16, $\left.\mathrm{n}^{\circ} 20\right)$;

- Petit morceau de base de chenet modelé en terre cuite (non dessiné) ;

- Plusieurs fragments de bronze en partie fondus (non dessinés).

Os d'animaux (détermination A. Gardeisen) :

- 12 fragments de caprinés (1 extrémité distale d'humérus gauche adulte , 1 extrémité distale de métapode adulte, 1 fragment dentaire adulte, 1 sésamoïde, 1 fragment de calcanéum droit, 1 bord latéral de diaphyse de fémur, 1 incisive très usée, 1 bord latéral de diaphyse d'os long (cf. radius), 1 extrémité distale de corps de côte (cf. capriné), 1 fragment sous-articulaire de corps de côte juvénile (cf. capriné), 1 fragment de neurocrâne (cf. capriné), 1 fragment sous-articulaire de corps de côte juvénile (porc ou capriné ?) ;

- 1 fragment de porc juvénile (extrémité proximale non soudée de deuxième phalange) ;

- 1 fragment de corps de côte d'un animal de grande taille (cf. bœuf) ;

- 1 fragment de canidé (diaphyse d'ulna);

- 1 fragment d'extrémité proximale de fibula d'oiseau (cf. galliforme) ;
- Indéterminés : 54 esquilles non brûlées, 6 esquilles brûlées blanches et 5 esquilles brûlées bleu-noir.

\section{La tombe $\mathrm{T} 12$}

\section{1. État et composantes de la tombe}

La tombe 12 est celle d'un adulte, âgé probablement de plus de trente ans. Le sexe n'est pas déterminable, mais le mobilier laisse apparaitre une connotation masculine. Cette sépulture comprend un loculus et une structure de surface qui recouvre aussi la tombe 14 , dont le loculus est tout proche, à seulement $0,5 \mathrm{~m}$ de celui de la tombe 12 . L'ensemble a pu être entièrement fouillé (fig. 17).

\subsection{Le défunt}

Le défunt est représenté par, au moins, 742 g d'os brûlés (fig. 18, A). Il s'agit là du lot recueilli dans le loculus (US 17016-2 et 17016-3) ; mais ce n'est sans doute pas la totalité des restes de ce défunt déposés dans cette sépulture, car le recouvrement commun aux tombes 12 et 14 a aussi livré 561,4 g d'os incinérés qu'il est impossible de répartir entre les deux adultes de ces deux sépultures.

La morphologie des os et la fermeture des apex des dents, y compris sur une troisième molaire, indiquent un adulte. Ce pourrait être un adulte âgé de plus de trente ans, mais pas un vieillard, car, si les sutures sont encore libres sur la face exocrânienne, elles sont en cours de synostose sur l'endocrâne. Le sexe anthropologique ne peut pas être précisé.

Le poids d'os déposés dans le loculus représente 45,6\% du poids moyen d'un squelette d'adulte brûlé (1627,1 g selon McKinley 1993). Les taux d'identification sont de $89,2 \%$ du poids total des fragments. La sur-représentation des membres est importante, 74,5\% du poids identifié pour un poids théorique de $62,6 \%$; cela se fait au détriment du crâne, $16,6 \%$ du poids identifié au lieu des 20,4\% attendus, et surtout du tronc, nettement sous-représenté, $8,9 \%$ (poids théorique : $17 \%$ ) (fig. 18, B). La couleur blanc crayeux révèle une température de crémation supérieure à $650^{\circ}$ selon le barème de E. Bonnucci et G. Grazziani (1975), et les morceaux de diaphyses sont très souvent fissurés en ondes concentriques, indice d'une incinération d'os frais. 


\begin{tabular}{|c|c|c|c|c|c|c|c|c|c|c|}
\hline \multirow{2}{*}{$\begin{array}{l}\text { T } 12 \\
\text { Catégorie }\end{array}$} & \multicolumn{3}{|c|}{ Loculus $17016-2$} & \multicolumn{3}{|c|}{ Loculus 17016-3 } & \multicolumn{4}{|c|}{ Total loculus } \\
\hline & Poids & $\% /$ id. & $\% /$ total & Poids & $\% /$ id. & $\% /$ total & Nbre & Poids & $\% /$ id. & $\% /$ total \\
\hline Crâne & 4,8 & 28,9 & 21,3 & 94,2 & 14,6 & 13,1 & 254 & 99,1 & 15 & 13,3 \\
\hline Mandibule & & & & 3,9 & 0,6 & 0,5 & 5 & 3,9 & 0,6 & 0,5 \\
\hline Dents & 0,2 & 1,2 & 0,8 & 8,3 & 1,3 & 1,2 & 75 & 8,5 & 1,3 & 1,1 \\
\hline Total tête & 5 & 30,1 & 22,2 & 106,4 & 16,5 & 14,8 & 334 & 111,3 & 16,8 & 14,9 \\
\hline Vertèbres & 0,3 & 1,8 & 1,3 & 35,6 & 5,5 & 4,9 & & 36 & 5,4 & 4,8 \\
\hline Côtes & 0,5 & 3 & 2,2 & 22,3 & 3,5 & 3,1 & & 22,8 & 3,4 & 3,1 \\
\hline \multicolumn{11}{|l|}{ S ternum } \\
\hline Total tronc & 0,8 & 4,8 & 3,6 & 58 & 9 & 8,1 & 467 & 58,8 & 8,9 & 7,9 \\
\hline S capula & & & & 1,4 & 0,2 & 0,2 & 9 & 1,4 & 0,2 & 0,2 \\
\hline \multicolumn{11}{|l|}{ Clavicule } \\
\hline Humérus & 0,6 & 3,6 & 2,7 & 6,1 & 0,9 & 0,8 & 6 & 6,7 & 1 & 0,9 \\
\hline Radius & & & & 19,3 & 3 & 2,7 & 16 & 19,3 & 2,9 & 2,6 \\
\hline Ulna & & & & 9,9 & 1,5 & 1,4 & 5 & 9,9 & 1,5 & 1,3 \\
\hline Carpe & & & & 0,6 & 0,1 & 0,1 & 3 & 0,6 & 0,1 & 0,1 \\
\hline \multicolumn{11}{|l|}{ Métacarpe } \\
\hline \multicolumn{11}{|l|}{ Phal. main } \\
\hline Tot. memb. sup. & 0,6 & & & 37,3 & 5,8 & 5,2 & 39 & 37,9 & 5,7 & 5,1 \\
\hline Coxal & & & & 3,5 & 0,5 & 0,5 & 4 & 3,5 & 0,5 & 0,5 \\
\hline Fémur & & & & 7,8 & 1,2 & 1,1 & 4 & 7,8 & 1,2 & 1,1 \\
\hline \multicolumn{11}{|l|}{ Patella } \\
\hline Tibia & 2,3 & 13,6 & 10,2 & 27,8 & 4,3 & 3,9 & 20 & 30 & 4,5 & 4 \\
\hline Fibula & & & & 1,2 & 0,2 & 0,2 & 2 & 1,2 & 0,2 & 0,2 \\
\hline Tarse & & & & 12,9 & 2 & 1,8 & 5 & 12,9 & 1,9 & 1,7 \\
\hline \multicolumn{11}{|l|}{ Métatarse } \\
\hline Phal. pied & & & & 0,8 & 0,1 & 0,1 & 2 & 0,8 & 0,1 & 0,1 \\
\hline Tot. memb. inf. & 2,3 & & & 54,1 & 8,4 & 7,5 & 37 & 56,3 & 8,5 & 7,6 \\
\hline MTC MTT P hal. & 1,6 & 9,6 & 7,1 & 27,5 & 4,3 & 3,8 & & 29,2 & 4,4 & 3,9 \\
\hline membre indét. & 6,3 & 38 & 28 & 362 & 56,1 & 50,3 & & 368,3 & 55,6 & 49,6 \\
\hline Total membres & 10,8 & 65 & 48 & 480,9 & 74,5 & 66,8 & 2244 & 491,7 & 74,3 & 66,3 \\
\hline Total déterminé & 16,6 & 100 & 73,8 & 645,3 & 100 & 89,7 & 3045 & 661,9 & 100 & 89,2 \\
\hline indét. et es quilles & 5,9 & & & 74,3 & & & & 80,1 & & 10,8 \\
\hline Total indét. & 5,9 & & 26,2 & 74,3 & & 10,3 & & 80,1 & & 10,8 \\
\hline TOTAL & 22,5 & & 100 & 719,5 & & 100 & & 742 & & 100 \\
\hline
\end{tabular}
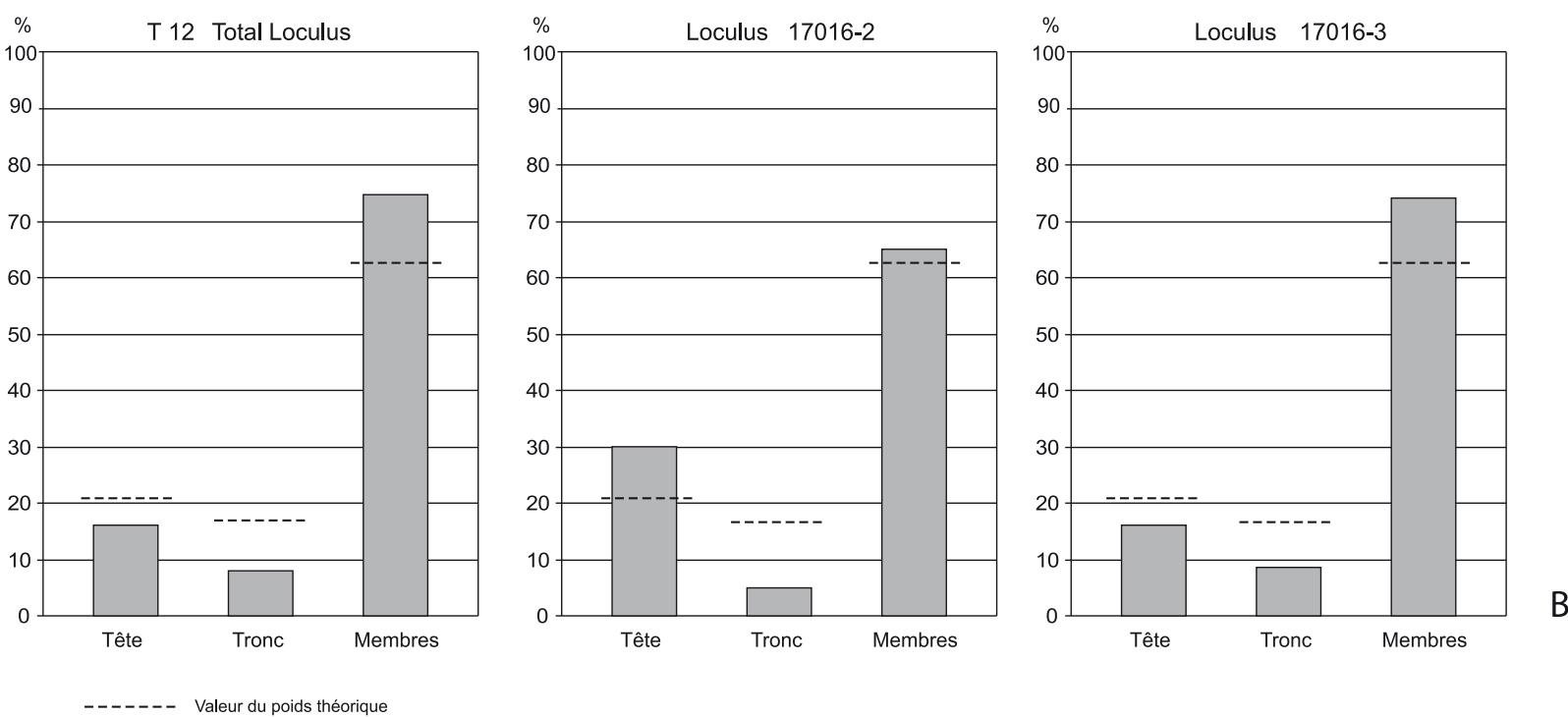

Fig. 18. Tombe T12. A : tableau de détermination des os humains (poids en grammes; calcul des \% sur la masse). B : répartition du poids des os par grandes régions anatomiques (\% par rapport au total déterminé). 
Les os ont été placés, dans le loculus, hors de tout contenant, mais selon deux procédés. La grande majorité, $719,5 \mathrm{~g}$ soit $97 \%$, parfois de grande taille et souvent amalgamés entre eux, provient d'un amoncellement de magma cendreux et charbonneux, gris foncé à noirâtre, occupant la partie centrale de la fosse (US 17016-3). Les autres vestiges, plus fragmentés et moins nombreux $(22,5 \mathrm{~g})$, sont mélangés à la terre du comblement, en sa partie supérieure (US 17016-2). Aucune incompatibilité n'existe entre les deux lots qui, par ailleurs, proviennent des mêmes régions du corps.

Le poids moyen des fragments déterminés, toutes régions anatomiques confondues, est de $0,22 \mathrm{~g}(0,33 \mathrm{~g}$ pour la tête, $0,16 \mathrm{~g}$ pour le tronc et $0,22 \mathrm{~g}$ pour les membres). Pour les 39 fragments identifiés comme provenant des membres supérieurs, le poids moyen est de $0,97 \mathrm{~g}$; pour les 37 fragments identifiés comme provenant des membres inférieurs, il est de 1,52 g.

\subsection{Le loculus}

Le loculus (US 17016-2 et 3) a un plan ovalaire très allongé orienté SO - NE, de 1,30 m sur 0,65 m d'axes. Sa profondeur maximum est de $0,15 \mathrm{~m}$ vers le centre. Ses parois sont abruptes, proches de la verticale, et son fond est à peu près plat. La partie supérieure du remplissage du loculus (US 17016-2) est formée d'une terre grise très sombre incluant de nombreux petits fragments de charbons de bois ainsi que des morceaux d'os humains incinérés. En son sommet se trouvent une trentaine de petites pierres calcaires non roulées, de 4 à $18 \mathrm{~cm}$ d'arête, deux morceaux jointifs d'une table de meule en basalte de 7 à $8 \mathrm{~cm}$ d'arête, et un tesson de dolium, de même calibre. Deux de ces pierres ont éclaté, mais leurs morceaux ont été retrouvés dans le loculus. La partie médiane et la base du remplissage (US 17016-3) est formé de cendres blanchâtres enfermant des amas de magma d'os incinérés.

Le mobilier est dispersé dans la partie basse du remplissage au sein des amas de cendres: une pointe de lance, deux éléments de suspension d'épée, un maillon de chaînette et trois morceaux d'objets non déterminés, trente-six tessons se rapportant à une ou deux amphores massaliètes et seize pièces de vaisselle. Certains de ces objets montrent des traces d'un feu secondaire. On soulignera que des morceaux de la même coupe à vernis noir (fig. 19, $n^{\circ} 8$ ) se rencontrent dans ce loculus et dans la structure de recouvrement, et que des tessons de la même coupe claire massaliète (fig. $19, \mathrm{n}^{\circ} 13$ ) prennent place dans ce loculus, dans la structure de recouvrement, mais aussi dans le loculus de la tombe 13 .

\section{Mobilier du loculus}

Objets personnels, armement et fourniment :

- Pointe de lance en fer presque complète (il manque les deux extrémités) ${ }^{\mathbf{1 0}}$; lame triangulaire à carène très basse et tranchants rectilignes, nervure axiale très saillante de section losangique et longue douille conique avec nervure de l'empennage se prolongeant sur presque toute sa longueur tout en s'affaiblissant progressivement. Long. tot. cons. : $171 \mathrm{~mm}$; long. tot. restituée: $180 \mathrm{~mm}$; long. lame restituée : $115 \mathrm{~mm}$; long. partie pénétrante : $110 \mathrm{~mm}$; larg. lame : 32,9 mm ; long. douille restituée : $65 \mathrm{~mm}$; diam. douille : $21,7 \mathrm{~mm}$; part de la lame : $67 \%$; effilement de la lame : $27 \%$; portion pénétrante de la lame : $96 \%$. Cet objet n'a pas subi de déformation (fig. 19, $\mathrm{n}^{\circ} 1$ et fig. 115).

- Deux éléments de la ceinture de suspension d'un fourreau d'épée :

- anneau circulaire en fer (fig. 19, $\mathrm{n}^{\circ} 2$ ), de section circulaire ; diam. : $34 \mathrm{~mm}$; haut. : 4,5 à $5,5 \mathrm{~mm}$;

- agrafe de ceinture, en fer (fig. 19, $\mathrm{n}^{\circ} 3$ et fig. 115); boucle rectangulaire; corps cordiforme; crochet terminé par un bouton relevé, en calotte sphérique ; long. : $48 \mathrm{~mm}$; larg. : $20 \mathrm{~mm}$;

- Morceau d'un maillon de chaînette en bronze (fig. 19, $\mathrm{n}^{\circ} 4$ ) ; section ovalaire ; diam. : $10 \mathrm{~mm}$. Cet objet a subi l'action d'un feu secondaire.

Objets métalliques indéterminés :

- Morceau d'un anneau de serrage en bronze (fig. 19, $\mathrm{n}^{\circ}$ 5) ; section en lunule ; diam. int. : $60 \mathrm{~mm}$. ; objet indéterminé. Cet objet a subi l'action d'un feu secondaire ;

- Petit morceau de tôle de bronze coulée (fig. 19, $\mathrm{n}^{\circ} 6$ ), déformé par l'action d'un feu secondaire ; ép. : $1 \mathrm{~mm}$; objet indéterminé ;

- Segment de tige en fer (fig. 19, $\mathrm{n}^{\circ} 7$ ), rectiligne, de section circulaire ; diam. : 6 à $8 \mathrm{~mm}$; long. cons. : $20 \mathrm{~mm}$.

\section{Matériel céramique}

Le mobilier céramique comprend un vase déposé sans doute complet dans le loculus, un autre, sans doute complet, dont les morceaux ont été répartis dans le loculus et le recouvrement de cette tombe ainsi que dans le loculus de la tombe 13 :

- 1 coupe à vernis noir de l'atelier de Rosas, de forme Lamb. 28a-b (= forme Puig 13.a), presque complète (fig. 19, $\mathrm{n}^{\circ} 8$ ) ; diam. $\max$. : $105 \mathrm{~mm}$; haut. : $42 \mathrm{~mm}$;

10 La terminologie utilisée ici, les mesures et les rapports pris en compte sont ceux qui ont été mis au point dans Dedet, Marchand, à paraître). 


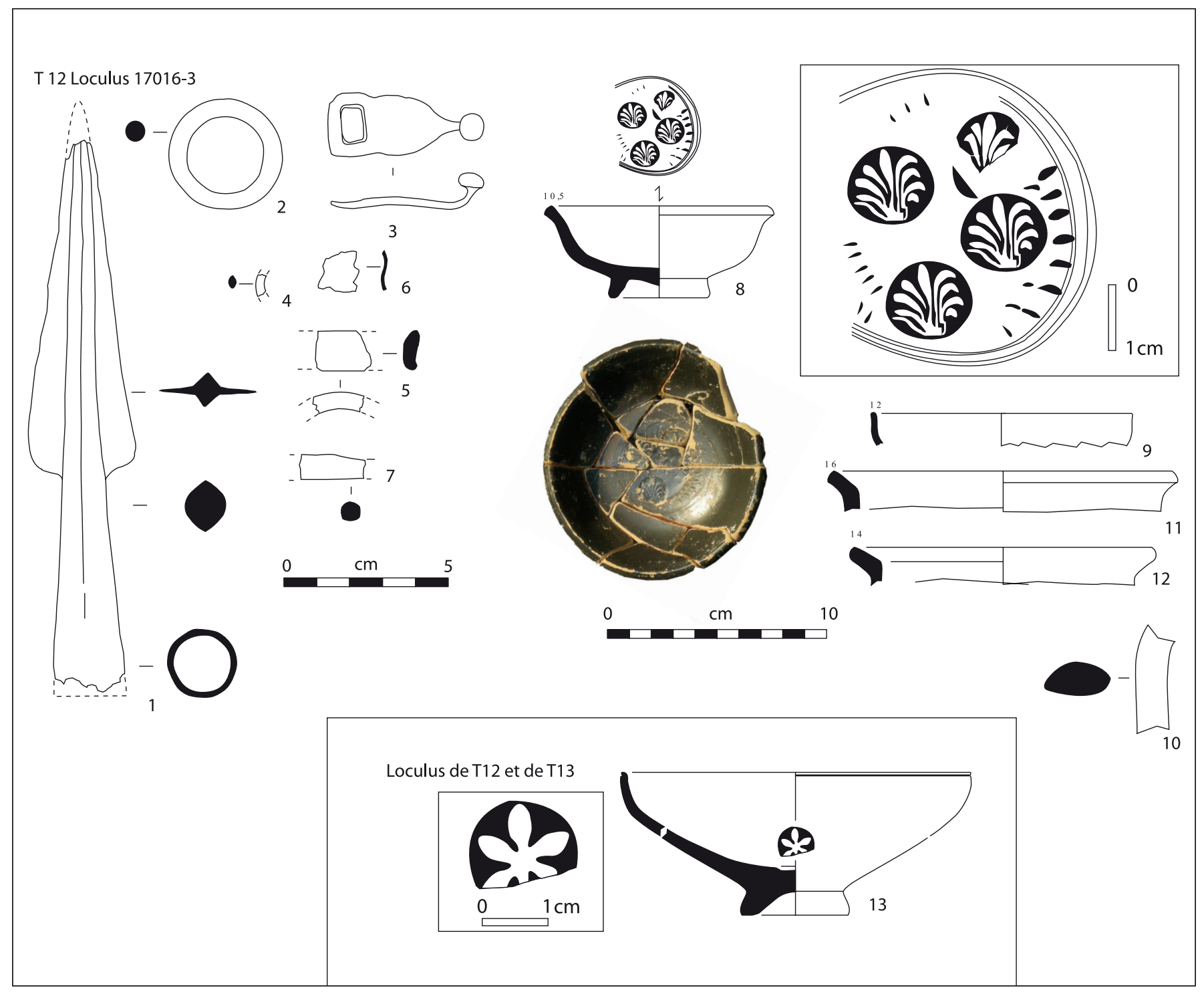

Fig. 19. Tombes T12 et T13. Mobilier (1-3 et $7:$ fer $; 4-6:$ bronze $; 8:$ céramique à vernis noir $; 9:$ céramique grise peinte ; $10:$ amphore massaliète ; 11 et 12 : céramique non tournée ; $13:$ céramique à pâte claire).

diam. base : $45 \mathrm{~mm}$; sur le fond, quatre palmettes parallèles entourées d'un cercle de guillochis. La grande majorité des tessons de ce vase sont dans le loculus, mais deux autres morceaux proviennent de la couverture de la tombe (US 17028);

- 10 tessons d'un bol à pâte claire massaliète de forme CL-MAS 321 proche de la forme Lamb. 27a-b ; panse arrondie ; bord légèrement rentrant, souligné par une rainure horizontale à l'extérieur; lèvre arrondie et amincie; pâte de couleur rosée ; au centre du fond, rosette à six pétales en relief dans un cartouche circulaire de $16 \mathrm{~mm}$ de diamètre, décor bien attesté sur cette forme de coupe à Olbia (Bats 1988, p. 179); surfaces couvertes d'une peinture brun rouge dont ne subsistent que quelques plages adhérant mal à l'épiderme à l'extérieur ; diam. ouv. : 160 mm. Deux tessons de ce vase proviennent du loculus et trois autres de la couverture (US 17028), d'autres morceaux du bord et toute la partie inférieure de ce vase figurent dans le loculus de la tombe 13 (fig. 19, $\mathrm{n}^{\circ} 13$ ) ;

- 1 tesson de bord de coupe grise peinte de forme GR-PEINTE 4 (Py et al. 2001, p. 1121); panse légèrement ondulée; bord légèrement dégagé et lèvre arrondie ; diam. ouv. : $120 \mathrm{~mm}$ (fig. 19, $\mathrm{n}^{\circ}$ 9) ;

- 1 éclat de vase à pâte claire massaliète (non dessiné) ;

- 2 petits tessons jointifs d'une panse d'amphore massaliète et un morceau d'anse de petite taille (fig. 19, $\mathrm{n}^{\circ} 10$ ), appartenant peut-être au même exemplaire ; le morceau d'anse porte la trace d'un feu secondaire ; 
- 14 petits tessons, appartenant probablement à 7 urnes non tournées :

- 3 tessons d'une grande urne de forme probable CNT-LOR U5d : bord déversé, lèvre aplatie ; panse peignée ; diam. ouv. 160 m (fig. 19, $\mathrm{n}^{\circ} 11$ );

- 1 bord et 1 tesson d'urne non tournée de forme probable CNT-LOR U5d : col tronconique rentrant, bord déversé avec méplat vers l'intérieur, lèvre arrondie ; diam. ouv. $140 \mathrm{~mm}$ (fig. 19, $\mathrm{n}^{\circ} 12$ ) ;

- 2 tessons jointifs d'un col d'urne, avec une très nette différence de couleur de part et d'autre de la cassure montrant l'effet d'un feu secondaire (non dessiné) ;

- 2 tessons d'un autre col d'urne (non dessiné) ;

- 5 tessons de 3 panses peignées d'urnes (non dessiné) ;

- 1 tesson de dolium indigène portant un cordon horizontal lisse et deux éclats (non dessiné).

Os d'animaux (détermination A. Gardeisen)

Le lot comprend les vestiges de deux dépôts différenciés et bien caractérisés ainsi que des restes isolés :

- 66 restes fragmentés et carbonisés d'un crâne de porcelet d'environ 3 mois (4 fragments du neurocrâne, 9 des deux mandibules, 31 de dents lactéales isolées et des esquilles osseuses présentant le même stade de carbonisation);

- 27 fragments du gril costal d'un capriné juvénile, carbonisés à blanc, provenant de la même série des cinq premières côtes (au moins), droites ;

-2 fragments de caprinés (de racine de dent jugale et de coxal) ;

- esquille d'os compact épais présentant des négatifs d'enlèvements corticaux d'un animal de grande taille ;

- 391 esquilles brûlées à des divers degrés, de la couleur noire à la couleur blanche, qui proviennent potentiellement en partie des restes déterminés comme dépôts initiaux (crâne de porcelet et gril costal de capriné) mais aussi de divers os longs appartenant à des animaux matures ;

- Indéterminés : 4 esquilles non brûlées.

\section{La tombe T13}

\section{1. État et composantes de la tombe}

La tombe T13 abrite un seul défunt incinéré, un jeune enfant, âgé de trois à cinq ans. La quasi totalité de son matériel provient du loculus, qui a été entièrement fouillé. La structure de recouvrement a presque entièrement disparu. Les rares vestiges découverts reposent sur le paléosol, immédiatement au N-NE, montrant que la partie supérieure du loculus n'a pas été tronquée (fig. 17, A, B et C).

\subsection{Le défunt}

Ce jeune enfant est représenté par 71,5 g d'os brûlés. Ces derniers proviennent presque tous $(69,8 \mathrm{~g}) \mathrm{du}$ loculus (US 17017); les rares esquilles issues des restes de la superstructure ont un poids négligeable $(1,7 \mathrm{~g})$. La détermination de l'âge, entre trois et cinq ans, repose sur un faisceau d'éléments dont la précision est variable : aucun fragment de calotte crânienne n'est épais ; les morceaux de diaphyses offrent un aspect général très gracile; une seconde phalange du pied montre une surface métaphysaire, de même que deux autres morceaux d'extrémités proximales de phalanges de pied ou de main; de nombreux germes de dents définitives indiquent un âge de 4 ans plus ou moins douze mois, en particulier les germes de $\mathrm{M}^{1}, \mathrm{C}^{\prime} \mathrm{d}, \mathrm{C}$,g et les fragments de germes de trois molaires et de deux incisives.

Les pièces osseuses sont très fragmentées. L'incinération a affecté des os frais, comme le montrent les fissurations en ondes concentriques des diaphyses. Presque tous les restes ont une couleur blanc crayeux, indiquant une température de crémation de l'ordre de $650^{\circ}$ selon le barème de E. Bonnucci et G. Grazziani (Bonucci, Grazziani 1975).

Toutes les parties du squelette ont été concernées par le prélèvement sur le bûcher (fig. 20, A). L'indice pondéral crânien s'établit à $50,4 \%$, celui du tronc à $5,2 \%$ et celui des membres à 44,4\% ; mais un tiers des fragments n'a pu être déterminé (fig. 20, B). La prééminence du crâne, très nettement en dessus de la valeur théorique de $20,4 \%$ pour les adultes selon Lowrance et Latimer (dans Krogman 1978), tient sans doute au fait que la tête est en proportion plus grosse chez les jeunes enfants (Trotter, Hixon 1974, cité dans Lenorzer 2009, p. 88).

Les restes osseux sont dispersés dans l'ensemble du comblement du loculus, hors de tout contenant, et aucune disposition particulière n'a été perçue. En particulier, aucune différence importante de répartition des pièces osseuses n'apparaît entre les cinq décapages effectués.

Le poids moyen des fragments déterminés, toutes régions anatomiques confondues, est de $0,09 \mathrm{~g}(0,12 \mathrm{~g}$ pour la tête, $0,06 \mathrm{~g}$ pour le tronc et $0,08 \mathrm{~g}$ pour les membres). 


\subsection{Le loculus}

Le loculus (US 17018) a un plan grossièrement ovalaire et mesure $75 \mathrm{~cm}$ sur son grand axe E-O et $65 \mathrm{~cm}$ sur son petit axe N-S. Sa profondeur maximale est de $15 \mathrm{~cm}$ vers le centre. Ses parois sont obliques, plus abruptes vers l'Est et le Sud, et son fond est légèrement arrondi (fig. 17, A, B et C).

Le remplissage (US 17017) est formé d'une terre gris sombre gluante, très charbonneuse, dont la couleur tranche très nettement avec celle de l'encaissant.

Il a été fouillé en cinq décapages théoriques de $3 \mathrm{~cm}$ d'épaisseur. Dans toute son épaisseur il renferme de très nombreux petits fragments de charbons de bois et de très nombreux morceaux d'os humains incinérés. Au sommet du remplissage se trouve la partie inférieure d'un bol à pâte claire massaliète qui a subi l'action du feu (fig. 19, $\mathrm{n}^{\circ} 13$ ) et dont des tessons de la partie supérieure gisent également dans le loculus et dans la structure de recouvrement de la tombe T12. Vingt-quatre pierres calcaires non roulées, de 2 à $12 \mathrm{~cm}$ d'arête, prennent place dans la moitié supérieure du remplissage (décapages 1 à 3). Certaines ont subi l'action du feu. Dispersés dans le reste du comblement figurent six petits tessons non tournés se rapportant à autant de récipients, et un fragment de bord de la coupe claire massaliète déjà mentionnée (fig. 19, $\mathrm{n}^{\circ} 13$ ), montrant nettement les traces d'un feu secondaire. Sur le fond du loculus git un fond de coupelle à vernis noir (fig. 20, C, $\mathrm{n}^{\circ} 1$ ).

\section{Mobilier du loculus}

Le matériel du loculus comprend les tessons de deux coupes dont d'autres morceaux ont été découverts dans le recouvrement de la tombe ou dans la tombe T12, ainsi que des tessons isolés de six autres récipients :

- 1 tesson de bord, portant des traces de feu secondaire, et plusieurs morceaux du fond de la coupe à pâte claire massaliète CL-MAS 321 dont plusieurs tessons ont été déposés dans le loculus et le recouvrement de la tombe T12 (fig. 19, $\mathrm{n}^{\circ} 13$ );

- 1 tesson de fond de bol CAMP-A 27a-b, en campanienne A ancienne, portant en son centre, dans un cartouche circulaire de $12 \mathrm{~mm}$ de diamètre, une rosette à six pétales en forme de larmes au dessin très fin, dont seul le contour est en relief; diam. fond : $60 \mathrm{~mm}$ (fig. 20, $\mathrm{C}, \mathrm{n}^{\circ} 1$ ). Il a subi l'action d'un feu secondaire. Trois autres morceaux du même vase, dont deux se raccordent à ce fond, se trouvaient sur le sol, à proximité de ce loculus, à la base du dispositif de recouvrement de la tombe (voir infra) ;
- 1 tesson d'urne non tournée de forme probable CNT-LOR U5d : bord déversé à lèvre arrondie ; diam. ouv. : $150 \mathrm{~mm}$ (fig. 20, C, $\mathrm{n}^{\circ} 2$ ) ;

- 1 tesson d'urne non tournée : bord déversé à lèvre arrondie (fig. 20, C, $\mathrm{n}^{\circ} 3$ ) ;

-3 tessons de panses de trois autres urnes non tournées (non dessinés) ;

- 1 tesson de couvercle non tourné de forme CNTLOR V2a : bord dans la continuité de la paroi rectiligne, lèvre aplatie (fig. 20, $\mathrm{C}, \mathrm{n}^{\circ} 4$ ) ;

Os d'animaux (détermination A. Gardeisen) :

- 1 fragment de dent jugale de capriné, brûlée ;

-1 esquille d'os long d'animal de petite taille, brûlée ;

- Microfaune : 1 fragment de côte ;

- Malacofaune : petit lot d'escargots.

- Poisson (détermination G. Piquès) : 2 vertèbres de corb (cf. Sciaena umbra Linné, 1758), 2/3e caudale et $7^{\mathrm{e}}$ caudale d'un même individu; la dernière marque de croissance situe la saison de mort du poisson vers le début de l'automne.

\subsection{La structure de recouvrement}

Quelques rares os incinérés et trois morceaux de la panse du vase à vernis noir, dont le fond a été placé dans le loculus (fig. 20, $\mathrm{C}, \mathrm{n}^{\circ} 1$ ), sont dispersés sur le sol au nord-est du loculus de la tombe T13, à environ $1 \mathrm{~m}$ de ce dernier. Ces tessons ont fortement brûlé et ont éclaté à la chaleur. Ce matériel parait provenir du démantèlement d'un amoncellement de terre (US 17007) recouvrant ce loculus.

\section{La tombe T14}

\section{1. État et composantes de la tombe}

La tombe T14 a accueilli un adulte de sexe non déterminé, plus jeune et gracile que celui de la tombe T12 voisine à laquelle l'associe la même structure de recouvrement. La différence des poids moyens des fragments osseux déterminés du lot provenant de ce loculus avec celui de la tombe T12 montre qu'il s'agit bien de deux incinérations distinctes et donc de deux sujets différents. Là aussi, d'après le mobilier déposé, il s'agirait d'un homme. 


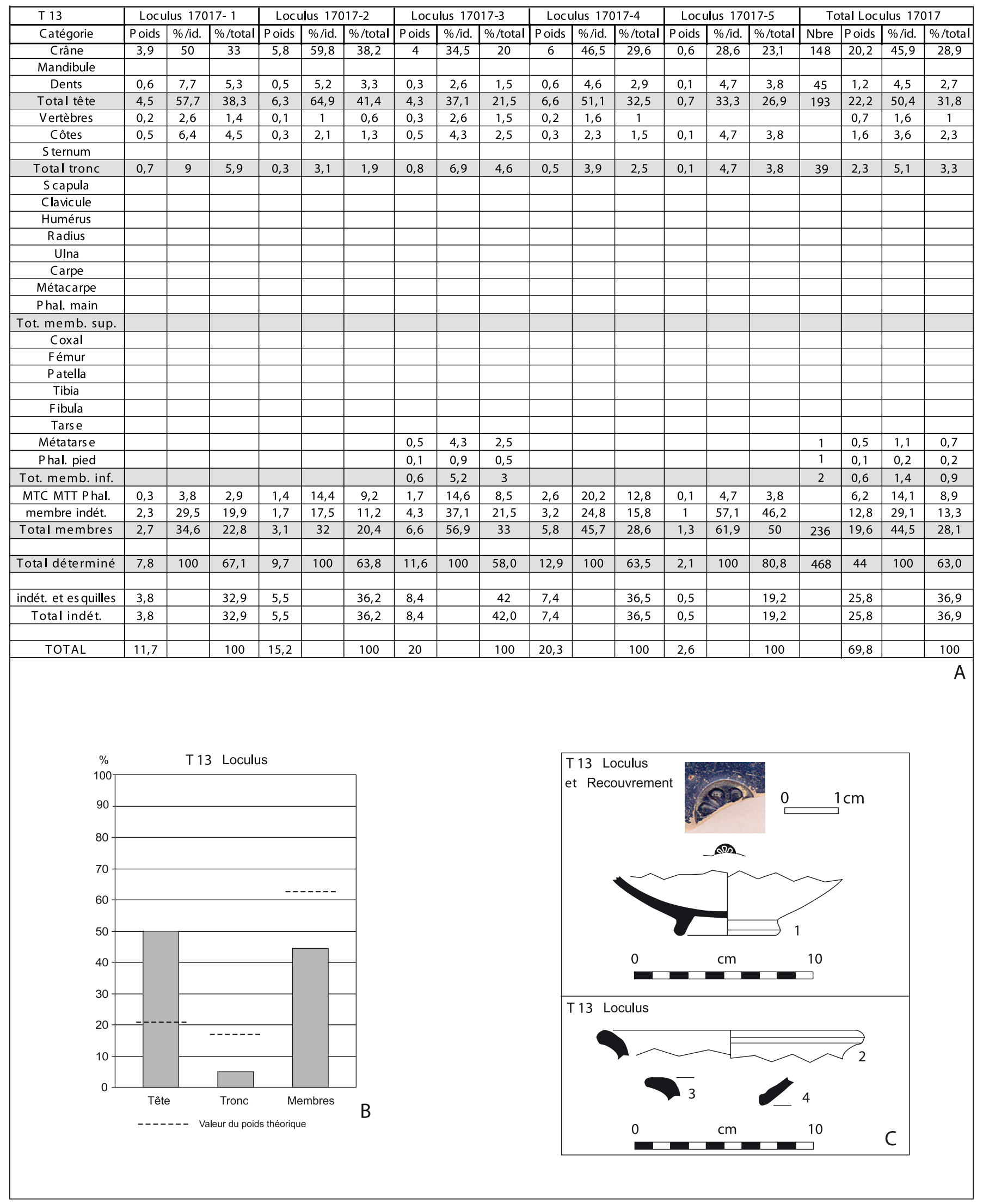

Fig. 20. Tombe T13. A : tableau de détermination des os humains (poids en grammes ; calcul des \% sur la masse). B : répartition du poids des os par grandes régions anatomiques (\% par rapport au total déterminé). C : mobilier (1: céramique à vernis noir ; 2-4 : céramique non tournée). 


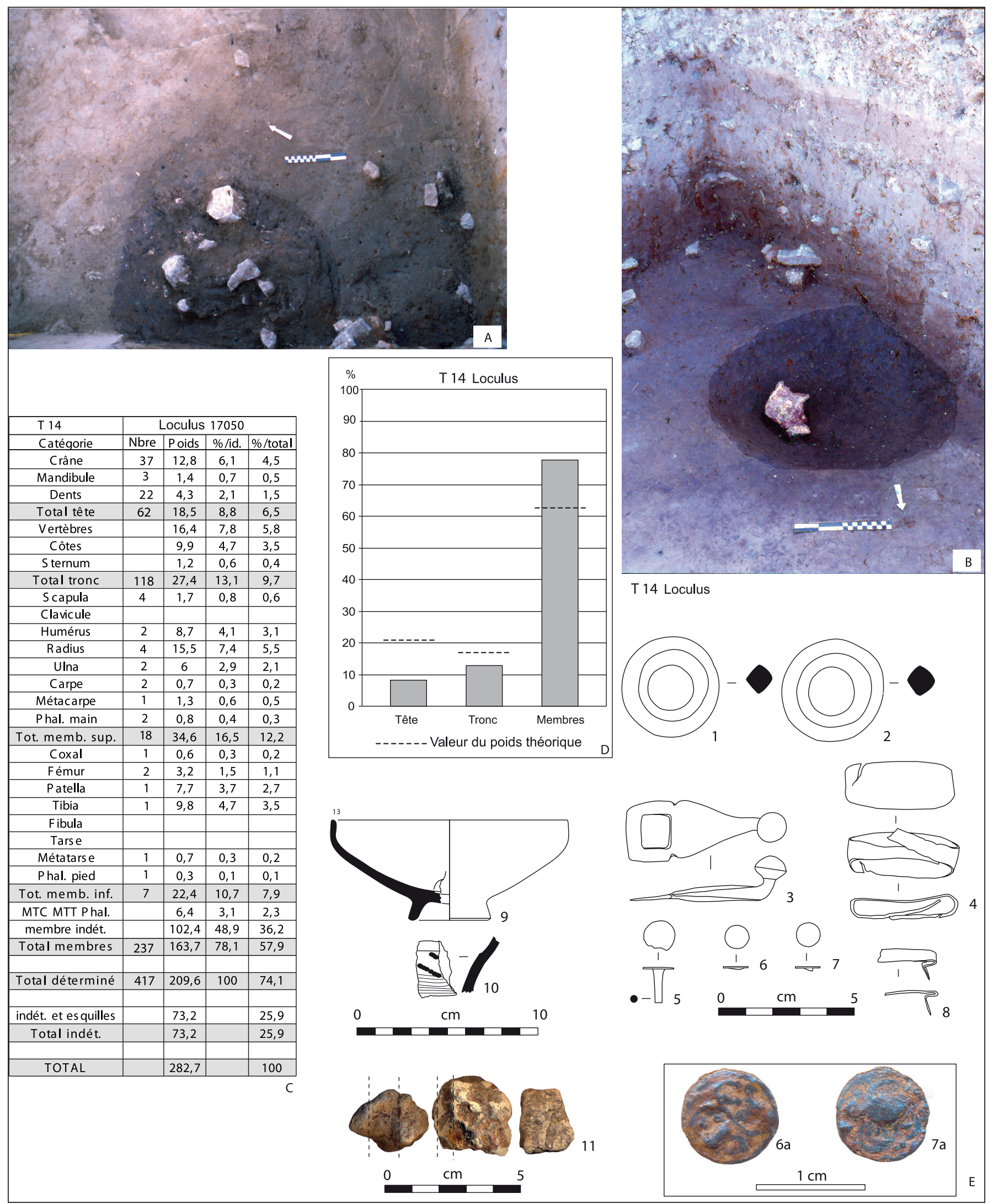

Fig. 21. Tombe T14. A : comblement du loculus vu du sud-ouest. B : loculus en fin de fouille vu du nord. C : tableau de détermination des os humains (poids en grammes ; calcul des \% sur la masse). D : répartition du poids des os par grandes régions anatomiques (\% par rapport au total déterminé). E : mobilier (1-8: fer ; 9 : céramique à pâte claire massaliète ; 10 : céramique non tournée ; 11 : morceaux de torchis). 
Cette sépulture est complète, à l'exception de la portion sud-ouest de la structure de recouvrement surmontée par les murs de l'habitat gallo-romain qui ont limité la fouille dans cette direction (fig. 17, A, B, C et D, et fig. 21, A et B).

\subsection{Le défunt}

Ce sont 282,8 g d'os brûlés qui proviennent du loculus de la tombe T14 (US 17050) (fig. 21, C). D'après la morphologie des os, et en particulier la synostose des extrémités aux corps diaphysaires et celle du listel sur les corps vertébraux, de même que la fermeture des apex des dents, on a affaire à un adulte. Aucun fragment crânien ne montre de suture synostosée ; il pourrait donc s'agir d'un adulte jeune. Par ailleurs, le sexe anthropologique ne peut être précisé.

Le poids d'os contenus dans ce loculus représente $17,4 \%$ du poids moyen d'un squelette d'adulte brûlé (1627,1 g selon McKinley 1993), mais il ne s'agit sans doute pas de la totalité des restes déposés dans cette sépulture, car une partie, au moins, des os livrés par le recouvrement commun aux loculus des tombes T12 et T14 a toutes chances d'appartenir à ce défunt.

C'est $74,1 \%$ du poids total des fragments de ce lot qui a pu être identifié. La sur-représentation des membres est importante, $78,1 \%$ du poids identifié pour un poids théorique de $62,6 \%$; cela se fait surtout au détriment du crâne, très peu représenté ici, $8,8 \%$ du poids identifié au lieu des 20,4\% attendus, tandis que le tronc, $13,1 \%$ du poids identifié, est nettement moins sous-représenté que d'habitude (poids théorique : $17 \%$ ) (fig. 21, D). La couleur blanc crayeux révèle une température de crémation supérieure à $650^{\circ}$ selon le barème de $\mathrm{E}$. Bonnucci et G. Grazziani (1975) et les morceaux de diaphyses sont très souvent fissurés en ondes concentriques, indice d'une incinération d'os frais.

Les os ont été placés dans le loculus hors de tout contenant, irrégulièrement répartis dans l'ensemble du comblement avec des concentrations notables autour des pierres.

Le poids moyen des fragments déterminés, toutes régions anatomiques confondues, est de $0,50 \mathrm{~g}(0,25 \mathrm{~g}$ pour la tête, $0,23 \mathrm{~g}$ pour le tronc et $0,69 \mathrm{~g}$ pour les membres). Pour les 18 fragments identifiés comme provenant des membres supérieurs, le poids moyen est de $1,92 \mathrm{~g}$; pour les 7 fragments identifiés comme provenant des membres inférieurs, il est de $3,20 \mathrm{~g}$.

\subsection{Le loculus}

Le loculus (US 17050) est une cuvette de plan circulaire, de 55 à $60 \mathrm{~cm}$ de diamètre à l'ouverture, aux parois asymétriques, abruptes dans la moitié orientale, en pente douce dans la moitié occidentale et à fond arrondi. Sa profondeur maximale est de $12 \mathrm{~cm}$ (fig. 17, A, B, C et D, et fig. 21, A et B). Le remplissage est composé d'un sédiment limoneux gris très foncé, incluant des charbons de bois épars, en général inférieurs à $0,5 \mathrm{~cm}$ et exceptionnellement plus gros jusqu'à $1 \mathrm{~cm}$, des morceaux de parois en torchis, ayant plus ou moins subi l'action du feu, pouvant atteindre $5 \mathrm{~cm}$ de côté, montrant, pour certains, des empreintes de branches et de brindilles (fig. 21, $\mathrm{C}, \mathrm{n}^{\circ} 11$ ). De nombreuses pierres calcaires non roulées, parfois éclatées au feu, n'excédant pas $10 \mathrm{~cm}$ d'arête mais le plus souvent plus petites, sont présentes de la partie supérieure du comblement jusqu'au fond, surtout au centre de la structure. De même que les restes osseux, le mobilier, plusieurs éléments de la ceinture de suspension et de la poignée d'une épée, sept tessons d'une coupe et quatre tessons de quatre autres vases, ainsi que les restes de torchis se répartissent dans toute l'épaisseur du remplissage du loculus.

\section{Mobilier du loculus}

Objets personnels : armement et fourniment

- Rivet en fer (fig. 21, E, $n^{\circ}$ 5), de garniture de poignée d'une épée ; tête discoïde plate ; tige creuse, dont il manque l'une des extrémités, sans doute pourvue d'une autre tête discoïde ; long. cons. : $14,5 \mathrm{~mm}$; diam. tête : $11,5 \mathrm{~mm}$; diam. tige : $3 \mathrm{~mm}$;

- Deux petits clous en fer (fig. 21, E, $n^{\circ}$ 6,6a, 7 et 7a), probable décoration de fourreau ou de poignée d'épée ; tête discoïde plate et décorée ; sur les deux, arrachement de la tige ; diam. : 8,8 mm ;

- Morceau de ruban en fer replié deux fois ; long. cons. : $21 \mathrm{~mm}$; larg. : $5 \mathrm{~mm}$; ép. :1,7 mm (fig. 21, E, $\mathrm{n}^{\circ} 8$ ) ;

- Quatre éléments de la ceinture de suspension d'un fourreau d'épée :

- deux gros anneaux circulaires en fer (fig. 21, E, $\mathrm{n}^{\circ} 1$ et 2 ), de section losangique ; diam. respectivement : $37 \mathrm{~mm}$ et 37 à $39 \mathrm{~mm}$; haut. respectivement : 10 à $11 \mathrm{~mm}$ et 10 à $12 \mathrm{~mm}$;

- agrafe de ceinture, en fer (fig. 21, E, n 3 et fig. 115); boucle rectangulaire; corps cordiforme ; crochet terminé par un bouton relevé, conique ; long. : $59 \mathrm{~mm}$; larg. : $23 \mathrm{~mm}$;

- passant de courroie en fer (fig. 21, E, $n^{\circ} 4$ ) ; formé d'une fine plaque ovale, prolongée aux deux extrémités par deux pattes très amincies, repliées sur un côté de la plaque; long. : $41 \mathrm{~mm}$; larg. : $17 \mathrm{~mm}$. 
Matériel céramique

Vase réparti dans les tombes $\mathrm{T} 12$ et T14 :

- 7 tessons d'une coupe à pâte claire massaliète de forme CL-MAS 321, presque complète ; pâte grise et couverte gris noir, savonneuse ; le fond porte le cartouche circulaire d'une rosette ; diam. $\max .: 132 \mathrm{~mm}$; haut. : $56 \mathrm{~mm}$; dix autres tessons de ce vase figurent dans la superstructure de recouvrement commune aux loculus des tombes T12 et T14, au nord (us 17027) et au sud-est du loculus (us 17026); l'ensemble de ces fragments représente 80 à $90 \%$ du récipient, et sans doute celui-ci était-il complet lors du bris dans et sur la tombe elle-même (fig. 21, E, n 9).

Tessons de vases isolés :

- 1 tesson d'urne non tournée de forme probable CNT-LOR U5d ; départ du col lissé, épaule décorée d'un rang d'impressions obliques au peigne, panse peignée horizontalement (fig. 21, E, $\mathrm{n}^{\circ} 10$ ) ;

-1 petit tesson de panse peignée de grande urne non tournée (non dessiné) ;

-2 petits tessons de deux grands vases non tournés (non dessinés).

Os d'animaux (détermination A. Gardeisen) :

- 2 fragments de caprinés, non brûlés (talus gauche presque entier (longueur externe : $27,9 \mathrm{~mm}$; épaisseur externe : 15,4 mm); 1 malléole gauche);

-4 fragments carbonisés d'une vertèbre cervicale de capriné ;

- 1 corps d'incisive supérieure de porc, non brûlé ;

- Indéterminés : 3 esquilles brûlées noires et 3 esquilles brûlées blanches.

\section{La structure de recouvrement des tombes T12 et T14}

La structure surmontant les loculus des deux tombes T12 et T14 (US 17016, 17016-1, 17026, 17027 et 17028) est composée du même sédiment d'alluvions limoneuses brunâtres qui recouvre l'ensemble du sol de la nécropole. Elle n'est perceptible que grâce à l'abondant matériel qu'elle contient, dessinant une aire sans doute ovale, orientée NE-SO, mais dont les deux extrémités ne sont pas connues, à cause de la limite de fouille au sud-ouest et par manque ou destruction au nord-est. Cette structure recouvre les deux loculus et déborde largement tout autour, sauf au nord-est. Sa largeur est d'environ $2 \mathrm{~m}$, mais les limites bien évidemment restent floues, tandis que sa longueur excède cette dimension; son épaisseur maximum est de $5 \mathrm{~cm}$ (fig. 17, A et B).

A la base de cette structure, de nombreuses petites pierres calcaires non roulées, provenant de la colline du Dévès, sont éparpillées sur le dessus du comblement des loculus et sur le sol de la nécropole environnant. Aucune ne dépasse $10 \mathrm{~cm}$ d'arête et certaines ont éclaté au feu. Un long amas compact de morceaux d'argile cuite provenant de la paroi d'un four à galette, borde le loculus de la tombe T14 au nord. Plusieurs morceaux, d'une épaisseur de $15 \mathrm{~mm}$, présentent deux surfaces planes ; pour d'autres, d'épaisseur semblable, une surface plane lisse, correspondant à l'extérieur du four, est opposée à une surface gravée de sillons en tous sens (fig. 22, C, $\mathrm{n}^{\circ} 14$ ). Cette portion de four n'a pas été chauffée à cet emplacement car des morceaux rubéfiés jouxtent des secteurs à peine chauffés (fig. 17, A).

Dans toute l'épaisseur du recouvrement prennent place quelques petits charbons de bois mais aussi de très nombreux fragments osseux humains incinérés, épars, pour un poids total de $561,3 \mathrm{~g}$. De nombreux objets mobiliers proviennent des parties surmontant directement les loculus ou leurs abords les plus immédiats, et certains d'entre eux montrent clairement les liens entre le dispositif de recouvrement et les deux loculus :

- au-dessus ou immédiatement au nord-ouest du loculus de la tombe T12 : plusieurs tessons appartenant aux coupes à vernis noir et claire massaliète (fig. 19, $\mathrm{n}^{\circ} 8$ et 13) dont la majeure partie prend place dans le loculus de la tombe T12 (voir supra) ; 44 tessons paraissant appartenir à autant de vases non tournés ;

- immédiatement au nord-ouest du loculus de la tombe T12 : un morceau de bracelet (fig. 22, C, $\mathrm{n}^{\circ} 8$ ), deux clous d'umbo de bouclier (fig. 22, $\mathrm{C}, \mathrm{n}^{\circ} 5$ et 6 ) et un passant de ceinture (fig. 22, $\mathrm{C}, \mathrm{n}^{\circ} 2$ ) ;

- au-dessus du loculus de la tombe T14 : huit tessons appartenant à la coupelle claire massaliète CL-MAS 321 dont des morceaux se trouvent également dans le loculus de la tombe T14 (voir supra) (fig. 21, C, n 9) ; un tesson d'amphore massaliète ;

- au-dessus du loculus de la tombe T14 et immédiatement au nord-ouest, dix-sept tessons de la partie supérieure d'une urne non tournée, soit entre 30 et $40 \%$ du vase (fig. 22, C, $\mathrm{n}^{\circ} 10$ ), répartis en deux amas, un fond appartenant, semble-t-il, à une autre urne non tournée et trois morceaux, soit $80 \%$, d'un couvercle non tourné miniature (fig. 22, C, $\mathrm{n}^{\circ} 12$ ) ;

- au nord-ouest du loculus de la tombe T14, un morceau de fibule en fer (fig. 22, C, $\mathrm{n}^{\circ}$ 9) ;

- au sud-est de ce loculus; un gros anneau en fer de ceinture de suspension d'épée (fig. 22, C, $\mathrm{n}^{\circ} 1$ ), 


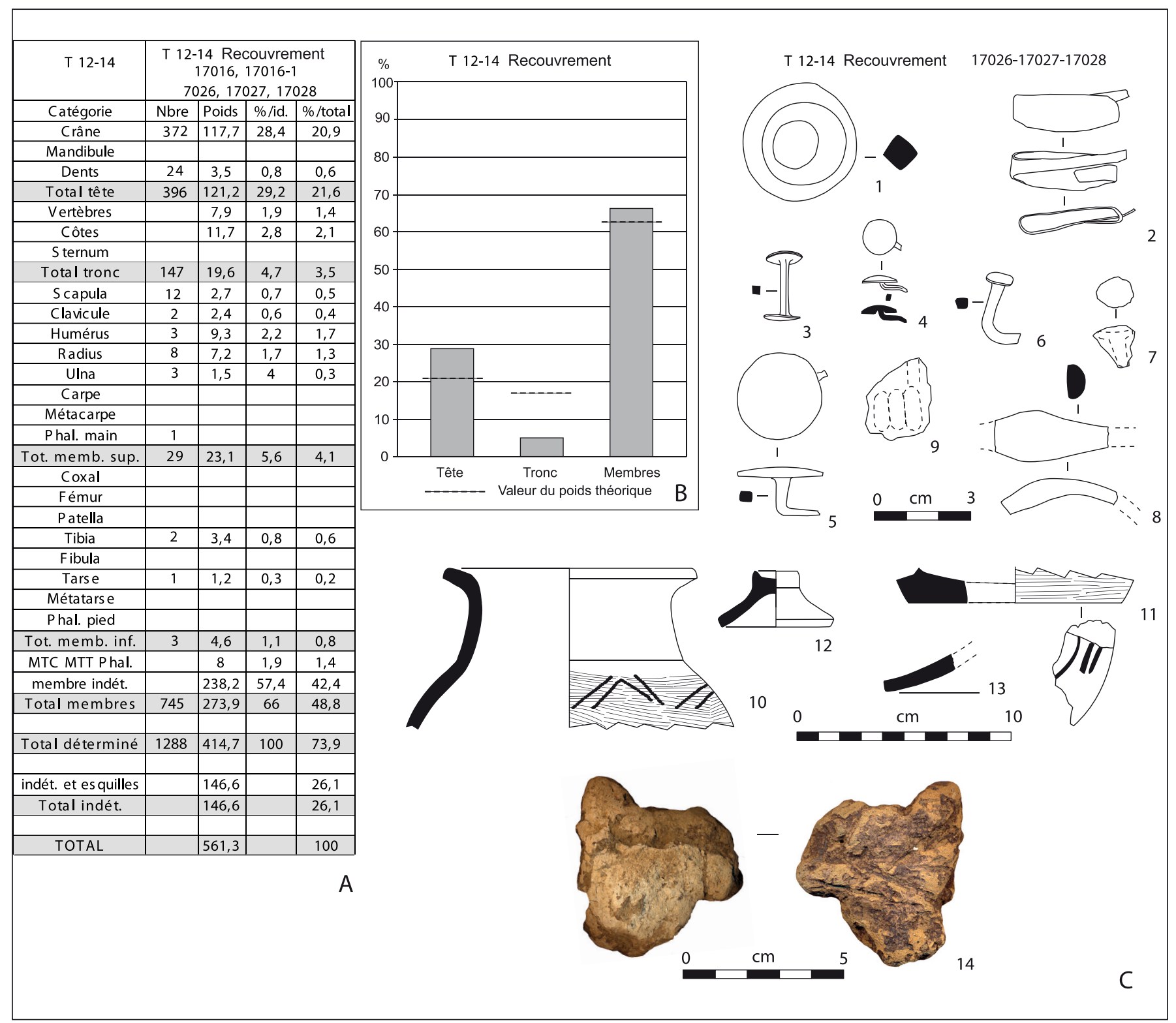

Fig. 22. Recouvrement des tombes 12 et 14. A : tableau de détermination des os humains (poids en grammes; calcul des \% sur la masse). $B$ : répartition du poids des os par grandes régions anatomiques (\% par rapport au total déterminé).

C : mobilier (1-7 et 9 : fer ; 8 : bronze ; 10-13 : céramique non tournée ; 14 : élément de structure en argile cuite).

identique à ceux de ce loculus, deux clous de garniture de poignée d'épée (fig. 22, C, n ${ }^{\circ} 3$ et 4) et un possible rivet d'umbo (fig. 22, $\mathrm{C}, \mathrm{n}^{\circ} 7$ ) ;

- dispersés de part et d'autre du loculus de la tombe T14, à l'ouest et à l'est, vingt-quatre petits tessons non tournés, appartenant vraisemblablement à autant de vases.

\section{Restes osseux humains}

Les restes humains incinérés, au total 561,3 g, se rapportent à de l'adulte, d'après la morphologie des os, les apex des dents fermés, et les sutures synostosées sur la face endocrânienne, non sysnostosées ou en cours de synostose sur la face exocrânienne. Le sexe biologique ne peut être précisé (fig. 22, A).

Les fragments osseux sont plus abondants au-dessus et autour du loculus de la tombe T14 qu'au-dessus et autour de celui de la tombe T12, et un segment de radius situé à proximité du loculus T14 recolle avec un morceau du même os découvert dans ce loculus lui-même. Cependant, il n'existe pas de solution de continuité permettant de séparer deux ensembles pouvant correspondre sûrement à chacun de ces deux sujets. Les dimensions maxima des fragments sont inférieures à 
celles des morceaux déposés dans les loculus, mais cela se note pour toutes les autres tombes de ce secteur de la nécropole. En revanche, l'aspect de ces restes ne diffère pas de ceux des loculus : même couleur et mêmes fissurations en ondes concentriques révélant également une incinération d'os frais à une température semblable. Par ailleurs, le poids moyen des fragments déterminés, toutes régions anatomiques confondues, est de $0,32 \mathrm{~g}$ $(0,31 \mathrm{~g}$ pour la tête, $0,13 \mathrm{~g}$ pour le tronc et $0,37 \mathrm{~g}$ pour les membres). Pour les 29 fragments identifiés comme provenant des membres supérieurs, le poids moyen est de $0,80 \mathrm{~g}$; pour les 3 fragments identifiés comme provenant des membres inférieurs, il est de 1,50 g. Ces valeurs sont tout à fait semblables aux homologues calculées pour les deux loculus. De cette répartition et de cette similitude, on déduira donc que ces os peuvent provenir des deux adultes déposés dans ces deux loculus, sans qu'il soit possible de faire la part de chacun d'eux.

Les taux d'identification sont de 73,9\% du poids total des fragments. Crâne et membres sont sur-représentés : $29,2 \%$ du poids identifié pour le premier (pour un poids théorique de $20,4 \%$ ) et $68,5 \%$ pour les seconds (au lieu des $62,6 \%$ attendus) ; avec $4,7 \%$, le tronc est très nettement sous-représenté (poids théorique : $17 \%$ ) (fig. 22, B).

\section{Mobilier de la structure de recouvrement commune aux tombes T12 et T14}

Objets personnels : armement et fourniment

- Un rivet en fer de garniture de poignée d'épée : deux têtes discoïdes très légèrement bombées, non décorées ; tige de section quadrangulaire ; long. : $21,5 \mathrm{~mm}$; diam. têtes: 11 à $11,5 \mathrm{~mm}$; côté tige : 2,5 à $3 \mathrm{~mm}$ (fig. 22, $\mathrm{C}, \mathrm{n}^{\circ} 3$ ) ;

- Un rivet en fer à tête discoïde légèrement bombée et non décorée ; tige de section quadrangulaire recourbée et cassée ; diam. tête : $11 \mathrm{~mm}$ (fig. 22, C, $\mathrm{n}^{\circ} 4$ ). Il pourrait s'agir d'un rivet de décoration de poignée d'épée ou de fourreau ;

- Un grand rivet de fixation d'ailettes d'umbo de bouclier (fig. 22, C, $\mathrm{n}^{\circ}$ 5), en fer ; tête discoïde légèrement bombée, non décorée (diam. : $27 \mathrm{~mm}$ ) ; tige de section quadrangulaire, repliée à angle droit, déterminant une épaisseur de $10 \mathrm{~mm}$ pour la planche du bouclier ;

- Un petit rivet en fer (fig. 22, C, $\mathrm{n}^{\circ} 6$ ); petite tête discoïde légèrement bombée, non décorée (diam.: $8 \mathrm{~mm}$ ); tige de section quadrangulaire, repliée à angle droit, déterminant une épaisseur de $15 \mathrm{~mm}$ pour l'objet fixé par ce rivet. Il pourrait également s'agir de la fixation d'un umbo de bouclier;

- Un rivet en fer (fig. 22, C, $\mathrm{n}^{\circ} 7$ ), fragmentaire ; cet objet est très corrodé et n'a pas fait l'objet d'une restauration ; il pourrait s'agir d'un élément de fixation d'umbo de bouclier à tête discoïde ;

- Un gros anneau circulaire en fer (fig. 22, C, $\mathrm{n}^{\circ} 1$ ) faisant partie d'une ceinture de suspension d'un fourreau d'épée ; section losangique ; diam. : 35 à $37 \mathrm{~mm}$; haut. : 11 à $14 \mathrm{~mm}$. Cet élément est semblable à ses homologues du loculus 14 ; il s'agit vraisemblablement de la même suspension ;

- Un passant de courroie en fer (fig. 22, C, $n^{\circ} 2$ ) ; formé d'une fine plaque ovale, prolongée aux deux extrémités par deux pattes très amincies, repliées sur un côté de la plaque; long. : 33,5 mm; larg. : $12 \mathrm{~mm}$. Ce passant est de mêmes forme et conception que celui du loculus $14\left(n^{\circ} 4\right)$; quoiqu'un peu plus petit, il doit faire partie de la même ceinture.

Objets personnels : parure et habillement

- Un morceau de grand bracelet en bronze (fig. 22, $\left.\mathrm{C}, \mathrm{n}^{\circ} 8\right)$, portant une nodosité ovale ; section en « $\mathrm{D} »$; haut. : 9 à $16 \mathrm{~mm}$. Cet objet est déformé par l'action d'un feu secondaire; le diamètre semble cependant compris entre 65 et $75 \mathrm{~mm}$;

- Un morceau de ressort de grande fibule en fer (fig. 22, $\mathrm{C}, \mathrm{n}^{\circ}$ 9) (très corrodé et non restauré).

Matériel céramique :

- Des tessons des trois coupes complètes partagées avec les tombes voisines : coupes à vernis-noir et claire massaliète fig. $19, \mathrm{n}^{\circ} 8$ et $\mathrm{n}^{\circ} 13$ du loculus 12 , et coupe claire massaliète fig. $21, \mathrm{C}, \mathrm{n}^{\circ} 9$ du loculus 14 ;

- Un couvercle non tourné miniature, presque complet, de forme CNT-LOR V2a (environ $80 \%$ du vase sont présents) : bord dans la continuité de la paroi rectiligne, lèvre aplatie, petit fond annulaire; surfaces brutes ; diam. $\max .: 55 \mathrm{~mm}$; haut. : $26 \mathrm{~mm}$ (fig. 22, $\left.\mathrm{C}, \mathrm{n}^{\circ} 12\right)$;

- 1 tesson d'urne non tournée du type à panse ovoïde ; base de la panse peignée horizontalement, fond plat avec, à l'extérieur, plusieurs traits incisés larges et peu profonds ; diam. fond: $100 \mathrm{~mm}$ (fig. 22, C, $\mathrm{n}^{\circ} 11$ ) ;

- Portion de la partie supérieure d'une urne non tournée de forme CNT-LOR U5d, variante d, 30 tessons soit environ $40 \%$ du vase : col tronconique rentrant, bord déversé, lèvre aplatie, panse ovoïde à peignage horizontal ; épaule décorée d'impressions au peigne disposées en deux lignes brisées, sur fond de peignage horizontal ; diam. ouv. : $120 \mathrm{~mm}$ (fig. 22, C, $\mathrm{n}^{\circ} 10$ ).

- Tessons de vases isolés :

- 1 tesson de couvercle non tourné de forme CNTLOR V2b : bord dans la continuité de la paroi concave, lèvre aplatie (fig. 22, C, $\mathrm{n}^{\circ} 13$ ) ;

- 4 fragments de bords appartenant à trois urnes non tournées (non dessinés) ; 
- 65 tessons de panses non tournées, correspondant semble-t-il à autant de vases (non dessinés) ;

- 1 fragment d'épaule d'amphore massaliète, avec arrachement d'anse (non dessiné).

Os d'animaux (détermination A. Gardeisen) :

- 3 fragments crâniens de porc juvénile, carbonisés à blanc, se rapportant probablement au crâne du porcelet du loculus de la tombe 12 ;

- 2 fragments de plats de côtes d'un capriné, pouvant également provenir du gril costal identifié dans le loculus de la tombe 12 ;

- 2 fragments d'un équidé adulte mâle ( 1 dent jugale supérieure non brûlée de taille gracile ne présentant pas de pli caballin ; la couronne est cassée mais ce fait peut résulter de l'action du feu (ou de la chaleur), même si l'émail n'en porte pas de trace ; 1 racine de canine en partie chauffée, ayant pu se casser sous l'effet de la chaleur.) ;

- 2 fragments de porcs (fragment d'émail dentaire, cf. molaire ; fragment de coxal gauche (acétabulum), brûlé, blanc sur le bord ventral et noir sur le bord dorsal);

-2 esquilles crâniennes brûlées d'un animal de petite taille ;

- 3 fragments de diaphyses d'os longs d'un animal de petite taille ;

- fragment de diaphyse d'os long d'un animal de grande taille, brûlée, noire ;

- Indéterminés : 33 esquilles brûlées ; 6 esquilles non brûlées ;

- Malacofaune : fragment de bord de coquillage.

\section{La tombe T15}

\section{1. État et composantes de la tombe}

La tombe T15 a accueilli un adulte de sexe non déterminé. Elle comprend un loculus, qui a pu être entièrement fouillé, mais aucune trace d'un éventuel recouvrement n'a été décelée. Ce loculus n'a cependant pas été arasé car le sol de la nécropole est bien marqué à ses environs immédiats (fig. 23, A et B).

\subsection{Le défunt}

Au défunt se rapportent 101,8 g d'os brûlés, provenant tous du loculus (US 17033). La détermination de l'âge adulte repose sur la morphologie des os et la fermeture des apex des dents. Les sutures sont libres sur la face exocrânienne et en cours de synostose sur l'endocrâne. Il s'agit donc d'un adulte peut-être âgé de plus de trente ans, mais pas un vieillard. Le sexe anthropologique ne peut pas être précisé (fig. 23, C).

Le poids d'os représente $16 \%$ du poids moyen d'un squelette d'adulte brûlé (1627,1 g selon McKinley 1993). Les taux d'identification sont de $58 \%$ du poids total des fragments. La sur-représentation des membres est importante, $83,7 \%$ du poids identifié pour un poids théorique de $62,6 \%$; cela se fait au détriment du crâne, $14,6 \%$ du poids identifié au lieu des $20,4 \%$ attendus, et surtout du tronc, très nettement sous-représenté, $1,7 \%$ (poids théorique : $17 \%$ ) (fig. 23, D). La couleur blanc crayeux est l'indice d'une température de crémation supérieure à $650^{\circ}$ selon le barème de $\mathrm{E}$. Bonnucci et $\mathrm{G}$. Grazziani (1975) et la fissuration en ondes concentriques des morceaux de diaphyses marque une incinération d'os frais. Les restes osseux ont été déposés dispersés dans le loculus, hors de tout contenant.

Le poids moyen des fragments déterminés, toutes régions anatomiques confondues, est de $0,33 \mathrm{~g}(0,30 \mathrm{~g}$ pour la tête, $0,10 \mathrm{~g}$ pour le tronc et $0,36 \mathrm{~g}$ pour les membres). Pour les 3 fragments identifiés comme provenant des membres inférieurs, il est de $1,54 \mathrm{~g}$.

\subsection{Le loculus}

Le loculus est une grande cuvette de plan ovalaire, de $1,5 \mathrm{~m}$ de long sur $0,5 \mathrm{~m}$ de large, mais très peu profonde, 2 à $3 \mathrm{~cm}$ seulement (US 17033). Elle est remplie d'un sédiment limoneux gris jaunâtre contenant, éparpillés sur toute sa surface, des nodules d'argile chauffée, quelques rares petits morceaux de charbons de bois, des os humains incinérés très fragmentés et un mobilier peu abondant, avec notamment au sud, plusieurs éléments d'objets en bronze et en fer (fig. 23, A et B).

\section{Mobilier du loculus}

Objets personnels: parure et habillement :

- Plusieurs fragments d'une grande fibule en bronze et corail (fig. 23, E, n ${ }^{\circ} 1$ ) : moitié de ressort bilatéral à six ou huit spires et corde externe ; morceau d'arc filiforme ; morceaux du porte-ardillon ; cabochon en corail appartenant à la décoration du pied, de forme ovale, percé au centre d'un trou de rivet, orné de trois demicercles concentriques en creux opposés ; larg. rest. au ressort: env. $35 \mathrm{~mm}$; axes du cabochon : 8 à $10 \mathrm{~mm}$. La couleur blanchâtre du corail montre une exposition 
au feu. Il s'agit d'une fibule du type à pied replié vers l'arc et terminé par un petit disque sur lequel devait être riveté le cabochon (type $11 \mathrm{de} \mathrm{C}$. Tendille).

- Quatre segments d'un ruban en bronze, de section lenticulaire, déformé par le feu ; larg. : 9 à $12 \mathrm{~mm}$; ép. : $4 \mathrm{~mm}$; il s'agit peut-être d'un bracelet (fig. 23, E, $\left.\mathrm{n}^{\circ} 2\right)$.

Tessons de vases isolés :

- 1 petit tesson de vase à vernis noir de l'atelier de Rosas, de forme non déterminable ;

- 3 tessons de grande urne non tournée de forme probable CNT-LOR U5d, ne représentant qu'une très petite partie du récipient : col tronconique rentrant à surface lissée, panse ovoïde peignée horizontalement ; épaule décorée d'impressions de coups de peigne obliques; diam. max. estimé : env. 220 mm (fig. 23, E, n ${ }^{\circ}$ ) ;

-1 tesson de fond plat de grande urne non tournée (fig. 23, E, $\mathrm{n}^{\circ} 4$ ), peut-être même exemplaire que le précédent ;

- 1 bord déversé d'urne non tournée (fig. $23, \mathrm{E}, \mathrm{n}^{\circ}$ 5) ;

- 1 bord déversé à lèvre aplatie d'urne non tournée de forme probable CNT-LOR U5d (fig. 23, E, n ${ }^{\circ} 6$ ) ;

- 22 tessons informes non tournés, correspondant vraisemblablement à autant de vases (non dessinés).

Os d'animaux (détermination A. Gardeisen) :

- Caprinés : 3 fragments de vertèbre thoracique et 1 fragment d'émail dentaire ;

- Indéterminés : 1 esquille brûlée, noire, et 5 esquilles non brûlées ;

- Microfaune : 1 vertèbre (cf. reptile) ; 1 fragment de phalange ou de vertèbre caudale brûlée.

\section{La tombe T16}

\section{1. État et composantes de la tombe}

C'est la tombe d'un adulte de sexe non déterminé. Elle se compose d'un loculus, entièrement fouillé, et de quelques restes, très localisés, du dispositif de recouvrement (fig. 24, A, B et C).

\subsection{Le défunt}

Ce défunt est attesté par 155,6 g d'os brûlés, éparpillés dans le loculus (US 17030-2), hors de tout contenant.
La détermination de l'âge adulte repose sur la morphologie des os et la fermeture des apex des dents. Le sexe anthropologique ne peut pas être précisé (fig. 24, D).

Les os sont très fragmentés, et peu d'éléments sont identifiables, 48,8 \% seulement du poids total. Le crâne et plus encore le tronc sont sous-représentés : respectivement $13,6 \%$ et $3 \%$ du poids total déterminé contre $20,4 \%$ et $17 \%$ en théorie. Avec $83,4 \%$ (pour $62,6 \%$ attendus) les membres sont largement prédominants (fig. 24, E).

L'incinération a affecté des os frais, comme le montrent les fissurations en ondes concentriques des diaphyses. Presque tous les restes ont une couleur blanc crayeux, traduisant une température de crémation de l'ordre de $650^{\circ}$ selon le barème de $\mathrm{E}$. Bonnucci et G. Grazziani (Bonucci, Grazziani 1975).

Le poids moyen des fragments déterminés, toutes régions anatomiques confondues, est de $0,36 \mathrm{~g}(0,30 \mathrm{~g}$ pour la tête, $0,22 \mathrm{~g}$ pour le tronc et $0,40 \mathrm{~g}$ pour les membres). Pour le seul fragment identifié comme provenant d'un membre supérieur, le poids est de 1,61 g et pour le seul fragment identifié comme provenant d'un membre inférieur, il est de $2,07 \mathrm{~g}$.

\subsection{Le loculus}

Le loculus (US 17030-2) est une cuvette de plan ovalaire, large mais peu profonde, mesurant $1,7 \mathrm{~m}$ de long sur $1 \mathrm{~m}$ de large et $4 \mathrm{~cm}$ de profondeur maximum. Son fond est irrégulier. Le comblement est constitué par un sédiment alluvial de couleur gris jaunâtre. Il inclut quelques pierres calcaires non roulées, n'excédant pas $10 \mathrm{~cm}$ d'arête et montrant parfois des traces de feu. En revanche, il est dépourvu de nodules d'argile cuite ou chauffée, ce qui le distingue nettement du remplissage de la tombe précédente. La densité des os humains incinérés diminue du centre vers la périphérie, tandis que, dans la même direction, augmente la fragmentation des restes. Les rares charbons de bois, toujours de petites dimensions, inférieurs à $5 \mathrm{~mm}$, montrent une répartition semblable. Le mobilier se compose de 43 tessons appartenant à 39 récipients différents, non tournés à une exception près. Aucun objet métallique ne figure dans le dépôt (fig. 24, A, B et C).

\section{Mobilier du loculus}

Tessons de vases isolés :

- 1 tesson de panse de coupe claire massaliète, à pâte de couleur grise, de forme non déterminable (non dessiné) ; 


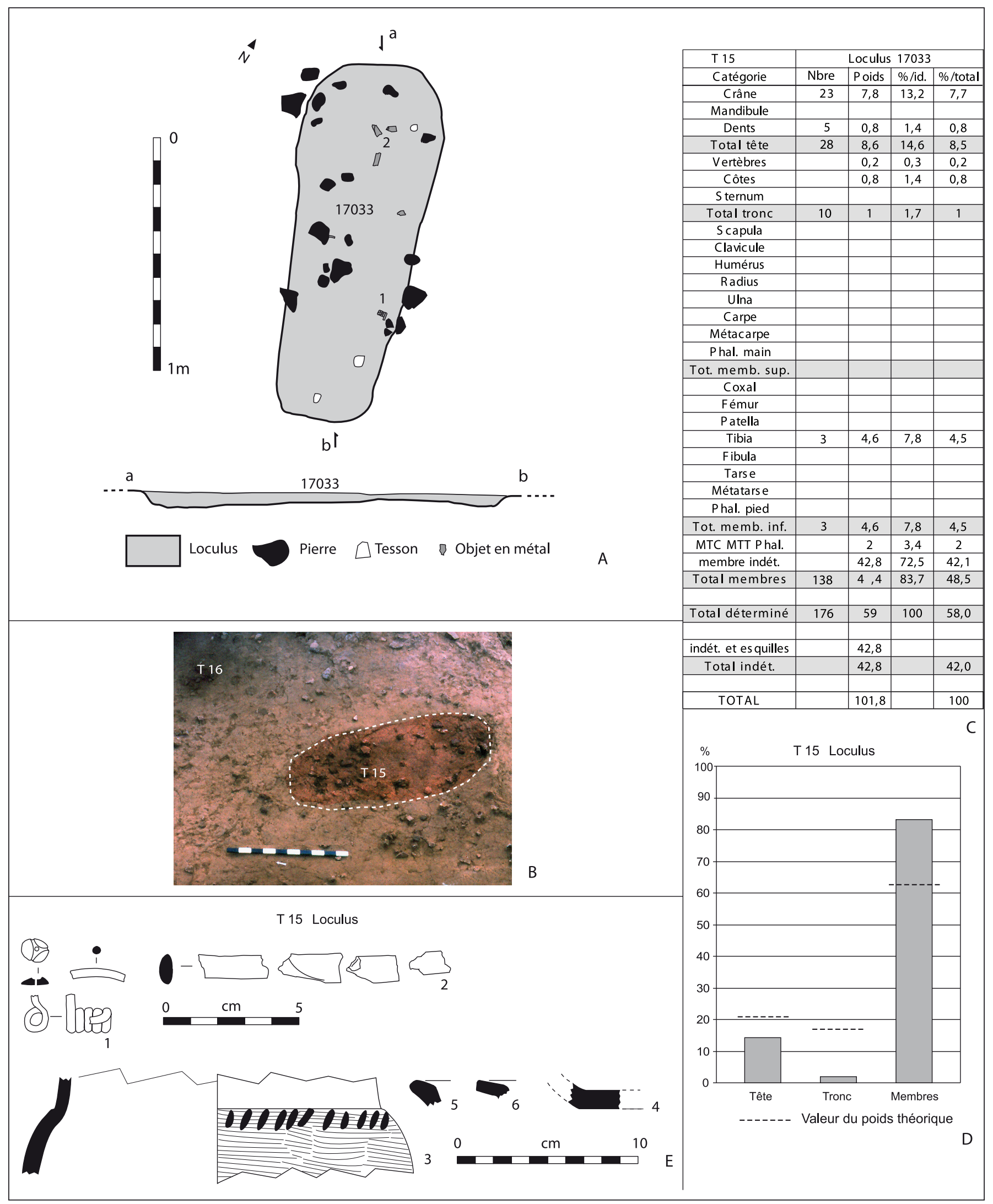

Fig. 23. Tombe T15. A : plan et coupe. B : loculus vu du sud-ouest. C : tableau de détermination des os humains (poids en grammes ; calcul des $\%$ sur la masse). $\mathrm{D}$ : répartition du poids des os par grandes régions anatomiques (\% par rapport au total déterminé).

$\mathrm{E}$ : mobilier (1 : fibule en bronze et corail ; 2 : bronze ; 3-6 : céramique non tournée). 


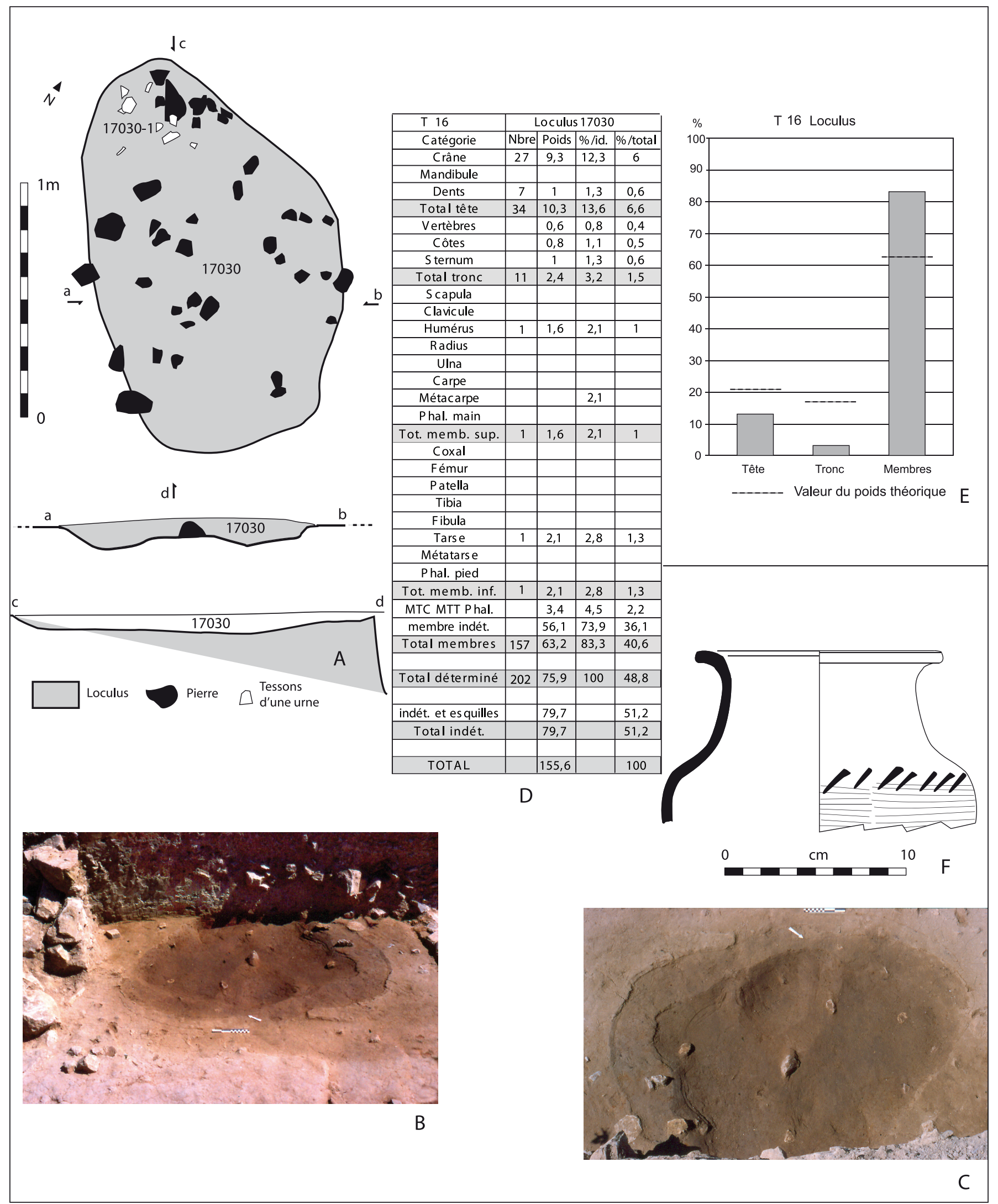

Fig. 24. Tombe T16. A : plan et coupes. B : remplissage du loculus, vu du sud-ouest. C : loculus en fin de fouille, vu du sud-ouest. D : tableau de détermination des os humains (poids en grammes ; calcul des \% sur la masse). E : répartition du poids des os par grandes régions anatomiques (\% par rapport au total déterminé). F : mobilier (céramique non tournée). 
- 5 tessons de la panse d'une urne non tournée (non dessinés) ;

- 1 tesson de l'épaule d'une urne non tournée (non dessiné) ;

- 36 tessons informes de céramique non tournée provenant, semble-t-il, d'autant de vases (non dessinés).

Os d'animaux (détermination A. Gardeisen) :

- 1 fragment dorsal de diaphyse de métatarse de capriné (cf. mouton) ;

-1 fragment de plat de côte d'un animal de petite taille ;

- Indéterminés : 11 esquilles brûlées, noires, et 12 esquilles non brûlées ;

- Malacofaune : 1 fragment de coquille de moule.

\subsection{Le dispositif de recouvrement}

La seule trace conservée de la structure de recouvrement de la tombe consiste dans la présence d'une quinzaine de tessons d'une urne non tournée gisant à plat sur la surface supérieure du comblement du loculus, près de son extrémité ouest (fig. 24, A, 17030-1).

\section{Mobilier du dispositif de recouvrement}

Matériel céramique :

- Portion de la partie supérieure d'une urne non tournée de forme CNT-LOR U5d, variante $\mathrm{d}, 15$ tessons représentant environ $10 \%$ du récipient: col tronconique rentrant à surface lissée, bord déversé avec méplat vers l'intérieur, panse ovoïde peignée horizontalement, épaule décorée d'une rangée de coups incisés obliques ; diam. max. : $170 \mathrm{~mm}$ (fig. 24, F).

\section{La tombe T17}

\section{1. État et composantes de la tombe}

La tombe T17 abrite un seul défunt incinéré, un adulte de sexe non déterminable. Entièrement fouillée, elle se compose d'un loculus (US 17053), d'un dispositif enterré annexe (US 17115), et d'une structure de recouvrement s'étendant largement tout autour (US 17029) (fig. 25, A, B et C).

\subsection{Le défunt}

Le défunt, un adulte, est représenté par 413,4 g d'os brûlés. L'estimation de l'âge repose sur divers indices, la morphologie des os, le listel soudé sur les corps vertébraux, la fermeture de l'apex des racines de dents conservées et notamment d'une troisième molaire inférieure. Le sexe anthropologique ne peut pas être précisé (fig. 26, A).

Le loculus a livré $153.4 \mathrm{~g}$ d'os et la structure de recouvrement, $260 \mathrm{~g}$, et les deux lots sont tout à fait compatibles. Dans les deux cas, les restes osseux ont une couleur blanc crayeux qui révèle une température de crémation supérieure à $650^{\circ}$ selon le barème de E. Bonnucci et G. Grazziani (1975) et les morceaux de diaphyses sont très souvent fissurés en ondes concentriques, indice d'une incinération d'os frais. Qu'il s'agisse du loculus ou de la superstructure, les restes osseux sont dispersés, hors de tout contenant, et aucune disposition particulière des fragments n'a été perçue. Toutefois, ceux-ci sont très rares dans la partie inférieure du remplissage du loculus $(4,2 \mathrm{~g}$ dans les trois décapages du fond, décapages 3 à 5) et deviennent de plus en plus abondants vers le haut $(20,1 \mathrm{~g}$ à mi-hauteur, dans le décapage 2 , et $128,8 \mathrm{~g}$ en haut, dans le décapage 1).

Le poids d'os déposés dans le loculus représente 9,4\% du poids moyen d'un squelette d'adulte brûlé, $1627,1 \mathrm{~g}$ selon McKinley (1993) et celui des os contenus dans le recouvrement, $16 \%$, soit au total, $25,4 \%$. Les taux d'identification des pièces sont comparables pour le loculus et la superstructure, respectivement 73,3 et $75,7 \%$ du poids total des fragments. Dans le loculus, le tronc est sous-représenté : 10,8\% du poids identifié (poids théorique : $17 \%$ ) ; il en va de même, ce qui est plus rare, et avec un déficit plus important, du crâne : $8,7 \%$, au lieu des 20,4\% attendus ; avec 80,5\%, les membres, évidemment, dépassent la valeur théorique $(62,6 \%)$. A l'intérieur du loculus, la ventilation de ces proportions par décapage n'est pas significative car les effectifs sont trop inégaux. Le lot du recouvrement montre, pour sa part, une répartition quelque peu différente : avec $21 \%$ du poids d'os identifiés, le crâne est normalement représenté, avec 3,2 \%, le tronc est fortement sous-représenté, et ce déficit est compensé par les membres $(75,8 \%)$ (fig. 26, B).

Le poids moyen des fragments déterminés, toutes régions anatomiques confondues, est semblable dans le loculus et dans le dispositif de recouvrement et s'élève à $0,31 \mathrm{~g}$ pour le premier et $0,33 \mathrm{~g}$ pour le second (provenant du loculus : $0,24 \mathrm{~g}$ pour la tête, $0,17 \mathrm{~g}$ pour le tronc et $0,36 \mathrm{~g}$ pour les membres; provenant du recouvrement : $0,26 \mathrm{~g}$ pour la tete, $0,27 \mathrm{~g}$ pour le tronc et $0,37 \mathrm{~g}$ pour les membres). Pour les 6 fragments identifiés comme provenant des membres supérieurs, le poids moyen est de 
$0,11 \mathrm{~g}$; pour le seul fragment identifié comme provenant d'un membre inférieur, il est de 2,80 g.

\subsection{Le loculus}

Le loculus (US 17053) présente, à l'ouverture, un plan circulaire de $50 \mathrm{~cm}$ de diamètre. C'est une cuvette profonde de $12 \mathrm{~cm}$ au maximum au centre, à fond arrondi et à la paroi abrupte marquée par un net ressaut à mi-hauteur au nord (fig. 25, A et B).

Le remplissage comprend deux strates. Au fond prend place une couche de sédiment gris sombre dont le sommet se trouve au niveau du ressaut de la paroi nord du loculus (décapages 17053-3 à 5). Ce sédiment enrobe trois pierres verticales qui affleurent le sommet de ce niveau. Il inclut de rares petits morceaux de charbons de bois et de très rares fragments osseux humains incinérés.

La strate supérieure est formée d'un sédiment noir incluant de petits morceaux de charbons de bois (décapages 17053-1 et 2). Plusieurs pierres calcaires centimétriques, éclatées au feu, reposent à sa base, sur le sommet du niveau précédent (décapages 17053-2). Trois pierres calcaires de 3 à $5 \mathrm{~cm}$ d'arête, montrant des traces de passage au feu, et plusieurs éclats prennent place dans la partie supérieure de cette couche (décapage 17053-1).

Les morceaux d'os humains brûlés, souvent de grandes dimensions, éparpillés, abondent dans ce niveau. Ils sont toutefois beaucoup moins nombreux dans le décapage inférieur 17053-2 (20,1 g) que dans le décapage supérieur 17053-1 (128,8 g). C'est dans ce dernier que figurent les seuls objets mobiliers retrouvés dans le loculus et qui portent des traces de passage sur le bûcher, morceaux d'un anneau en bronze (fig. 25, D, $n^{\circ} 1$ ) et d'une lamelle en fer (fig. 25, D, $n^{\circ} 2$ ).

\section{Mobilier du loculus}

Objets métalliques indéterminés :

- Deux morceaux d'un anneau en bronze; section en D ; diam. ext. : $22 \mathrm{~mm}$; haut. : $3 \mathrm{~mm}$ (fig. 25, D, $\left.\mathrm{n}^{\circ} 1\right)$;

- Quatre morceaux de lamelle de fer recourbée ; ép. : $1 \mathrm{~mm}$; il s'agit peut-être d'une bague de serrage d'emmanchement (fig. 25, D, $\mathrm{n}^{\circ} 2$ ).

Os d'animaux (détermination A. Gardeisen) :

- Indéterminés : 5 esquilles carbonisées, de couleur blanc-bleu ;

- Malacofaune : petit lot d'escargots terrestres.

\subsection{Le dispositif enterré annexe (US 17115 et 17116)}

Le dispositif annexe US 17115 et 17116 se trouve à $20 \mathrm{~cm}$ du bord nord du loculus US 17053. Il se compose d'une fosse profonde (US 17115) dont les limites ne peuvent être appréciées, approximativement, que par les grosses pierres de calcaire local (US 17116) qu'elle renferme, car le sédiment de son comblement est exactement le même que celui des environs, des alluvions limoneuses gris jaunâtre (fig. 25, A et C).

Ces pierres présentent une disposition particulière. L'élément principal est constitué par deux blocs parallélépipédiques allongés, superposés verticalement dans la moitié orientale du dispositif. La pierre du fond (US 17116-2) mesure $24 \mathrm{~cm}$ de long sur $12 \mathrm{~cm}$ de large. Elle est surmontée par un bloc plus important (US 17116-1), de $48 \mathrm{~cm}$ de long, $25 \mathrm{~cm}$ de large et $16 \mathrm{~cm}$ d'épaisseur. Ce dernier élément a une forme régulière mais seule la surface plane de son sommet semble avoir été aménagée. Il émerge de $8 \mathrm{~cm}$ au-dessus du sol de la nécropole. Ces deux blocs forment une sorte de pilier vertical de $73 \mathrm{~cm}$ de hauteur, presque entièrement enterré. Ce dispositif est calé, à l'ouest, par six blocs décimétriques, irréguliers et bruts, en position verticale, à l'exception de ceux qui sont placés en haut du comblement, en position horizontale. L'ensemble de ces pierres implique le creusement d'une fosse de plan ovalaire, d'au moins 60 et $40 \mathrm{~cm}$ d'axes à l'ouverture et d'une profondeur minimum de $65 \mathrm{~cm}$, avec paroi verticale dans sa moitié est et oblique très prononcée dans sa moitié ouest. A part quelques très rares menus charbons de bois dans la partie supérieure du comblement, le sédiment enrobant ces pierres ne renferme aucun document, os ou objet. Il en va de même dans le niveau situé audessous de cet amas de blocs.

\subsection{La structure de recouvrement (US 17029)}

La structure de recouvrement (US 17029) est constituée par une nappe d'alluvions limoneuses de couleur gris clair jaunâtre, de plan ovale, de $2 \mathrm{~m}$ sur l'axe SO-NE et 1,5 $\mathrm{m}$ sur l'axe SE-NO, d'une épaisseur de $6 \mathrm{~cm}$ maximum, incluant de très nombreux os humains incinérés (fig. 25, A). Ce sédiment ne se différencie de celui qui surmonte l'ensemble du sol de la nécropole que par la présence de ces morceaux d'os, éparpillés dans toute son épaisseur. Localement ceux-ci sont plus abondants, notamment dans deux secteurs au nord et à l'ouest du loculus. Cet épandage contient également 29 tessons épars, appartenant à autant de vases différents. 


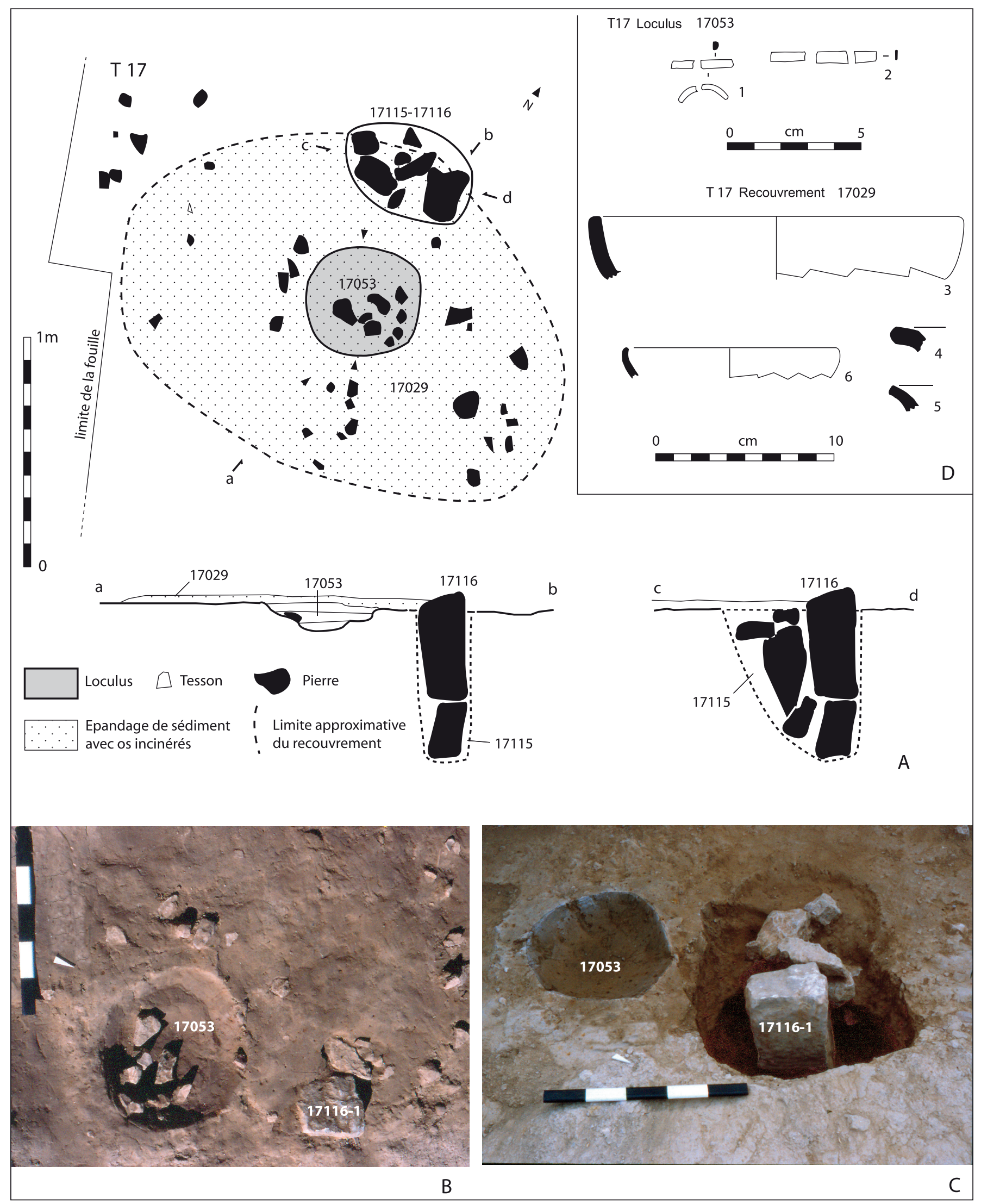

Fig. 25. Tombe T17. A : plan et coupes. B : loculus fouillé et structure annexe, vus du sud-ouest. C : loculus et structure annexe en fin de fouille, vus du sud-ouest. $\mathrm{D}$ : mobilier ( 1 : bronze ; 2 : fer ; $3-5$ : céramique non tournée ; 6 : céramique à vernis noir). 


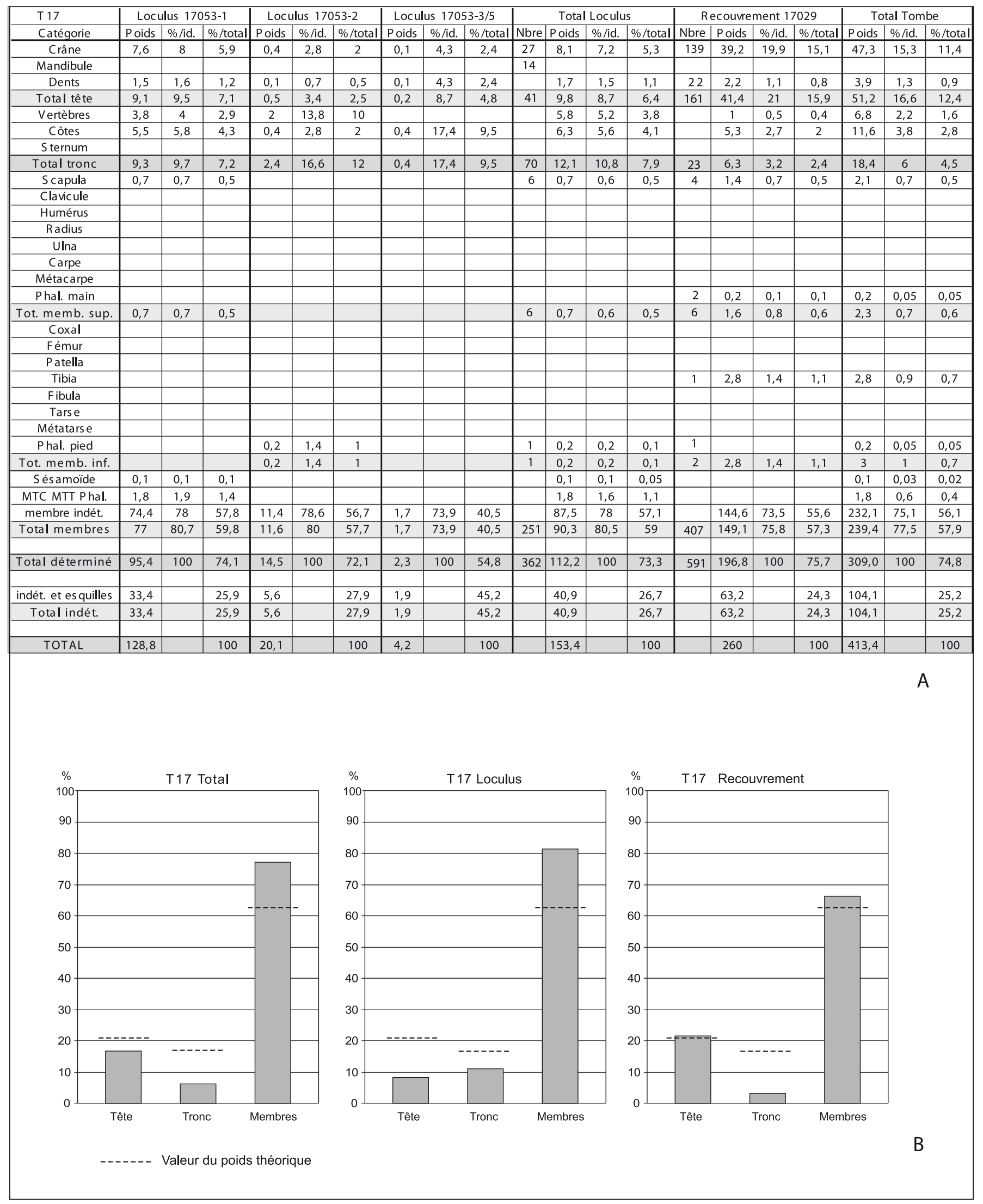

Fig. 26. Tombe T17. A : tableau de détermination des os humains (poids en grammes; calcul des $\%$ sur la masse). $\mathrm{B}$ : répartition du poids des os par grandes régions anatomiques (\% par rapport au total déterminé). 
Il recouvre le comblement du loculus US 17053 et déborde largement tout autour; il s'étend aussi sur le dessus des pierres de la fosse annexe US 17115, mais laisse apparaître, du moins lors de la fouille, le sommet de la grande pierre verticale de cette fosse (US 17116-1).

\section{Mobilier de la structure de recouvrement}

Tessons de vases isolés :

- 1 tesson de bord de bol à vernis noir de l'atelier de Rosas, de forme Lamb. 27 (= forme Puig 11.a); diam. ouv. : $120 \mathrm{~mm}$ (fig. 25, $\mathrm{D}, \mathrm{n}^{\circ}$ 6) ;

-3 petits tessons de panse d'un autre récipient à vernis noir de l'atelier de Rosas, de forme non déterminable (non dessiné) ;

- 1 tesson de panse de vase fermé, urne ou œnochoé, à pâte claire massaliète (non dessiné) ;

- 1 tesson d'urne non tournée : bord déversé à lèvre arrondie (fig. 25, $\mathrm{D}, \mathrm{n}^{\circ} 4$ ) ;

- 1 tesson d'urne non tournée : bord déversé à lèvre aplatie (fig. 25, D, $\mathrm{n}^{\circ} 5$ ) ;

- 1 tesson de coupe non tournée CNT-LOR C1: panse arrondie-convexe, bord divergent à lèvre arrondie ; diam. max. : env. $210 \mathrm{~mm}$ (fig. 25, $\mathrm{D}, \mathrm{n}^{\circ} 3$ ) ;

-23 tessons de vases non tournés, urnes à panse peignée et coupes, appartenant probablement à autant de vases.

Os d'animaux (détermination A. Gardeisen) :

- caprinés : 1 molaire; 1 fragment de diaphyse de métapode carbonisé, noir ;

- 1 fragment indéterminé d'épiphyse d'os long d'animal de petit taille ;

- Indéterminés : 5 esquilles brûlées, noires, 7 esquilles carbonisées de couleur blanc-bleu et 14 esquilles non brûlées ;

- Malacofaune :1 fragment de coquillage marin.

\section{La tombe T18}

\section{1. État et composantes de la tombe}

La tombe T18 abrite les restes d'un grand enfant, adolescent ou adulte, de sexe indéterminé. Elle se compose d'un loculus (US 17035), entièrement fouillé, mais aucune trace d'une éventuelle structure de recouvrement n'a été observée; cependant, la partie supérieure du loculus n'a pas été tronquée car le paléosol est conservé aux abords immédiats (fig. 27, A).

\subsection{Le défunt}

Du défunt, proviennent $121 \mathrm{~g}$ d'os brûlés, éparpillés dans le loculus, hors de tout contenant (fig. 27, B). Son âge ne peut être précisé ; il s'agit d'un grand enfant ou d'un individu de taille adulte, mais pas un jeune enfant ; le sexe anthropologique n'est pas non plus appréciable ; toutefois, parmi les rares pièces l'accompagnant, la présence d'éléments d'armes et de fourniment plaide en faveur d'un individu masculin.

Ces restes sont très fragmentés, et peu d'éléments sont identifiables (56,5\% seulement du poids total d'os). Le crâne est absent, le tronc représente $1,8 \%$ du poids total d'os déterminés et les membres 98,2\% (fig. 27, C).

L'incinération a affecté des os frais comme l'indiquent les fissurations en ondes concentriques des diaphyses. Presque tous les fragments osseux sont de couleur blanc crayeux, traduisant une crémation vers $650^{\circ}$ selon le barème de E. Bonnucci et G. Grazziani, mais quelques-uns néanmoins montrent une couleur noire attestant une température plus basse par endroits (Bonucci, Grazziani 1975).

Le poids moyen des fragments déterminés, toutes régions anatomiques confondues, est de $0,39 \mathrm{~g}(0,17 \mathrm{~g}$ pour le tronc et $0,40 \mathrm{~g}$ pour les membres). Pour les 5 fragments identifiés comme provenant des membres supérieurs, le poids moyen est de 1,26 g; pour les 3 fragments identifiés comme provenant des membres inférieurs, il est de $2,53 \mathrm{~g}$.

\subsection{Le loculus}

Une cuvette de plan ovalaire, large et peu profonde, constitue le loculus (US 17035). Elle mesure 1,4 m de long sur $0,9 \mathrm{~m}$ de large et 2 à $3 \mathrm{~cm}$ de profondeur (fig. 27, A). Elle est remplie d'un sédiment limoneux gris foncé à noirâtre. Celui-ci contient, dispersées, quelques pierres calcaires non roulées, ne dépassant pas $7 \mathrm{~cm}$ de côté, certaines présentant des éclatements thermiques. Les os humains incinérés, quelques rares petits morceaux de charbons de bois et un mobilier peu abondant sont dispersés dans l'ensemble de ce remplissage.

\section{Mobilier du loculus}

Objets personnels : armement et fourniment

- Un rivet de fixation d'ailette d'umbo de bouclier, en fer ; tête discoïde, légèrement creusée dans sa partie 


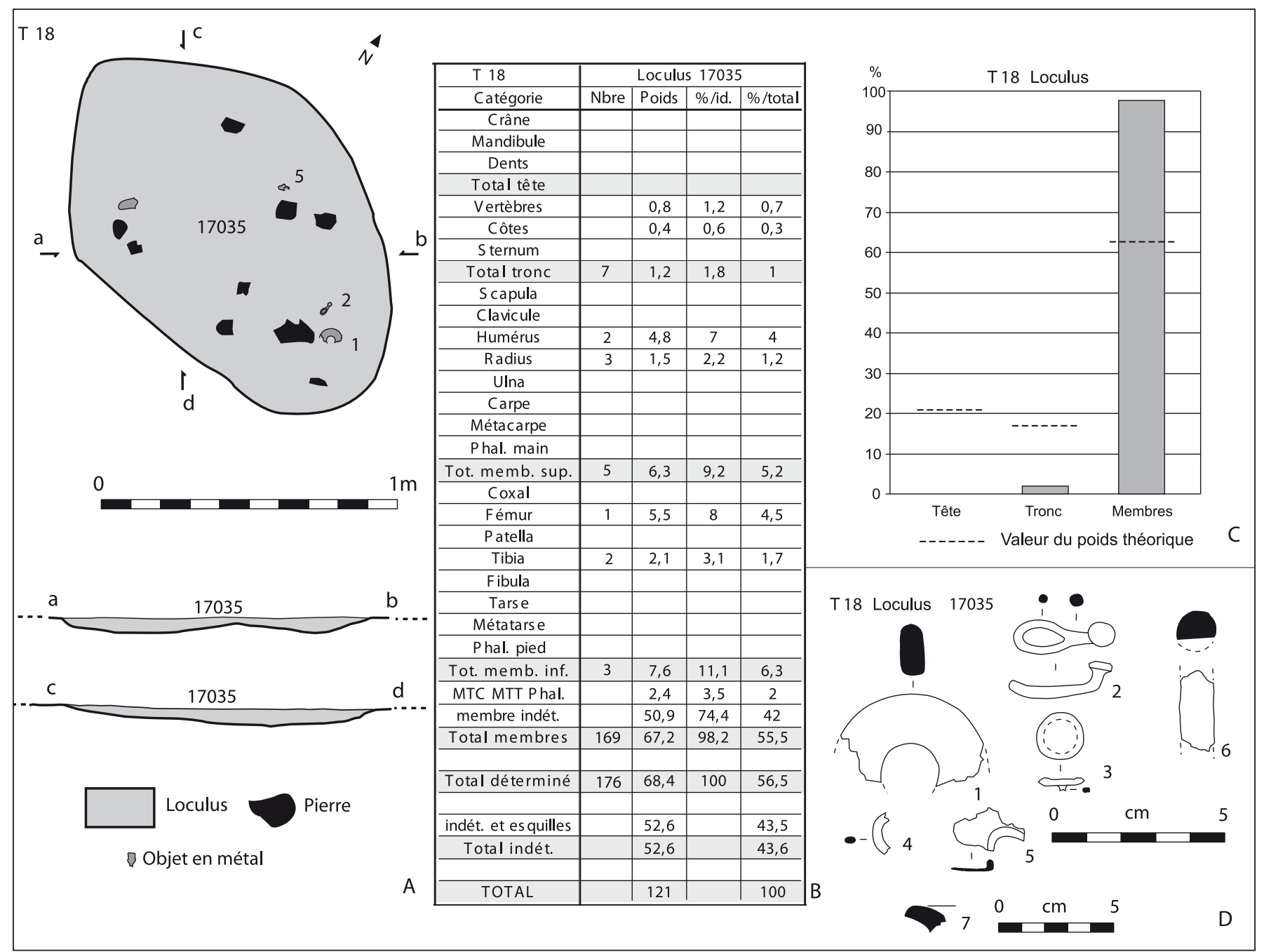

Fig. 27. Tombe T18. A : plan et coupes. B : tableau de détermination des os humains (poids en grammes ; calcul des \% sur la masse). $\mathrm{C}$ : répartition du poids des os par grandes régions anatomiques (\% par rapport au total déterminé).

$D:$ mobilier ( 1,3 et $6:$ fer $; 2,4$ et $5:$ bronze $; 7:$ céramique non tournée).

centrale ; départ de la tige de fixation, de section quadrangulaire, très fine (l'extrémité manque); diam.: $13 \mathrm{~mm}$ (fig. 27, C, $\mathrm{n}^{\circ} 3$ ).

- Deux éléments de la ceinture de suspension d'un fourreau d'épée :

- Anneau circulaire plat, en fer (fig. 27, C, $\mathrm{n}^{\circ} 1$ ), fragmentaire; section rectangulaire ; diam.: $45 \mathrm{~mm}$; haut. : $8 \mathrm{~mm}$. Cet objet est passé sur le bûcher ;

- Agrafe de ceinture en bronze; boucle ovalaire; crochet relevé à angle droit, terminé par un bouton circulaire ; long. : $30 \mathrm{~mm}$; larg. : $11 \mathrm{~mm}$. Cet objet est passé sur le bûcher (fig. 27, C, $\mathrm{n}^{\circ} 2$ ).

- Moitié de maillon de chaînette en bronze, de section lenticulaire; diam. : $12 \mathrm{~mm}$ (fig. 27, C, $\mathrm{n}^{\circ} 4$ ).

Objets métalliques non déterminés :

- Morceau de plaque en bronze présentant un orifice circulaire bordé d'un bourrelet (long. cons. : $20 \mathrm{~mm}$ ) et deux autres fragments en bronze, tous partiellement fondus ; objet non déterminé (fig. 27, C, $\mathrm{n}^{\circ} 5$ ) ;

- Segment de tige en fer; section probablement circulaire; long. cons. : $23 \mathrm{~mm}$; diam. env. $12 \mathrm{~mm}$ (fig. 27, C, $\mathrm{n}^{\circ} 6$ ).

Tessons de vases isolés :

- 2 petits tessons d'une urne non tournée: bord déversé à lèvre aplatie ; diam. ouv. : 140 mm (fig. 27, $\left.\mathrm{C}, \mathrm{n}^{\circ} 7\right)$;

- 21 petits tessons de panses d'urnes non tournées, appartenant semble-t-il, à 20 exemplaires différents (non dessinés).

Os d'animaux (détermination A. Gardeisen) :

- Capriné : 1 fragment de molaire supérieure ;

- Indéterminés : 4 esquilles brûlées, noires ;

- Microfaune : 2 fragments. 


\section{La tombe T19}

\section{1. État et composantes de la tombe}

La tombe T19 abrite un seul sujet incinéré, un adulte. Le sexe ne peut être déterminé, mais, d'après la symbolique du mobilier, la nature et l'abondance des objets de parure, il semble cependant s'agir d'une femme. La sépulture comprend un loculus qui a pu être entièrement fouillé mais aucune trace d'une éventuelle structure de recouvrement n'a été décelée (fig. 28, A, B et C).

\subsection{Le défunt}

Le défunt est représenté par 437,7 g d'os brûlés. D'après la fermeture des apex des dents, la morphologie des os, et en particulier la soudure du listel sur le corps vertébral, on a affaire à un adulte. Il pourrait en fait s'agir d'un adulte de plus de trente ans, car les sutures, souvent colmatées en face endocrânienne, sont toujours libres en face exocrânienne. Le sexe anthropologique ne peut pas être précisé (fig. 28, D).

Le poids d'os déposés représente $27 \%$ du poids moyen d'un squelette d'adulte brûlé, $1627,1 \mathrm{~g}$ selon McKinley (1993). Le taux d'identification des fragments est de $68,5 \%$ du poids total. Avec $21,1 \%$ du poids identifié, la représentation du crâne est conforme au poids théorique $(20,4 \%)$. Celle du tronc est faible, $8,7 \%$ (poids théorique: $17 \%$ ), tandis que les membres sont sur-représentés, 70,2\% du poids identifié au lieu des $62,6 \%$ attendus (fig. 28, E). La couleur blanc crayeux révèle une température de crémation supérieure à $650^{\circ}$ selon le barème de E. Bonnucci et G. Grazziani (1975), et les morceaux de diaphyses sont très souvent fissurés en ondes concentriques, indice d'une incinération d'os frais.

Les os ont été placés dans le loculus, hors de tout contenant. Ils sont dispersés, mais leur densité est cependant plus forte dans la partie centrale de la structure.

Le poids moyen des fragments déterminés, toutes régions anatomiques confondues, est de $0,32 \mathrm{~g}(0,27 \mathrm{~g}$ pour la tête, $0,30 \mathrm{~g}$ pour le tronc et $0,34 \mathrm{~g}$ pour les membres). Pour les 25 fragments identifiés comme provenant des membres supérieurs, le poids moyen est de $1,34 \mathrm{~g}$; pour les 8 fragments identifiés comme provenant des membres inférieurs, il est de 1,39 $\mathrm{g}$.

\subsection{Le loculus}

Le loculus est une large cuvette, de plan ovalaire, de $1,45 \mathrm{~m}$ et $1,25 \mathrm{~m}$ sur ses axes. Sa profondeur est faible en regard de son ouverture, $13 \mathrm{~cm}$ au maximum, et son fond est irrégulier (fig. 28, A, B et C). Le comblement est un sédiment limoneux gris foncé à noirâtre. Cette couleur n'est cependant pas homogène, la partie centrale étant plus sombre que la périphérie. Ce sédiment renferme, dispersées, quelques rares pierres calcaires non roulées, ne dépassant pas $10 \mathrm{~cm}$ de côté, certaines présentant des éclatements dus au feu. On y trouve également de petits nodules d'argile plus ou moins cuite, ainsi que, surtout dans la partie centrale, de petits charbons de bois (moins de $5 \mathrm{~mm}$ ). Le matériel céramique est rare, en tout 46 petits tessons épars, se rapportant à autant de vases différents ; en revanche le mobilier de bronze, également dispersé en plan et en hauteur, est abondant. Nombre de fragments de bronze présentent un début de fusion.

\section{Mobilier du loculus}

Objets de parure et d'habillement :

- Un pendant d'oreille en argent, en forme de croissant renflé en sa partie centrale et s'amincissant vers les deux extrémités pointues qui se chevauchent légèrement; diam. : 12,8 à 13,3 mm (fig. 29, $\left.\mathrm{n}^{\circ} 1\right)$;

- Une perle en pâte de verre bleue, en cours de fusion ; diam. : 11 à $12 \mathrm{~mm}$; ép. : env. $7 \mathrm{~mm}$ (fig. 29, $\left.\mathrm{n}^{\circ} 2\right)$;

- Un bracelet ou un anneau de cheville en bronze moulé, ouvert, formé d'une succession d'oves pleins et peu proéminents, reliés l'un à l'autre par une moulure, chacune des deux extrémités étant constituée par un de ces oves. Cet objet a été tordu et cassé en plusieurs morceaux, ce qui ne permet pas de calculer précisément le diamètre et donc, de savoir s'il été porté au bras ou à la cheville. Long. déroulée : $155 \mathrm{~mm}$; haut de la tige : $18 \mathrm{~mm}$. (fig. 29, $\mathrm{n}^{\circ} 3$ );

- Fragments d'une fibule: morceau de ressort en bronze, de 3 spires conservées (diam. : $9 \mathrm{~mm}$ ); quatre petits éléments de corail : trois triangulaires ou trapézoïdaux, moulurés et perforés d'un petit trou de fixation ; un en arc de cercle, orné sur une face de petits godrons. Malgré le caractère très incomplet de cet objet, on peut y reconnaître une fibule en bronze, à ressort bilatéral relativement long, pourvue d'un pied replié vers l'arc et terminé par un disque supportant la décoration de corail, triangles et losanges rayonnants et arc périphérique (type 11 de C. Tendille). Les éléments de corail ont subi l'action du feu et sont de couleur grise (fig. 29, $\mathrm{n}^{\circ} 4$ ) ;

- Nombreux éléments d'une ceinture en bronze, dite "féminine" de type méridional, comprenant une chaîne 


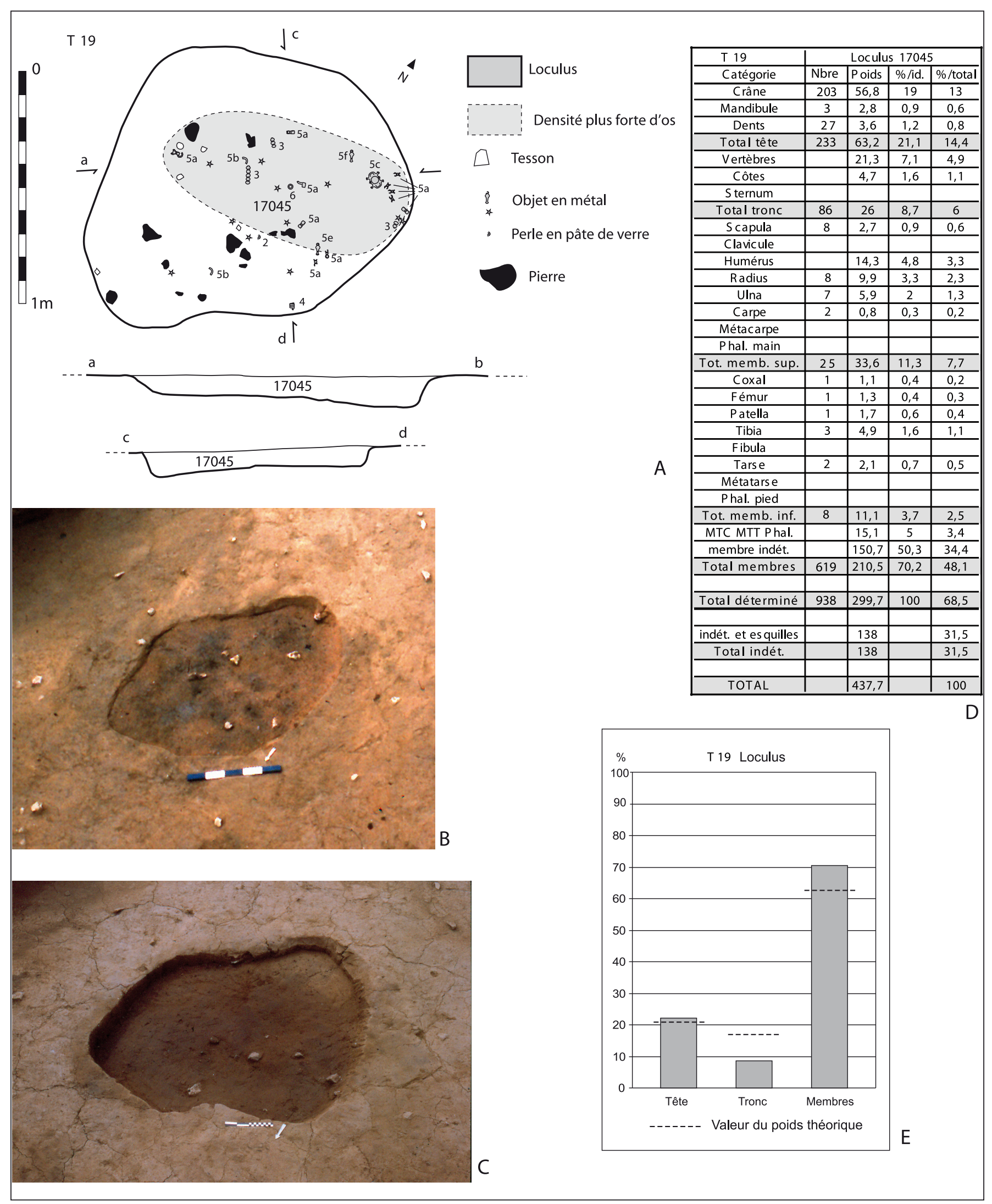

Fig. 28. Tombe T19. A : plan et coupes. B : remplissage du loculus en cours de fouille, vu du nord. C : loculus en fin de fouille, vu du nord. $\mathrm{D}$ : tableau de détermination des os humains (poids en grammes; calcul des $\%$ sur la masse). E : répartition du poids des os par grandes régions anatomiques (\% par rapport au total déterminé). 


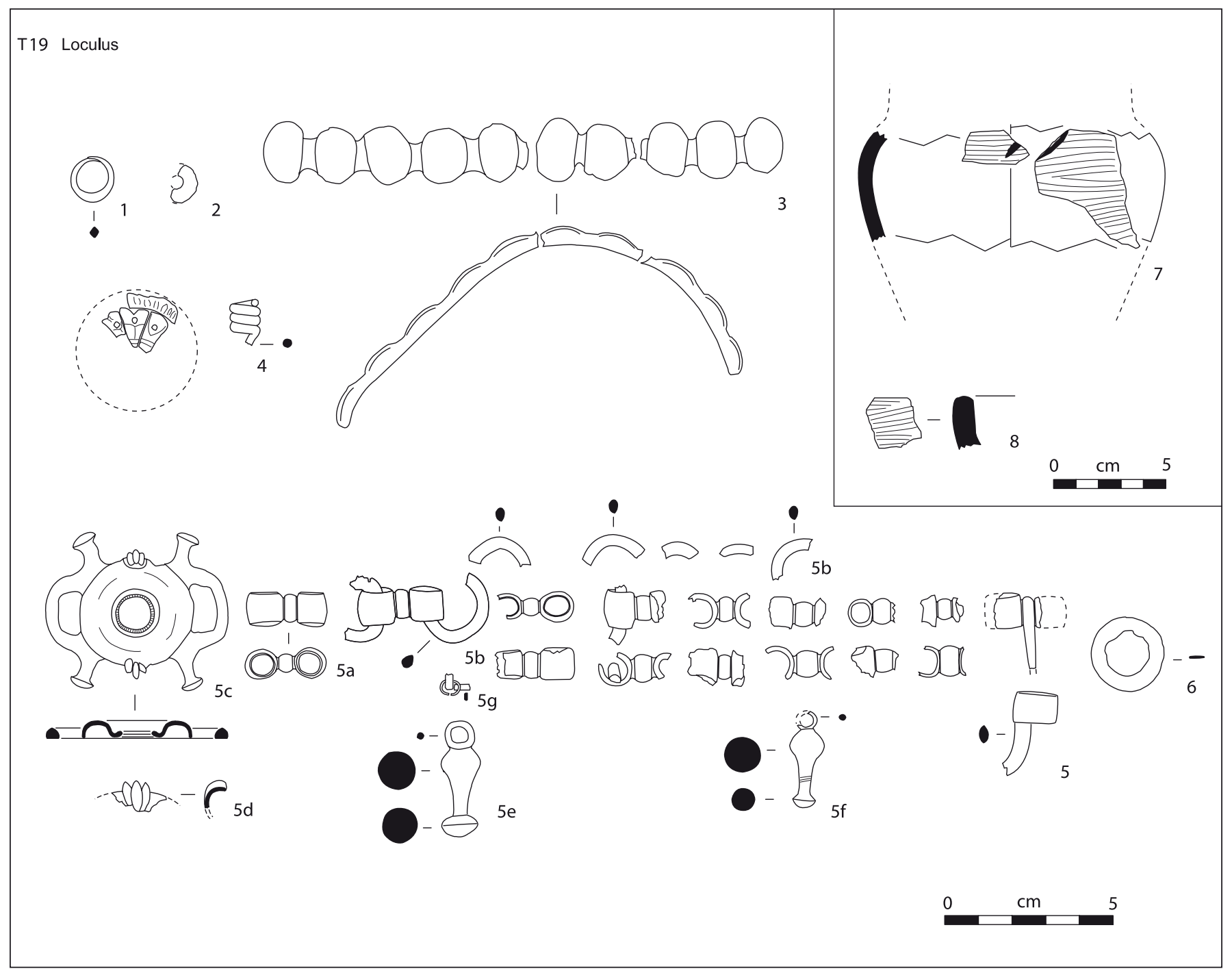

Fig. 29. Tombe T19. Mobilier ( $1:$ argent ; $2:$ verre ; $4:$ fibule en bronze et corail ; 3,5 et $6:$ bronze ; 7 et $8:$ céramique non tournée).

composite à laquelle s'accrochent des pendentifs et des agrafes, soit les éléments suivants (fig. 29, $\mathrm{n}^{\circ} 5$ et fig. 115) :

- 10 à 12 maillons doubles, complets ou fragmentaires ; les deux anneaux, en ruban plat, sont reliés par une courte tige à moulure simple; Long. des exemplaires complets : 24 à $25 \mathrm{~mm}$; larg. maillons : 9 à $10 \mathrm{~mm}$; larg. moulures : 7 à 8 mm (fig. 29, $\left.n^{\circ} 5 a\right)$;

- 4 ou 5 anneaux simples, fragmentaires, de section lenticulaire, reliant les maillons doubles entre eux ; diam. : $10 \mathrm{~mm}$; haut. : $3 \mathrm{~mm}$ (fig. 29, $\mathrm{n}^{\circ} 5 \mathrm{~b}$ ) ;

- une agrafe circulaire ajourée, formée d'un anneau de section en «U», portant deux appendices ajourés diamétralement opposés, terminés chacun par deux boutons en calotte sphérique ; entre les appendices, prend place une triple moulure sur le bord de l'anneau ; Long. : $55 \mathrm{~mm}$; haut. $\max .: 47 \mathrm{~mm}\left(\right.$ fig. $\left.29, \mathrm{n}^{\circ} 5 \mathrm{c}\right)$;
- morceau d'une seconde agrafe du même type que la précédente ; il n'en reste que la mouluration ornant le bord de l'anneau entre les appendices (fig. 29, $\mathrm{n}^{\circ} 5 \mathrm{~d}$ ) ;

- 2 pendentifs coniques, renflés, munis d'un anneau de suspension, terminés par un bouton en calotte sphérique ; Long. : respectivement 34 et $28 \mathrm{~mm}$ (fig. 29, $\mathrm{n}^{\circ} 5 \mathrm{e}$ et $\left.5 \mathrm{f}\right)$.

- un morceau de chaînette ; petits maillons ouverts, formés d'un petit ruban plat, recourbé, aux extrémités jointives ; diam. : $6 \mathrm{~mm}$ (fig. 29, $\mathrm{n}^{\circ} 5 \mathrm{~g}$ ).

Certains éléments de cette parure ont subi un début de fusion.

- Un anneau en bronze, très plat, aux bords amincis presque affûté à l'extérieur ; deux irrégularités sur le pourtour intérieur peuvent correspondre à des traces d'usure ; diam. : $21 \mathrm{~mm}$; larg. : $4 \mathrm{~mm}$; ép. : 1,5 mm (fig. 29, $n^{\circ} 6$ ). Cet objet peut appartenir à la ceinture $n^{\circ} 1$. 


\section{Matériel céramique}

Portions de vases :

- Petite portion d'une urne non tournée de forme probable CNT-LOR U5d, 8 tessons représentant moins de $5 \%$ du récipient : panse ovoïde peignée horizontalement, épaule décorée de grandes impressions obliques au peigne, $\left(\right.$ fig. $\left.29, \mathrm{n}^{\circ} 7\right)$;

- Petite portion d'une jatte non tournée de forme CNT-LOR J1a, 17 tessons représentant moins de $5 \%$ du récipient : panse à profil légèrement arrondie, peignée à l'extérieur, bord évasé à lèvre arrondie (fig. 29, n $^{\circ}$ ).

Tessons de vases isolés :

- 1 petit fragment informe de vase à vernis noir de l'atelier de Rosas ;

-1 petit tesson de panse d'amphore à pâte jaune (ibérique ?) ;

-20 petits tessons informes et esquilles de vases non tournés, indéterminés, appartenant semble-t-il, à autant de récipients.

Os d'animaux (détermination A. Gardeisen) :

- Capriné : 1 fragment crânial d'axis, brûlé ;

- 3 fragments de côtes et 1 fragment crânien, brûlés à

blanc, d'animaux de petite taille ;

- 1 fragment de diaphyse d'os long d'animal de grande taille ;

- Indéterminés : 1 esquille non brûlée, 1 esquille brûlée à blanc et 23 esquilles brûlées, noires.

\section{La tombe T20}

\section{1. État et composantes de la tombe}

La connaissance de cette tombe, qui se rapporte à un individu adulte de sexe non déterminable, est probablement très lacunaire. Seule a été fouillée une portion d'une grande lentille d'alluvions enrobant du matériel (US 17046), située en limite du secteur accessible de la nécropole. Cette structure peut donc se prolonger vers le sud. Sa partie fouillée repose directement sur le paléosol de la nécropole et aucun loculus n'a été repéré au-dessous. Il est possible que cet épandage constitue une tombe à part entière, mais, vu la morphologie des autres sépultures fouillées alentours, il pourrait plutôt s'agir d'une structure de recouvrement d'une tombe dont le loculus se situerait plus au sud, hors du secteur fouillé (fig. 30, A et B).

\subsection{Le défunt}

Cet épandage (US 17046) a livré 23,1 g d'os humains brûlés, dispersés (fig. 30, C). Ils appartiennent à un adulte : un fragment de voûte crânienne présente une suture libre en face exocrânienne et en cours de synostose en face endocrânienne. Le sexe n'est pas précisable.

Les os sont très fragmentés et peu d'éléments sont identifiables (56,3\% seulement du poids total d'os). Le crâne représente $16,1 \%$ du poids total d'os déterminés, le tronc $0,8 \%$ et les membres $83,1 \%$ (fig. 30, D).

L'incinération a affecté des os frais, comme le montrent les fissurations en ondes concentriques des diaphyses. Presque tous les restes ont une couleur blanc crayeux, traduisant une température de crémation de l'ordre de $650^{\circ}$ selon le barème de $\mathrm{E}$. Bonnucci et G. Grazziani (Bonucci, Grazziani 1975).

Le poids moyen des fragments déterminés, toutes régions anatomiques confondues, est de $0,13 \mathrm{~g}(0,11 \mathrm{~g}$ pour la tête, $0,03 \mathrm{~g}$ pour le tronc et $0,14 \mathrm{~g}$ pour les membres).

\subsection{L'aire d'épandage}

Le matériel recueilli se répartit sur une aire d'au moins $1,1 \mathrm{~m}$ de long (mais une prolongation au sud hors du secteur fouillé ne peut être écartée) sur 0,6 m de large, à la surface du paléosol de la nécropole. Cette aire est composée d'alluvions limoneuses brunâtres semblables à celles qui recouvrent le sol, et ne s'en différencie que par la présence de petits fragments d'os humains brûlés, quelques petites pierres calcaires non roulées ayant éclaté au feu (aucune ne dépasse $10 \mathrm{~cm}$ d'arête), de rares petits charbons de bois épars, ainsi que quelques tessons de vases non tournés.

\section{Mobilier de l'aire d'épandage}

- Portion de la partie inférieure d'un grand couvercle non tourné de forme probable CNT-LOR V2a, 2 tessons représentant environ $20 \%$ du vase ; paroi, semble-t-il, rectiligne, pied annulaire, fond percé d'un large trou après cuisson; diam. pied : $70 \mathrm{~mm}\left(\right.$ fig. $\left.\mathbf{3 0}, \mathrm{E}, \mathrm{n}^{\circ} 1\right)$;

- 9 petits tessons de céramiques non tournées appartenant, semble-t-il, à autant de récipients.

Os d'animaux (détermination A. Gardeisen) :

-1 fragment de racine de molaire (cf. bœuf) ;

- Indéterminés : 2 esquilles brûlées, noires. 


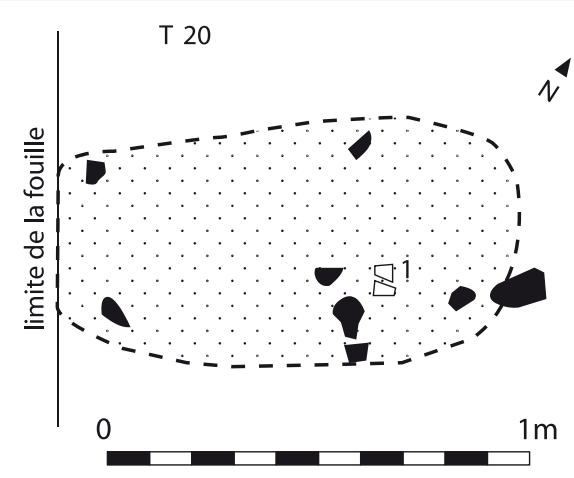

$\because \quad$ Épandage de sédiment 1 Limite hypothétique avec os incinérés 1 du recouvrement
Pierre
$\triangle$ Tesson
A

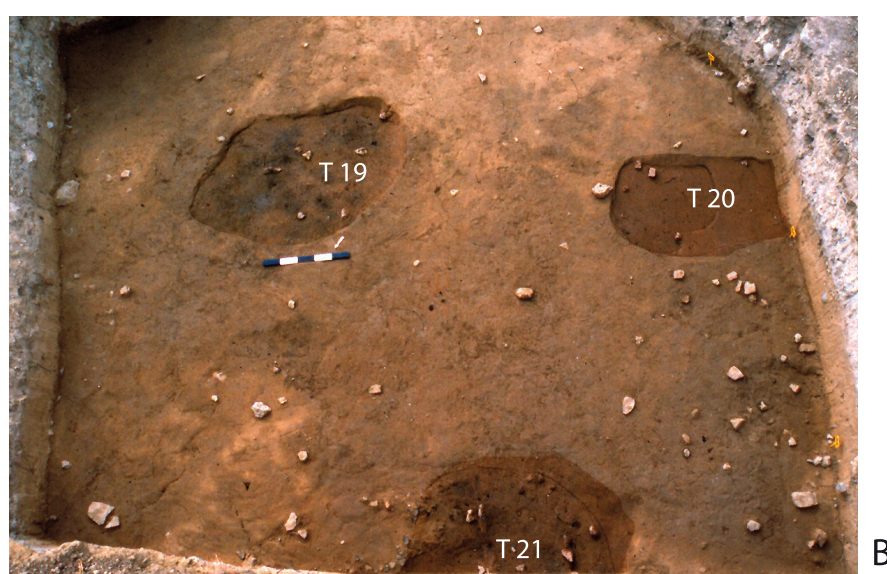

B

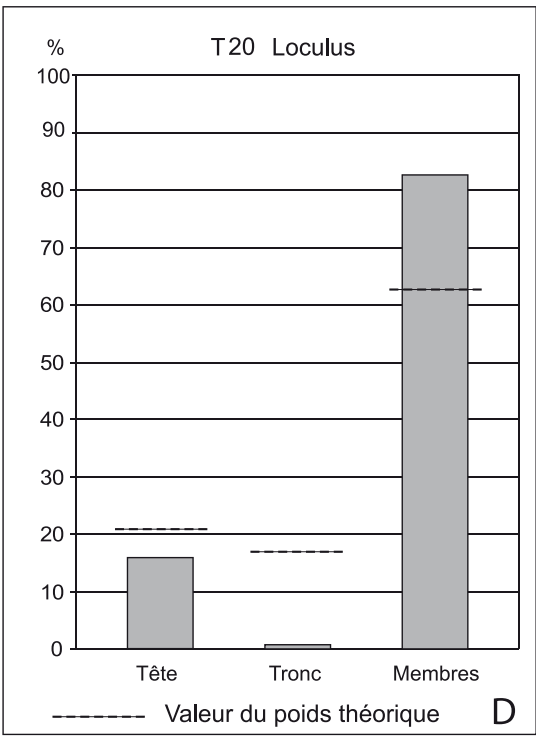

\begin{tabular}{|c|c|c|c|c|}
\hline T 20 & \multicolumn{4}{|c|}{ Loculus 17046} \\
\hline Catégorie & Nbre & Poids & $\% /$ id. & $\% /$ total \\
\hline Crâne & 16 & 1,9 & 14,6 & 8,2 \\
\hline \multicolumn{5}{|l|}{ Mandibule } \\
\hline Dents & 3 & 0,2 & 1,5 & 0,9 \\
\hline Total tête & 19 & 2,1 & 16,1 & 9,1 \\
\hline \multicolumn{5}{|l|}{ Vertèbres } \\
\hline Côtes & & 0,1 & 0,8 & 0,4 \\
\hline \multicolumn{5}{|l|}{ Sternum } \\
\hline Total tronc & 4 & 0,1 & 0,8 & 0,4 \\
\hline \multicolumn{5}{|l|}{ Scapula } \\
\hline \multicolumn{5}{|l|}{ Clavicule } \\
\hline \multicolumn{5}{|l|}{ Humérus } \\
\hline \multicolumn{5}{|l|}{ Radius } \\
\hline \multicolumn{5}{|l|}{ Ulna } \\
\hline \multirow{2}{*}{\multicolumn{5}{|c|}{$\begin{array}{c}\text { Carpe } \\
\text { Métacarpe }\end{array}$}} \\
\hline & & & & \\
\hline \multicolumn{5}{|l|}{ Phal. main } \\
\hline \multicolumn{5}{|l|}{ Tot. memb. sup. } \\
\hline \multirow{2}{*}{\multicolumn{5}{|c|}{$\begin{array}{l}\text { Coxal } \\
\text { Fémur }\end{array}$}} \\
\hline \multirow{2}{*}{\multicolumn{5}{|c|}{$\frac{\text { Fémur }}{\text { Patella }}$}} \\
\hline & & & & \\
\hline Tibia & 1 & 0,6 & 4,6 & 2,6 \\
\hline \multicolumn{5}{|l|}{ Fibula } \\
\hline \multicolumn{5}{|l|}{ Tarse } \\
\hline \multicolumn{5}{|l|}{ Métatarse } \\
\hline \multicolumn{5}{|l|}{ P hal. pied } \\
\hline Tot. memb. inf. & 1 & 0,6 & 4,6 & 2,6 \\
\hline MTC MTT P hal. & & 0,9 & 6,9 & 3,9 \\
\hline membre indét. & & 9,3 & 71,5 & 40,3 \\
\hline Total membres & 77 & 10,8 & 83,1 & 46,8 \\
\hline Total déterminé & 100 & 13 & 100 & 56,3 \\
\hline & & & & \\
\hline Total indét. & & 101 & & 43,7 \\
\hline & & & & \\
\hline TOTAL & & 23,1 & & 100 \\
\hline
\end{tabular}

C
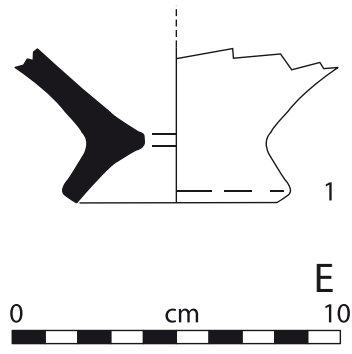

Fig. 30. Tombe T20. A : plan. B : vue des loculus des tombes 19, 20 et 21 prise du nord. C : tableau de détermination des os humains (poids en grammes ; calcul des \% sur la masse). D : répartition du poids des os par grandes régions anatomiques (\% par rapport au total déterminé).

E : mobilier (céramique non tournée). 


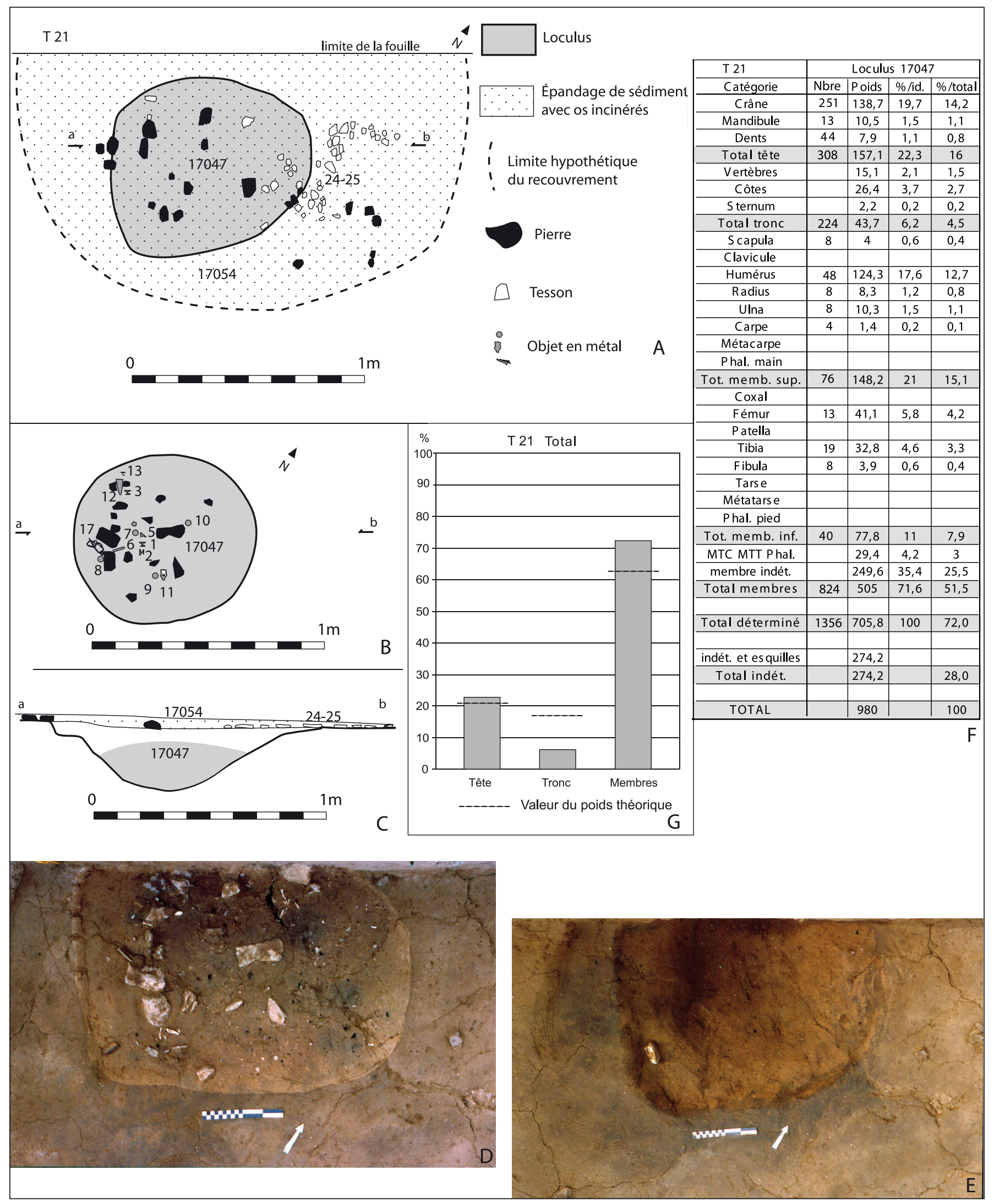

Fig. 31. Tombe 21. A : plan au niveau de la structure de recouvrement. B : plan du remplissage du loculus. C : coupe. D : remplissage du loculus en cours de fouille, vu du sud-est. E : loculus en fin de fouille, vu du sud-est. F : tableau de détermination des os humains (poids en grammes ; calcul des \% sur la masse). G : répartition du poids des os par grandes régions anatomiques (\% par rapport au total déterminé). 


\section{La tombe $\mathrm{T} 21$}

\section{1. État et composantes de la tombe}

La tombe T21 accueille un seul sujet incinéré, un adulte de sexe non déterminable; le mobilier (voir infra) paraît cependant symboliser un homme. La sépulture comprend un loculus et une structure de recouvrement débordant largement tout autour, mais seule la moitié méridionale de cette dernière a pu être entièrement fouillée (fig. 31, A à E).

\subsection{Le défunt}

Le défunt est représenté par $980 \mathrm{~g}$ d'os brûlés (fig. 31, F). La morphologie des os, et en particulier les soudures des extrémités aux corps diaphysaires, ainsi que la fermeture des apex des dents montrent qu'il s'agit d'un adulte. Il pourrait cependant s'agir d'un adulte jeune car la face endocrânienne montre seulement un début de synostose des sutures, tandis que celles-ci sont encore libres sur l'exocrâne. Le sexe anthropologique ne peut pas être précisé. La couleur blanc crayeux révèle une température de crémation supérieure à $650^{\circ}$ selon le barème de E. Bonnucci et G. Grazziani (1975), et les morceaux de diaphyses sont très souvent fissurés en ondes concentriques, indice d'une incinération d'os frais.

Ces os ont été placés dans le loculus, hors de tout contenant, mais le mode de dépôt traduit deux gestes successifs. Dans la partie inférieure et centrale du loculus, a été déposé un magma cendreux et charbonneux, gris foncé à noirâtre, gluant, contenant de très nombreux morceaux d'os, souvent amalgamés entre eux, parfois de grande taille, certains segments de diaphyses atteignant quatre centimètres de long. Dans la partie périphérique du comblement, de couleur grisâtre à marron, les restes osseux, moins nombreux et plus fragmentés, sont dispersés. Aucune incompatibilité n'existe entre les deux lots qui, par ailleurs, proviennent des mêmes régions du corps. La partie fouillée du recouvrement n'a pas livré d'os humains ; on ne peut affirmer toutefois que la totalité des restes osseux ait été déposée dans le loculus, car on ignore si la moitié non fouillée du recouvrement est à l'image de la partie sud.

Le poids d'os déposés dans le loculus représente $60 \%$ du poids moyen d'un squelette d'adulte brûlé, 1627,1 g selon McKinley (1993). Les taux d'identification sont de $72 \%$ du poids total des fragments. La sur-représentation des membres est importante, 71,6\% du poids identifié pour un poids théorique de $62,6 \%$; le crâne est très légèrement sur-représenté, $22,3 \%$ au lieu des $20,4 \%$ attendus ; la représentation du tronc reste très faible, 6,2 \% (poids théorique : $17 \%$ ) (fig. 31, G).

Le poids moyen des fragments déterminés, toutes régions anatomiques confondues, est de $0,52 \mathrm{~g}(0,51 \mathrm{~g}$ pour la tête, $0,20 \mathrm{~g}$ pour le tronc et $0,61 \mathrm{~g}$ pour les membres). Le poids moyen est de $1,95 \mathrm{~g}$ pour les 76 fragments identifiés comme provenant des membres supérieurs et pour les 40 fragments identifiés comme provenant des membres inférieurs.

\subsection{Le loculus}

Le loculus (US 17047) est une cuvette de plan ovale de 70 à $90 \mathrm{~cm}$ d'axes à l'ouverture, à fond arrondi et aux parois abruptes, profonde de $31 \mathrm{~cm}$ (fig. 31, A à E). Le remplissage est composé d'un sédiment limoneux gris foncé à noirâtre, gluant, vers le centre et vers le fond, grisâtre à marron vers la périphérie. Il inclut, épars dans sa zone centrale comme dans sa périphérie, des nodules d'argile chauffée, des charbons de bois, en général inférieurs à $0,5 \mathrm{~cm}$, de rares pierres calcaires non roulées, n'excédant pas $10 \mathrm{~cm}$ d'arête, mais le plus souvent plus petites, certaines ayant éclaté au feu, des os humains brûlés, et des objets dispersés dans toute l'épaisseur du remplissage: trois tessons seulement de deux récipients, mais un abondant mobilier métallique se rapportant à une épée $\left(\mathrm{n}^{\circ} 1\right.$ à 5$)$, son fourreau $\left(\mathrm{n}^{\circ} 6\right)$ et sa suspension $\left(\mathrm{n}^{\circ} 7\right.$ à 11$)$, une lance $\left(\mathrm{n}^{\circ} 12\right)$, un bouclier $\left(\mathrm{n}^{\circ} 13\right.$ à $16)$, à $1^{\prime}$ habillement $\left(n^{\circ} 17\right)$ et à trois objets non déterminés ( $\mathrm{n}^{\circ} 18$ à 23 ). Certains des objets en bronze sont en fusion.

\section{Mobilier du loculus}

Objets personnels : armement et fourniment

- Cinq rivets en fer (fig. 32, $\mathrm{n}^{\circ} 1$ à 5), pourvus d'une tête discoïde à chacune des deux extrémités, permettant la fixation des éléments de garniture de la poignée d'une épée ; l'un d'eux ( $\left.{ }^{\circ} 5\right)$ est cassé au niveau du départ d'une tête. Les têtes ont un même diamètre de chaque côté, mais, selon l'extrémité, elles n'ont cependant pas la même forme : légèrement bombées d'un côté ; de l'autre légèrement concaves (fig. 32, $\mathrm{n}^{\circ} 1$ - 3 et sans doute 5) ou avec un petit bourrelet périphérique $\left(\mathrm{n}^{\circ} 4\right)$; les tiges ont une section ronde ; long. $\mathrm{n}^{\circ} 1$ à $4: 20 \mathrm{~mm} ; 20 \mathrm{~mm} ; 18 \mathrm{~mm}$; $24 \mathrm{~mm}$; long. cons. $\mathrm{n}^{\circ} 5: 18 \mathrm{~mm}$; diam. têtes : $11 \mathrm{~mm}$;

- Six morceaux de bord en gouttière de la plaque avers d'un fourreau d'épée, en tôle de fer (fig. 32, 


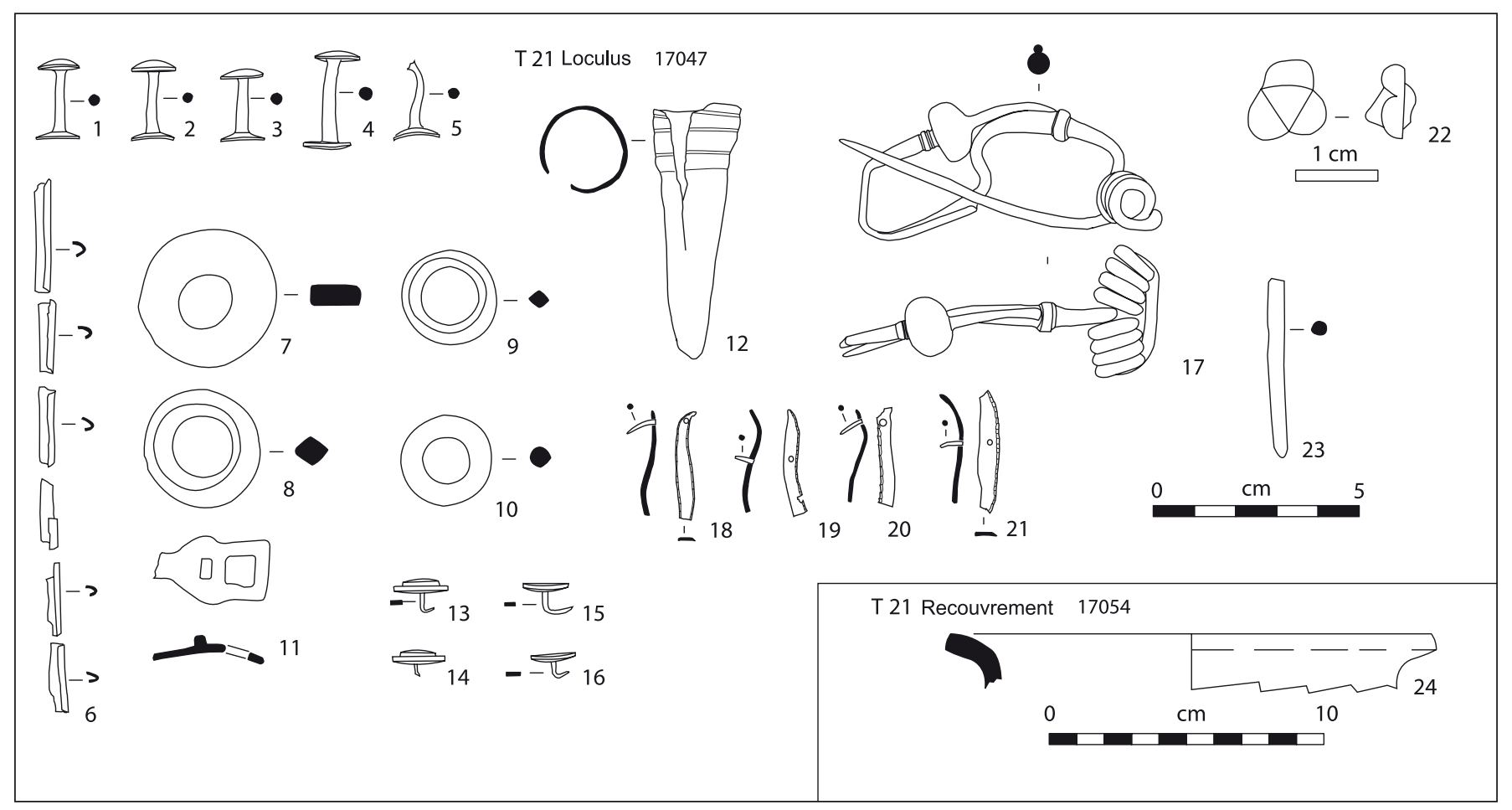

Fig. 32. Tombe T21. Mobilier (1-9 et 12-21 et $23:$ fer ; 10, 11 et $22:$ bronze ; $24:$ céramique non tournée).

$\left.\mathrm{n}^{\circ} 6\right)$; long. cons. : $27 \mathrm{~mm} ; 16,5 \mathrm{~mm} ; 18 \mathrm{~mm} ; 16 \mathrm{~mm}$; $16 \mathrm{~mm}$; ép. tôle : $0,7 \mathrm{~mm}$;

- Quatre anneaux (fig. 32, no 7 à 10), dissemblables, en fer ou en bronze, et une agrafe en bronze $\left(n^{\circ} 11\right)$, se rapportant à la lanière de cuir permettant la suspension d'un fourreau d'épée :

- Anneau circulaire plat, en fer (fig. 32, $\mathrm{n}^{\circ}$ 7), section rectangulaire; légèrement bombé sur une face; diam. : $35 \mathrm{~mm}$; haut. : 4,5 à $8 \mathrm{~mm}$;

- Anneau circulaire, en fer (fig. 32, $\mathrm{n}^{\circ} 8$ ), section losangique ; diam. : $29 \mathrm{~mm}$; haut. : $7 \mathrm{~mm}$;

- Anneau circulaire, en fer (fig. 32, $\mathrm{n}^{\circ}$ 9), section losangique; diam. : 23 à $24 \mathrm{~mm}$; haut. : $5 \mathrm{~mm}$;

- Anneau circulaire, en bronze (fig. 32, $\mathrm{n}^{\circ}$ 10), section circulaire ; diam. : 22,5 mm ; haut. : $5 \mathrm{~mm}$;

- Agrafe de ceinture, en bronze (fig. 32, $\mathrm{n}^{\circ} 11$ ); boucle rectangulaire ; corps cordiforme ; crochet manquant ; long. cons. : $27 \mathrm{~mm}$; larg. : $16 \mathrm{~mm}$.

- Talon de lance en fer $\left(\mathrm{n}^{\circ} 12\right)$, formé d'une simple douille conique, courte, large et à pointe mousse, élaborée à partir d'une feuille de tôle enroulée dont les bords sont bien visibles; trois moulures vers le bord; pas de trou de fixation apparent; diam. ouv. : 21 à $21,5 \mathrm{~mm}$; long. : $62 \mathrm{~mm}$; ép. $1 \mathrm{~mm}$.

- Quatre rivets de fixation d'ailettes d'un umbo de bouclier, en fer (fig. 32, n 13 à 16) :

- deux exemplaires à tête discoïde (fig. 32, $\mathrm{n}^{\circ} 13$ et 14), dont la partie centrale est légèrement bombée ; diam. : $13 \mathrm{~mm}$; tige de section quadrangulaire très fine, incomplète pour le $n^{\circ} 14$, repliée à angle droit pour le $\mathrm{n}^{\circ} 13$ et déterminant une épaisseur de $4 \mathrm{~mm}$ pour la planche du bouclier ;

- deux exemplaires à tête discoïde (fig. 32, n 15 et 16), dont la partie centrale est légèrement concave ; diam. : $11 \mathrm{~mm}$; tige de section quadrangulaire très fine, repliée à angle droit et déterminant une épaisseur de $4 \mathrm{~mm}$ et de $3 \mathrm{~mm}$ pour la planche du bouclier.

Objet personnel : parure et habillement

- Fibule en fer (fig. 32, $\mathrm{n}^{\circ} 17$ et fig. 115), complète ; ressort à deux fois quatre spires et corde externe ; arc en tige légèrement épaissie au centre, de section circulaire et de forme outrepassée ; grand pied recourbé formant deux angles arrondis et venant se fixer au sommet de l'arc par deux petites griffes; près de l'arc, ce pied est orné d'une grosse perle en bulbe d'oignon, confectionnée dans la masse du métal, précédée de stries transversales ; long porte-ardillon; long. : $77 \mathrm{~mm}$; larg. au ressort : $36 \mathrm{~mm}$; type 13b de R. Gebhard (1991).

Objets métalliques non déterminés :

- Quatre lamelles en fer (fig. 32, $n^{\circ} 18$ à 21), en ruban légèrement sinueux et ondulé, pointu à une extrémité, coupé en biais à l'autre ; les bords sont chanfreinés et décorés de légères indentations ; chaque lamelle possède au moins un fin rivet rectiligne et très pointu ; long. 
lamelle : 25 à $29 \mathrm{~mm}$; larg. max. : $5 \mathrm{~mm}$; ép. : $1 \mathrm{~mm}$; long. max. rivet: $6 \mathrm{~mm}$. Ces lamelles devaient être clouées sur un support; il peut s'agir d'éléments de décoration d'une poignée d'épée, d'un fourreau ou d'un coffret ;

- Tête de clou (?) en bronze ; sur une face, une pointe centrale pyramidale à base triangulaire équilatérale et trois renflements périphériques en croissant; face opposée irrégulière ; $9 \mathrm{~mm}$ d'axes et $7 \mathrm{~mm}$ d'épaisseur maximum (fig. 32, $\mathrm{n}^{\circ} 22$ ) ;

- Segment de tige en fer (fig. 32, $\mathrm{n}^{\circ} 23$ ); long. cons. : $44 \mathrm{~mm}$; diam. : $4 \mathrm{~mm}$ (objet non restauré);

-3 petits fragments de tôle de bronze, recourbée; ép. : $1 \mathrm{~mm}$; objet indéterminé (non dessiné) ;

-5 petits fragments et gouttes de bronze en fusion (non dessiné).

Matériel céramique : tessons de vases isolés

-2 tessons de la panse peignée d'une grande urne non tournée (non dessiné) ;

- 1 tesson de panse d'urne ou d'œnochoé à pâte claire massaliète (non dessiné).

Os d'animaux (détermination A. Gardeisen) :

- 24 fragments d'un métacarpien droit d'équidé, incomplet, se rapportant à l'extrémité proximale, à la diaphyse et à l'extrémité distale (DT proximal: 44,5 mm ; DAP proximal : 29,8 mm). Cet os est brûlé de façon irrégulière, certaines zones étant carbonisées alors que d'autres ne montrent que des effets de la chaleur. Il semblerait qu'il ait été brûlé et en partie cassé sur le feu, à la suite de quoi seuls quelques fragments ont été pris, intentionnellement ou non, pour être déposés dans le loculus. Il paraît en effet peu probable que l'os ait été placé complet dans la tombe : les conditions taphonomiques n'expliqueraient pas la quasi -absence de la diaphyse qui par nature, est une portion extrêmement résistante chez cette espèce ;

- Indéterminés : 23 esquilles non brûlées.

\subsection{La structure de recouvrement}

La partie fouillée de la superstructure US 17054 comprend deux sortes de vestiges. Quelques rares pierres et tessons sont éparpillés, à plat, sur le dessus du comblement du loculus ou sur le paléosol de la nécropole jusqu'à environ $0,5 \mathrm{~m}$ du bord du loculus. Une portion très fragmentée de la partie supérieure d'une urne non tournée est écrasée sur le dessus de la partie orientale du remplissage du loculus et sur le paléosol (fig. 32, $\mathrm{n}^{\circ} 24$ ). L'ensemble est recouvert, sur une épaisseur de 3 à $5 \mathrm{~cm}$ par un épandage d'alluvions limoneuses brunâtres, enfermant de très rares petits fragments d'os humains brûlés. Ce recouvrement déborde de 20 à $50 \mathrm{~cm}$ le loculus, et dans le secteur fouillé mesure $2 \mathrm{~m}$ de diamètre (fig. 31, A).

\section{Mobilier de la structure de recouvrement}

Matériel céramique :

- Portion de la partie supérieure d'une urne non tournée de forme probable CNT-LOR U5d, 120 petits tessons représentant environ $15 \%$ du récipient : bord déversé à lèvre aplatie, col tronconique rentrant lissé, panse ovoïde peignée ; diam. ouv. : $180 \mathrm{~mm}$ (fig. 32, $\mathrm{n}^{\circ} 24$ ) ;

-2 tessons de panse de deux autres vases non tournés (non dessiné).

\section{La tombe T22}

\section{1. État et composantes de la tombe}

La tombe T22 appartient à un jeune enfant, âgé entre deux et quatre ans. Elle comprend un loculus (US 17138) et une structure de surface (US 17136) qui recouvre aussi la tombe 23 toute proche, dont le loculus n'est qu'à $0,4 \mathrm{~m}$ de celui de la tombe 22 . L'ensemble a été entièrement fouillé (fig. 33, $\mathbf{3 4}$ et 35, A et B).

\subsection{Le défunt}

Le défunt est représenté par 88,9 g d'os brûlés (fig. 35, C). L'essentiel (87,4 g) provient du loculus (US 17138); quelques esquilles compatibles avec ce lot, d'un poids de $1,5 \mathrm{~g}$, figurent aussi dans la structure de recouvrement (US 17136). La morphologie des os, particulièrement ceux du crâne, et surtout la dentition permettent de fixer l'âge au décès entre deux et quatre ans : 8 racines et 28 fragments de couronnes de dents déciduales, germes ou fragments de germes de dents définitives dont $\mathrm{I}^{1} \mathrm{~d}$, $\mathrm{I}^{\mathrm{I}} \mathrm{g}, \mathrm{I}_{2} \mathrm{~d}$ ou $\mathrm{g}, \mathrm{C}^{\mathrm{I}} \mathrm{d}$ ou g, $\mathrm{C}_{\mathrm{I}} \mathrm{d}$ ou $\mathrm{g}$, et $\mathrm{M}_{1} \mathrm{~g}$. La couleur blanc crayeux révèle une température de crémation supérieure à $650^{\circ}$ selon le barème de $\mathrm{E}$. Bonnucci et G. Grazziani (1975), et les morceaux de diaphyses sont très souvent fissurés en ondes concentriques, indice d'une incinération d'os frais.

Les taux d'identification sont de $85,3 \%$ du poids total des fragments. La sur-représentation de la tête est très importante, $64,5 \%$ du poids d'os identifiés, mais 


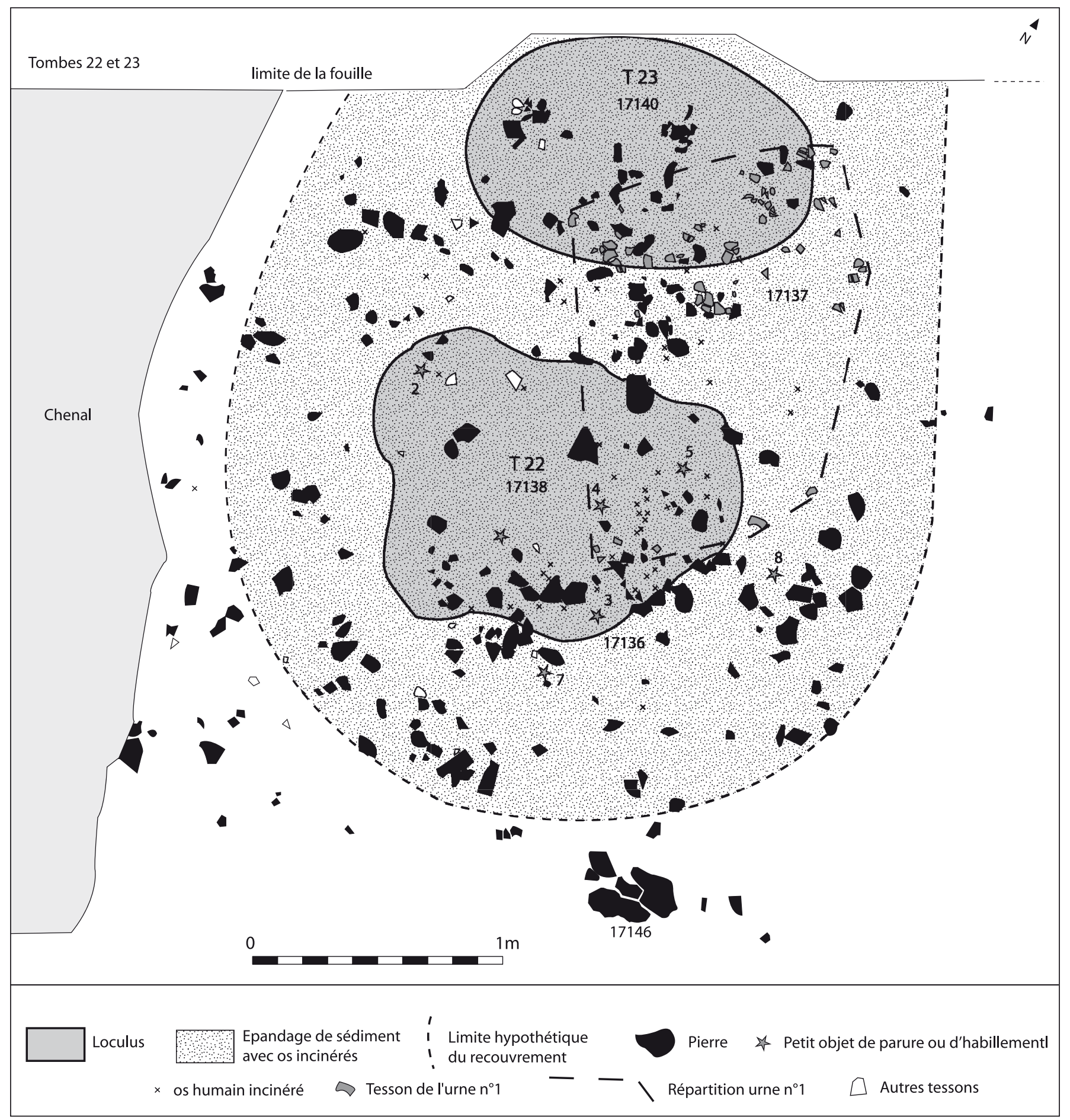

Fig. 33. Recouvrement des tombes T22 et T23 et structure annexe 17146. Plan.

c'est là un fait courant dans les tombes de jeunes enfants (fig. 35, C) (voir supra, tombe 13).

Dans le loculus, les os ont été déposés hors de tout contenant, plus nombreux dans la partie inférieure du comblement (US 17138-2) que dans sa partie supérieure (US 17138-1). Dans la partie inférieure, ont été versées des poignées de résidus du bûcher, non triés : les fragments osseux, de taille souvent supérieure à ceux du haut du loculus, sont agglutinés au sein de plusieurs lentilles cendreuses et charbonneuses. Dans la partie supérieure, les restes du défunt sont éparpillés dans la terre alluviale ayant servi au remplissage de la fosse. Dans la structure de recouvrement, les quelques restes osseux sont dispersés. 


\subsection{Le loculus}

Le loculus (US 17138) est une large cuvette, de plan grossièrement ovalaire, mesurant à l'ouverture $1,5 \mathrm{~m}$ sur l'axe SO-NE et 1,1 m sur l'axe NO-SE. Sa profondeur maximum atteint $36 \mathrm{~cm}$, mais son fond est irrégulier. En effet, son creusement montre deux tranches : une partie supérieure (US 17138-1) large, sur $14 \mathrm{~cm}$ de profondeur, aux parois très peu inclinées ; une partie inférieure (US 17138-2) de moindre extension, de 0,8 m d'axe NS et $0,65 \mathrm{~m}$ d'axe $\mathrm{EO}$, mais de $22 \mathrm{~cm}$ de profondeur, aux parois abruptes (fig. 33, $\mathbf{3 4}$ et $\mathbf{3 5}$, A et B).

Le comblement de la partie supérieure US 17138-1 est composé d'une terre alluviale jaunâtre, par endroits gris sombre, mouchetée de petits charbons de bois millimétriques ou plus rarement centimétriques, épars et, d'une manière générale, ne présentant pas une forte densité. Il comprend des pierres décimétriques, essentiellement réparties, mais de manière discontinue, au-dessus de la partie la plus profonde de la fosse et vers le centre. Ce sont des éléments calcaires non roulés, provenant de la colline du Dévès. Quelques-uns portent des traces nettes de rubéfaction, mais, pour la plupart, il est impossible de savoir si les éclatements sont dus au feu. Sont inclus dans cette partie du comblement, de manière très dispersée, de rares fragments osseux humains incinérés, le plus souvent de très petites dimensions, et de petites perles très fines en pâte de verre bleue.

Le comblement de la partie inférieure US 17138-2 est de couleur plus sombre. C'est toujours une terre limoneuse alluviale, mais le mélange de terre charbonneuse est plus important. Les charbons de bois y sont plus gros et moins rares. Par endroits, on rencontre des poches grises et noirâtres avec morceaux osseux incinérés plus gros mélangés à des charbons et des cendres. De petites perles très fines en pâte de verre bleue, semblables à celles de l'US 17138-1, sont également dispersées dans ce comblement.

De nombreux petits tessons de céramiques non tournées, ainsi que quelques rares tessons de dolium et d'amphore massaliète sont également éparpillés dans le comblement, souvent plaqués contre la paroi de la fosse et en position verticale. Ce loculus est relativement profond et son creusement a atteint et dépassé le niveau d'aménagement caractérisé par des pierres et des galets (us 17125-17180-17184), antérieur à la nécropole et repéré aux alentours immédiats de cette tombe (voir supra chap. 1). La présence de ces tessons de vases dans ce loculus pourrait donc être accidentelle et provenir de cette couche, remaniée lors de l'aménagement du loculus : l'abondance de ces éléments, le fait que chacun, ou presque, relève d'un vase différent et le caractère hétérogène de cet assemblage plaident en faveur de cette hypothèse.

\section{Mobilier du loculus}

Objets de parure :

- Nombreux éléments d'un collier, comprenant :

- Un anneau en bronze, incomplet et brisé en deux morceaux ; section lenticulaire ; diam. ext. : $26 \mathrm{~mm}$; cet objet a subi l'action du feu (fig. 35, E, $\left.\mathrm{n}^{\circ} 1\right)$;

- 56 petites perles en pâte de verre; parmi elles, 55 de couleur bleue et 1 de couleur jaune; section arrondie, fine et irrégulière; diam. ext. : de 5 à $6 \mathrm{~mm}$ pour 55 d'entre-elles, et $7 \mathrm{~mm}$ pour une seule. Beaucoup de ces perles sont plus ou moins déformées par le feu (fig. 35, $\left.\mathrm{E}, \mathrm{n}^{\circ} 2\right)$;

- Une moitié de perle moyenne, en pâte de verre bleue ; section en D ; diam. ext. : 1 mm (fig. 35, E, $n^{\circ} 3$ ) ;

- Une grosse perle, en pâte de verre bleue, complètement déformée par le feu ; diam. ext. : env. 1,5 mm (fig. 35, $\left.\mathrm{E}, \mathrm{n}^{\circ} 4\right)$.

Objet métallique indéterminé :

- Un petit fragment de tôle de bronze découpée; long. cons. : $8 \mathrm{~mm}$; ép. : 0,5 mm (fig. 35, E, nº 5).

Tessons contenus dans le remplissage, dont la présence est peut-être involontaire :

-5 tessons de bords déversés de cinq urnes non tournées, de forme probable CNT-LOR U5d (fig. 35, E, n ${ }^{\circ} 6$ à 10) ;

- 10 tessons d'épaules de quatre urnes non tournées de forme probable CNT-LOR U5d (il peut s'agir des mêmes exemplaires que les précédents) : trois épaules décorées de grandes impressions au peigne disposées en une ou deux lignes brisées (fig. 35, E, $\mathrm{n}^{\circ} 11$ à 13) ; une autre ornée d'impressions au peigne horizontal (fig. 35, E, n 14 ) ; épaules et panses sont peignées horizontalement ;

- 76 petits tessons et quelques esquilles de panses de vases non tournés appartenant, semble-t-il, à autant d'urnes et coupes (non dessinés) ;

- 6 fragments de panses de dolia non tournés (non dessinés) ;

- 6 fragments de panses d'amphores massaliètes (non dessinés) ;

- 1 petit tesson de bord de kylix à pâte claire massaliète de forme CL-MAS 433 (fig. 35, E, n 15);

- 1 morceau de couvercle circulaire, taillé dans un tesson de panse de grand vase non tourné, dépourvu de peignage ; diam. : $85 \mathrm{~mm}$ (fig. 35, $\left.\mathrm{E}, \mathrm{n}^{\circ} 16\right)$. 
Os d'animaux (détermination A. Gardeisen) :

- 4 fragments de frontal d'un porc juvénile, brûlés à blanc ;

- Indéterminés : 49 esquilles à différents degrés de combustion (couleurs bleu, blanc, noir) ;

- Microfaune : 1 os long de batracien.

\section{La tombe $\mathrm{T} 23$}

\section{1. État et composantes de la tombe}

La tombe T23 a accueilli un adulte de sexe non déterminé, mais d'après le mobilier associé, il s'agirait d'une femme. Elle comprend un loculus (US 17140) et une structure de surface (US 17136) qui recouvre aussi la tombe 22. Cette sépulture est complète, à l'exception de la partie septentrionale du recouvrement qui s'étend en dehors de l'emprise de la fouille (fig. 33, 34, et 36, A).

\subsection{Le défunt}

Le défunt est représenté par 352,3 g d'os brûlés, qui proviennent du loculus (US 17140) (fig. 36, B). D'après la morphologie des os, et en particulier la synostose des extrémités aux corps diaphysaires et la fermeture de l'apex sur la racine dentaire, on a affaire à un adulte. Les sutures sont synostosées en face endocrânienne et en cours de synostose en face exocrânienne. Par ailleurs, le sexe anthropologique ne peut être précisé. La crémation a été faite à une température supérieure à $650^{\circ}$ comme le révèle la couleur blanc crayeux des fragments (selon le barème de E. Bonnucci et G. Grazziani ; 1975). Et ce sont bien des os frais qui ont été brûlés car les morceaux de diaphyses sont très souvent fissurés en ondes concentriques. Les os ont été placés dans le loculus, hors de tout contenant, irrégulièrement répartis dans le comblement.

Sur l'ensemble du lot, 79,6\% du poids total des fragments ont pu être identifiés. La représentation des membres, 65,2\% du poids des fragments reconnus, est presque conforme au poids théorique $(62,6 \%)$. Avec $33,3 \%$ du poids des fragments identifiés, celle du crâne est anormalement forte (poids théorique : 20,4\%). Le tronc est, pour sa part extrêmement sous-représenté : $1,5 \%$ au lieu des $17 \%$ attendus. Le poids d'os représente $21,7 \%$ du poids moyen d'un squelette d'adulte brûlé (1627,1 g selon McKinley 1993), et comme la structure de recouvrement n'a livré que très peu d'os
(7,1 g qui se répartissent entre les défunts des tombes 22 et 23), on a, là, la quasi-totalité des restes de ce défunt déposés dans la sépulture (fig. 36, C).

Le poids moyen des fragments déterminés, toutes régions anatomiques confondues, est de $0,26 \mathrm{~g}(0,26 \mathrm{~g}$ pour la tête, $0,10 \mathrm{~g}$ pour le tronc et $0,27 \mathrm{~g}$ pour les membres).

\subsection{Le loculus}

Le loculus (US 17140) est une cuvette de plan ovalaire, mesurant $1,4 \mathrm{~m}$ sur son grand axe ouest-est, et $0,8 \mathrm{~m}$ sur le petit axe nord-sud, peu profonde, $8 \mathrm{~cm}$ au maximum. Le fond est à peu près plat et les parois d'inclinaison très variable, par endroits verticales (fig. 33, 34 et 36, A). Son remplissage comprend deux couches.

À la base (US 17145) prend place une terre limoneuse alluviale de couleur gris moyen, mêlée de cendres et de charbons de bois millimétriques dispersés. Cette couche renferme de nombreux fragments osseux humains incinérés, parfois de dimensions importantes, épars sur l'ensemble de la surface, et quelques petits tessons de vases non tournés.

Sur le sommet de cette strate reposent deux concentrations de pierres calcaires issues de la colline du Dévès, l'une à l'ouest, l'autre au centre du loculus, chacune constituée d'un seul lit d'éléments (US 17144) (fig. 36, A). Ces pierres portent des traces de feu et certaines ont éclaté à la chaleur mais les morceaux sont restés jointifs en place. Une terre semblable à celle de l'US 17145 lie entre eux ces éléments. La concentration centrale, mais aussi le dessus de l'US 17145 plus à l'est, sont partiellement recouverts par de gros morceaux de charbons de bois décimétriques provenant de morceaux de bûches en place. Sous ces bûches, le contact avec la couche US 17145 et avec la terre liant les pierres de l'US 17144 est fortement rougi. Ce sont des morceaux de bûches incandescents qui ont été placés dans le comblement de la fosse, ce qui a occasionné la fragmentation des pierres, dont les morceaux se sont maintenus en place, et la rubéfaction de l'interface. Le sédiment de l'US 17144 enrobe de nombreux morceaux d'os humains incinérés et quelques tessons non tournés de petite taille. Une fusaïole se trouve entre les deux concentrations pierreuses (fig. $\mathbf{3 4}$ et $\mathbf{3 6}, \mathrm{n}^{\circ} 5$ ). Sur ou entre les pierres de la concentration occidentale, prennent place quatre morceaux de bracelets en bronze se rapportant à deux ou trois exemplaires (fig. $\mathbf{3 4}$ et $\mathbf{3 6}, \mathrm{n}^{\circ} 1$ à 3 ). L'un de ces morceaux a subi un début de fusion. Sur le dessus de la concentration centrale de pierres, parmi les bûches 


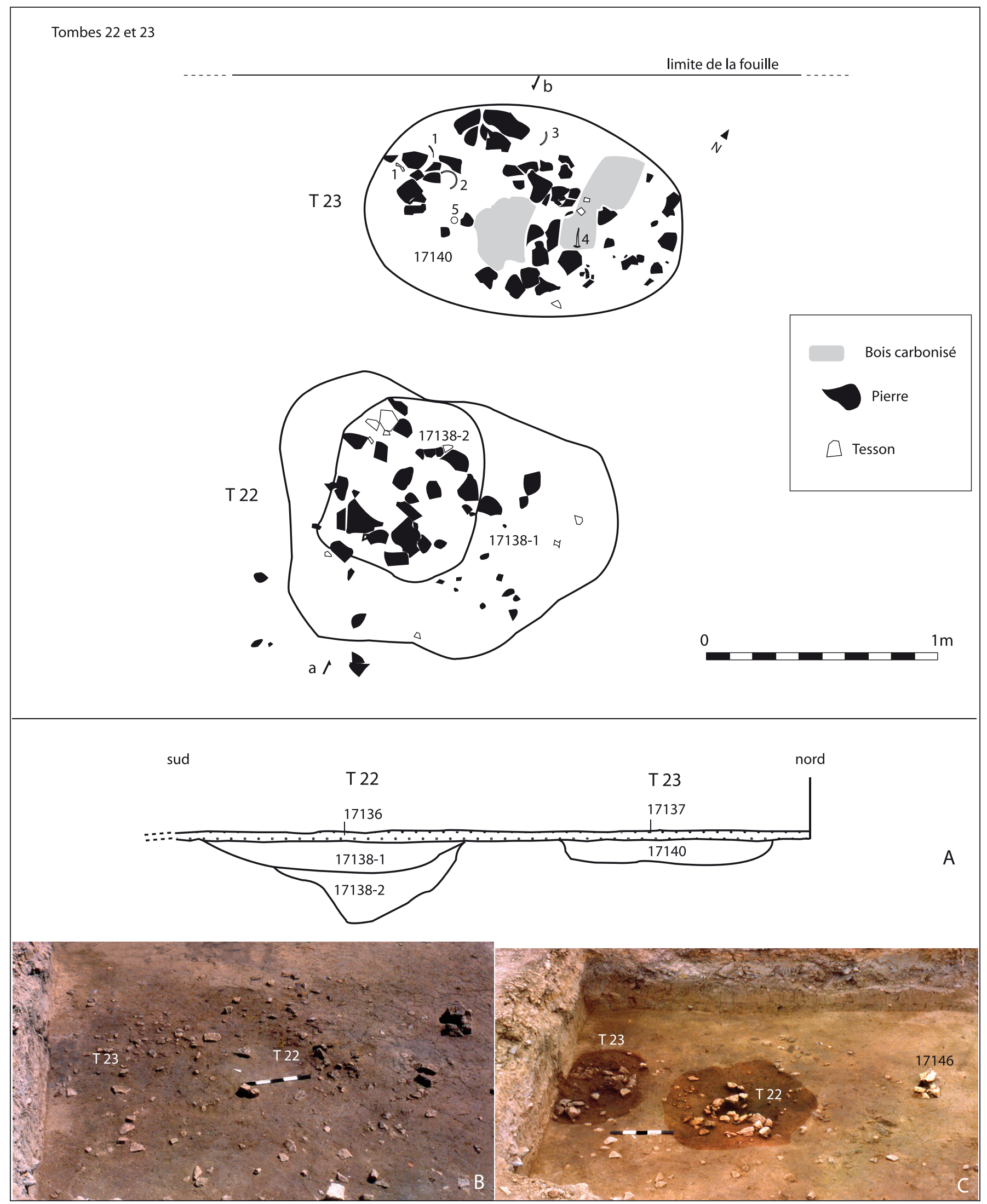

Fig. 34. Tombes 22 et 23. A : plan et coupes. B : structure de recouvrement en cours de fouille, vue du sud-ouest. $\mathrm{C}$ : loculus des tombes 22 et 23 en cours de fouille, vus sud-ouest). 


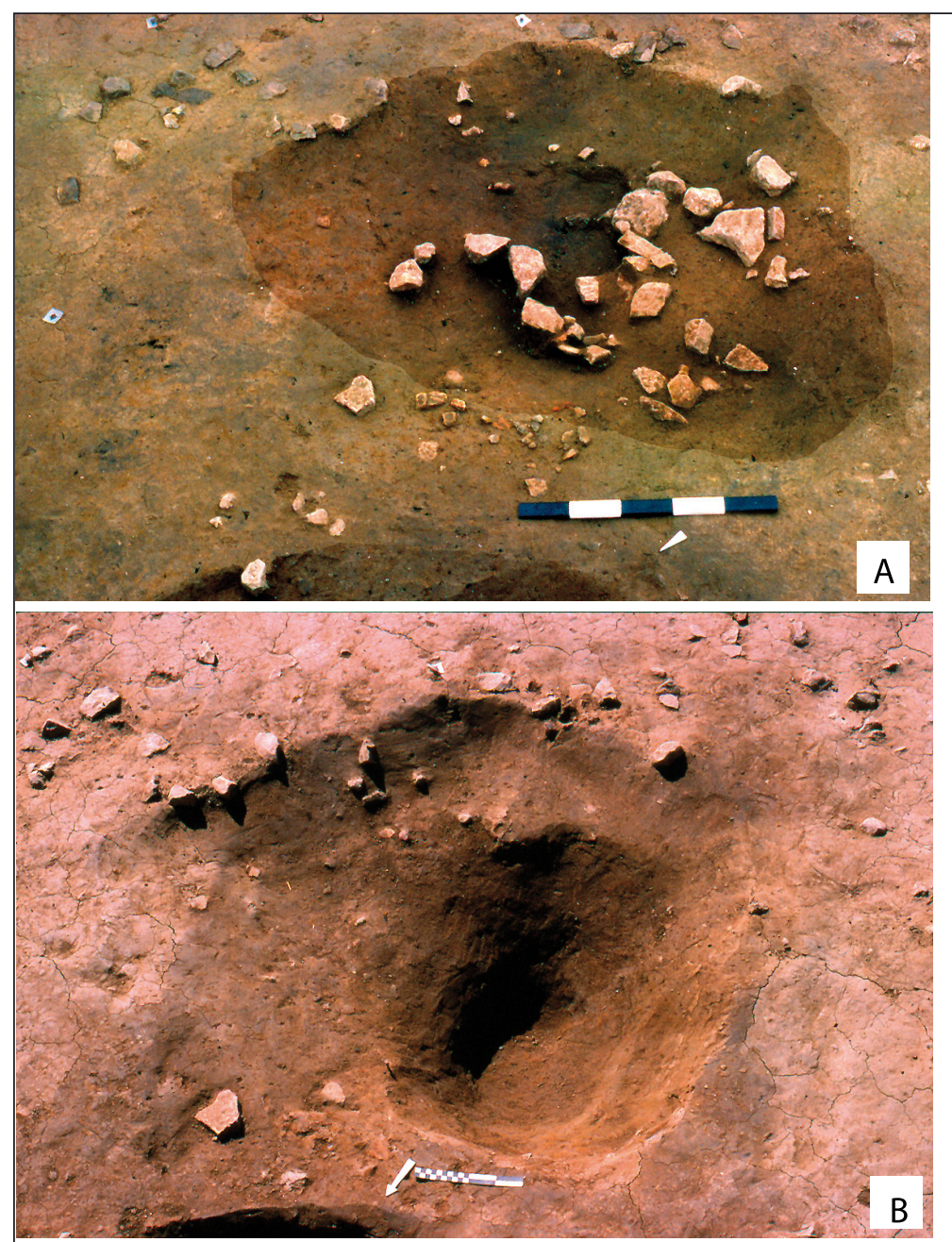

T 22

\begin{tabular}{|c|c|c|c|}
\hline T 22 & \multicolumn{3}{|c|}{ Loculus 17138 } \\
\hline Catégorie & P oids & $\% /$ id. & $\%$ /total \\
\hline Crâne & 44,9 & 60,2 & 51,4 \\
\hline Mandibule & & & \\
\hline Dents & 3,2 & 4,3 & 3,7 \\
\hline Total tête & 48,1 & 64,5 & 55 \\
\hline Vertèbres & 1 & 1,3 & 1,1 \\
\hline Côtes & 3,2 & 4,3 & 3,7 \\
\hline Sternum & & & \\
\hline Total tronc & 4,2 & 5,6 & 4,8 \\
\hline Scapula & & & \\
\hline Clavicule & & & \\
\hline Humérus & & & \\
\hline Radius & & & \\
\hline Ulna & & & \\
\hline Carpe & & & \\
\hline Métacarpe & & & \\
\hline Phal. main & 0,4 & 0,5 & 0,5 \\
\hline Tot. memb. sup. & 0,4 & 0,5 & 0,5 \\
\hline Coxal & & & \\
\hline Fémur & & & \\
\hline Patella & & & \\
\hline Tibia & & & \\
\hline Fibula & & & \\
\hline Tarse & & & \\
\hline Métatarse & & & \\
\hline Phal. pied & & & \\
\hline Tot. memb. inf. & & & \\
\hline MTC MTT P hal. & & & \\
\hline membre indét. & 21,9 & 29,4 & 25,1 \\
\hline Total membres & 22,3 & 29,9 & 25,5 \\
\hline & & & \\
\hline Total déterminé & 74,6 & 100 & 85,3 \\
\hline indét. et es quilles & 12,8 & & 14,6 \\
\hline Total indét. & 12,8 & & 14,6 \\
\hline & & & \\
\hline TOTAL & 87,4 & & 100 \\
\hline & & & C \\
\hline
\end{tabular}

T 22 Loculus
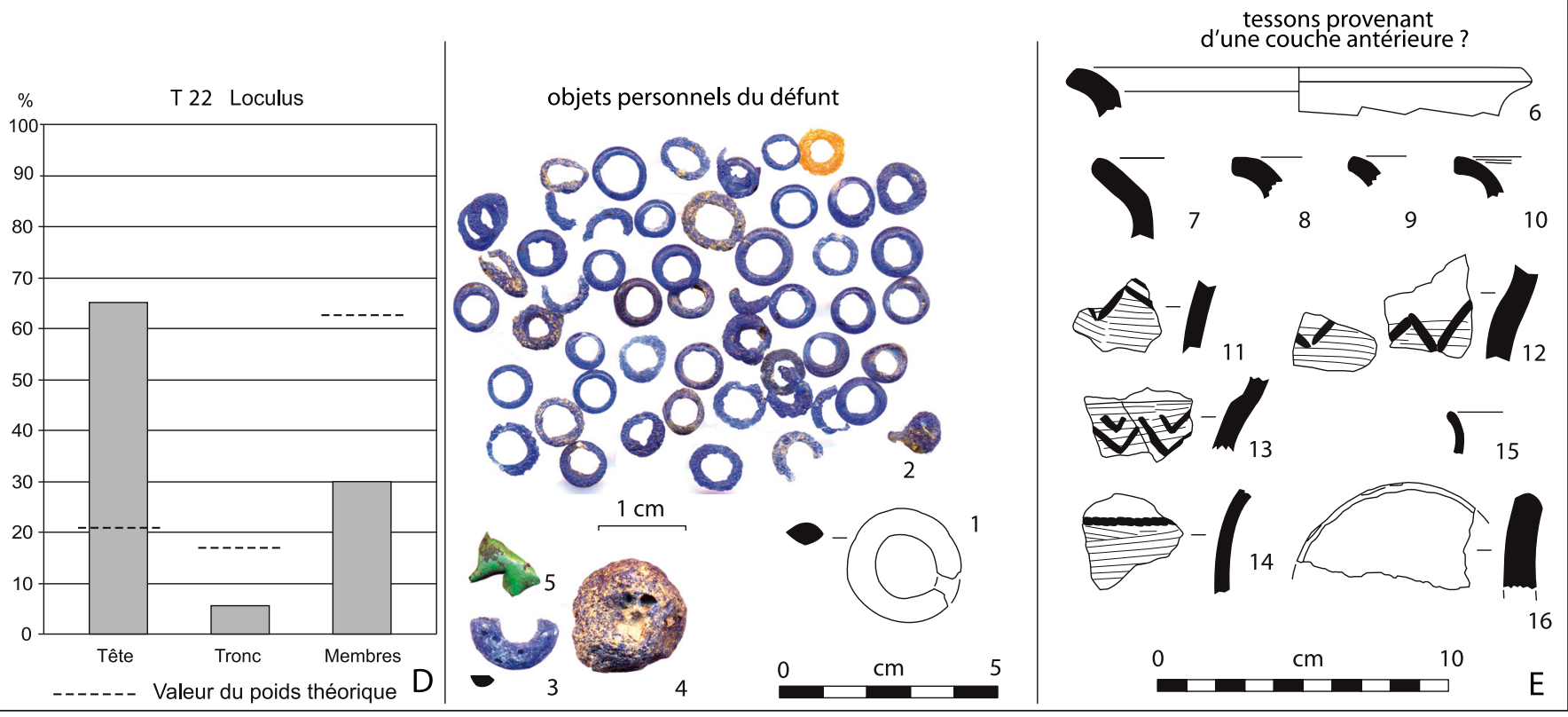

Fig. 35. Tombe T22. A : sommet du remplissage du loculus, vue prise du nord-ouest. B : loculus en fin de fouille, vue prise du nord-ouest. C : tableau de détermination des os humains (poids en grammes ; calcul des \% sur la masse). D : répartition du poids des os par grandes régions anatomiques (\% par rapport au total déterminé). $E$ : mobilier (1 et $5:$ bronze ; 2-4 : verre ; 6-14 et 16 : céramique non tournée ; 15 : céramique à pâte claire massaliète). 
carbonisées, figure aussi un gros clou en fer (fig. 34 et $\mathbf{3 6}, \mathrm{n}^{\circ} 4$ ). Vers la périphérie sud et est du loculus, le sédiment est moins sombre, et les charbons et les os incinérés sont plus rares.

\section{Mobilier du loculus}

Objets personnels, parure :

- 2 morceaux jointifs d'un bracelet en bronze (fig. 36, $\mathrm{n}^{\circ} 1$ ), incomplet; section lenticulaire; pas de décor; diam. int. : $70 \mathrm{~mm}$; haut. de la tige : $6 \mathrm{~mm}$. Un des deux morceaux est en fusion, tandis que l'autre ne porte pas trace d'un feu secondaire : le bracelet a donc été cassé avant d'être placé sur le bûcher ;

- Moitié d'un second bracelet en bronze (fig. 36, $\mathrm{n}^{\circ} 2$ ), incomplet; section lenticulaire; pas de décor; diam. int. : $63 \mathrm{~mm}$; haut. de la tige : 6 à $7,5 \mathrm{~mm}$;

- Segment de bracelet en bronze (fig. 36, $\mathrm{n}^{\circ} 3$ ) ; section lenticulaire ; pas de décor ; diam. int. : env. $70 \mathrm{~mm}$; haut. de la tige : $7 \mathrm{~mm}$. Ce morceau peut appartenir au bracelet précédent ou à un troisième exemplaire.

Objet personnel, ustensile :

- 1 grosse fusaïole en terre cuite (fig. 36, $\mathrm{n}^{\circ} 5$ ) ; biconique à carène très adoucie ; diam. : $37 \mathrm{~mm}$; haut. : $24 \mathrm{~mm}$.

Objet métallique d'usage non déterminé :

- 1 grand clou en fer (fig. $\left.36, n^{\circ} 4\right)$; tête discoïde, légèrement bombée; tige de section quadrangulaire; diam. tête : $21 \mathrm{~mm}$; long. : $69 \mathrm{~mm}$.

Matériel céramique : tessons de vases isolés :

- 1 tesson d'urne non tournée de forme probable CNT-LOR U5d : bord déversé à lèvre arrondie ; diam. ouv. : $150 \mathrm{~mm}$ (fig. $\mathbf{3 6}, \mathrm{n}^{\circ}$ 6) ;

- 3 tessons de col et bord déversé d'urne non tournée de forme probable CNT-LOR U5d : col tronconique rentrant à surface lissée, bord déversé à lèvre aplatie ; diam. ouv. : $160 \mathrm{~mm}$ (fig. 36, $\mathrm{n}^{\circ} 7$ ) ;

- 1 tesson de bord couvercle non tourné de forme probable CNT-LOR V2b, bord dans la continuité d'une paroi, semble-t-il, concave, lèvre aplatie ; diam. ouv. : env. $180 \mathrm{~mm}$ (fig. 36, $\left.\mathrm{n}^{\circ} 8\right)$;

- 1 tesson de grande coupe non tournée de forme CNT-LOR C1 ; panse arrondie convexe, embouchure évasée avec bord à lèvre aplatie et élargie; diam. ouv. : 250 à $300 \mathrm{~mm}$ (fig. 36, n ${ }^{\circ}$ 9) ;

-25 petits tessons et quelques esquilles de panses se rapportant, semble-t-il, à autant de vases non tournés, urnes et coupes (non dessinés) ;

-1 petit fragment d'amphore massaliète (non dessiné).
Os d'animaux (détermination A. Gardeisen) :

- 11 fragments d'un crâne de porc juvénile, carbonisé à blanc (fragments crâniens et des mandibules, bourgeons dentaires et 58 esquilles);

-1 troisième prémolaire inférieure de chien, non brûlée ;

- Indéterminés : 10 esquilles brûlées, couleur noire, qui se différencient nettement du lot des esquilles attribuées à la fragmentation du crâne de porcelet.

\section{La structure de recouvrement des tombes T22 et T23}

\subsection{La structure}

Une même structure de surface recouvre les loculus des tombes T22 et T23, et déborde largement tout autour sur le sol de la nécropole, formant une aire probablement ovalaire, de $3 \mathrm{~m}$ sur l'axe ouest-est, et de plus de $3 \mathrm{~m}$ sur l'axe nord-sud, l'extension vers le nord ne pouvant être appréciée du fait de la limite de la fouille (fig. $\mathbf{3 3}$ et $\mathbf{3 7}$, B). Elle est matérialisée par différents éléments qui en marquent la base :

- de nombreuses pierres centimétriques, jusqu'à $15 \mathrm{~cm}$ d'arête, reposant sur le dessus du comblement des deux loculus et sur le sol environnant, sur l'ensemble de cette aire ;

- une urne non tournée incomplète, écrasée (fig. 37, $\left.\mathrm{A}, \mathrm{n}^{\circ} 1\right)$, dont l'aire de répartition des morceaux recouvre partiellement les deux loculus 22 et 23 , et le sol de la nécropole entre ces deux structures (fig. 33, US 17137);

- sur le sol de la nécropole, entre les deux loculus, 29 petits morceaux et esquilles d'os humains incinérés ;

- trois perles (fig. 37, A, n 3 à 5), et un tesson de vase non tourné (fig. 37, A, n ${ }^{\circ} 6$ ), sur le dessus du comblement du loculus de la tombe 22 ;

- à proximité immédiate de ce loculus, sur le sol de la nécropole, deux autres petits objets d'habillement ou de parure, une perle (fig. 37, A, ${ }^{\circ} 7$ ) et un élément de ceinture (fig. 37, A, n ${ }^{\circ}$ ).

Par ailleurs, la terre alluviale qui surmonte le loculus de la tombe T22 et le sol environnant sur deux ou trois centimètres d'épaisseur se différencie du limon recouvrant le sol de la nécropole environnant; en effet, elle est mouchetée de petits fragments de charbons de bois. 


\subsection{Restes osseux humains}

Les fragments osseux incinérés, pour un poids de $7,1 \mathrm{~g}$, se rapportent à un enfant et à un sujet de taille adulte; ils sont donc compatibles avec les défunts des loculus des tombes 22 et 23 . Ce sont, notamment, 8 petits morceaux crâniens, dont un de taille adulte et cinq d'enfant et 7 fragments de membres dont trois de taille adulte, et en particulier un métatarsien, et deux d'enfant.

\subsection{Mobilier}

\section{Objets personnels, parure et habillement}

- Morceau de maillon double en bronze, appartenant à une chaîne-ceinture "féminine » de type méridional ; les deux anneaux sont reliés par une moulure simple; long. cons. $12 \mathrm{~mm}$ (fig. 37, A, $\mathrm{n}^{\circ} 2$ );

- Deux petites perles en pâte de verre bleue (fig. 37, $\mathrm{A}, \mathrm{n}^{\circ} 3$ et 4$)$; section arrondie, fine et irrégulière ; diam. ext. : 5,5 mm (fig. 37, A, $\mathrm{n}^{\circ}$ 3) et 4,5 mm (fig. 37, A, $\mathrm{n}^{\circ} 4$ ). Ces perles sont semblables à celles découvertes dans le loculus de la tombe 22 ;

- Grosse perle annulaire double, en pâte de verre bleu, ornée d'un filet ondulant jaune opaque, déformée par le feu; deux sections en D; dim. actuelle de l'objet déformé : 20 et $14 \mathrm{~mm}$ d'axes ; 16 à $17 \mathrm{~mm}$ de haut (fig. 37, $\mathrm{A}, \mathrm{n}^{\circ} 5$ ) ;

- Morceau de perle en pâte de verre bleu opaque, déformée par le feu ; diam. : $10 \mathrm{~mm}$ (fig. 37, A, $\mathrm{n}^{\circ}$ 7).

\section{Matériel céramique}

Portion de vase :

- Portion d'une urne non tournée de forme CNT-LOR U5d, variante $d, 107$ tessons, souvent de très petite taille, représentant environ $50 \%$ du récipient : col cylindrique avec, à sa partie supérieure, cassure ancienne horizontale retaillée pour former une lèvre oblique, panse ovoïde à peignage horizontal, épaule décorée d'impressions au peigne disposées selon une ligne brisée; sur la partie conservée du fond, trait incisé large et peu profond, probable croix ; diam. max. rest. : $180 \mathrm{~mm}$; haut. rest. : $230 \mathrm{~mm}$ (fig. 37, A, $\mathrm{n}^{\circ} 1$ ).

Tessons de vases isolés :

-2 tessons de vases à vernis noir de l'atelier de Rosas, de forme non déterminable, dont un morceau de fond porteur d'une estampille très endommagée, sans doute palmette, à la lecture très hypothétique (fig. $37, \mathrm{n}^{\circ} 8$ ) ;

- 1 tesson d'un grand vase non tourné fermé ; bord rentrant ; diam. max. est. : $360 \mathrm{~mm}$ (fig. 37, $\mathrm{A}, \mathrm{n}^{\circ}$ 6) ;
-4 petits tessons informes de quatre vases non tournés (non dessinés).

\subsection{Le dispositif annexe (US 17146)}

À proximité immédiate de la structure de recouvrement, à une douzaine de $\mathrm{cm}$ seulement de sa bordure sud-orientale, est à moitié enterré un dispositif de pierres calcaires locales, brutes et non brûlées (US 17146). Il se compose d'une fosse profonde, dont les limites, non perceptibles, ne peuvent être déduites que par les pierres verticales qu'elle renferme, et d'un amoncellement de pierres à la surface du sol de la nécropole (US 17135) (fig. 37, B à E).

Un bloc de forme trapézoïdale (haut : $32 \mathrm{~cm}$; large 12 à $20 \mathrm{~cm}$; épais : $6 \mathrm{~cm}$ ) est enfoui verticalement dans le sédiment sous-jacent $\left(\mathrm{n}^{\circ} 1\right)$. Sa base se trouve à $40 \mathrm{~cm}$ sous le sol de la nécropole et son sommet à $8 \mathrm{~cm}$. Cette pierre est directement surmontée par un second bloc, de forme triangulaire (haut: $30 \mathrm{~cm}$; large : $25 \mathrm{~cm}$ au sommet ; épais : 7 à $10 \mathrm{~cm})\left(\mathrm{n}^{\circ} 2\right)$. Ce dernier, enfoncé de $7 \mathrm{~cm}$ dans le sol, émerge de $23 \mathrm{~cm}$ au-dessus de lui, selon une orientation est-ouest. Contre lui, du côté nord, est adossé un petit amas de quatre pierres calcaires non brûlées décimétriques ( $\mathrm{a}, \mathrm{b}, \mathrm{c}$ et d), posées sur le sol de la nécropole (US 17135).

Le sédiment entourant la base du bloc 2 et le bloc 1 ne se différencie pas de celui des environs, et il est stérile en documents archéologiques. La fouille en profondeur autour de ces blocs n'a pas permis de repérer les limites de la fosse dans laquelle ces éléments ont dû être placés. La présence des pierres a, b, c et d regroupées autour du bloc 2 et reposant sur la surface 17135 semblent assurer un lien chronologique de contemporanéité entre le sol de la nécropole et ce dispositif semi-enterré, dont la signification reste cependant énigmatique.

\section{La tombe T24}

\section{1. État et composantes de la tombe}

La tombe T24 abrite un seul défunt incinéré, un grand enfant entre 12 et 15 ans, de sexe non déterminable, mais la présence d'une perle dans le mobilier (voir infra) pourrait indiquer un sujet féminin. Elle comprend un loculus (US 17173) et une structure de 


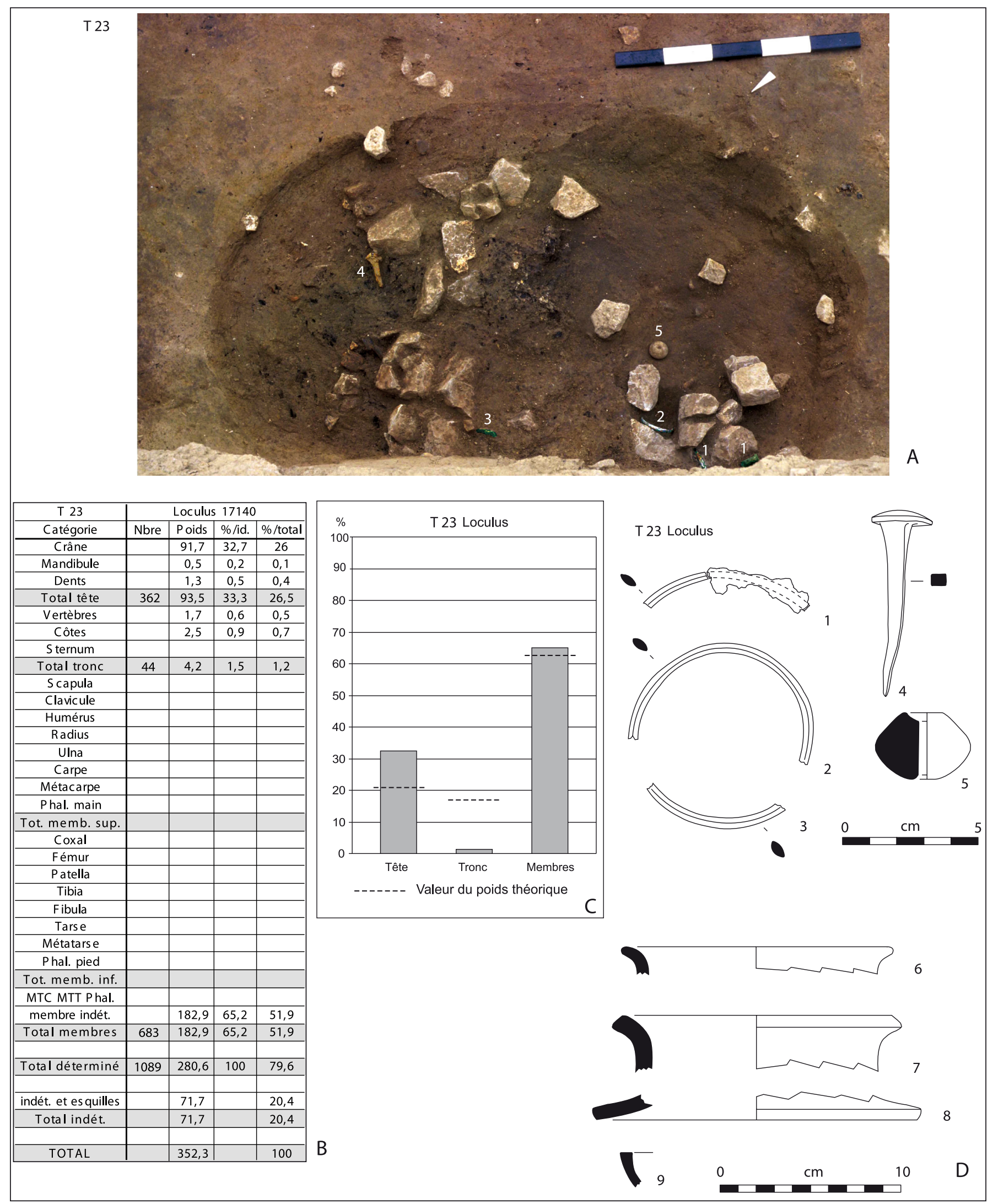

Fig. 36. Tombe T23. A : remplissage du loculus, vue prise du nord-ouest (1-3 : bracelets ; 4 : clou ; 5 fusaïole) ;

B : tableau de détermination des os humains (poids en grammes ; calcul des \% sur la masse). C : répartition du poids des os par grandes régions anatomiques (\% par rapport au total déterminé). $D$ : mobilier (1-3: bronze ; $4:$ fer ; 5 : terre cuite modelée ; 6-9 céramique non tournée). 


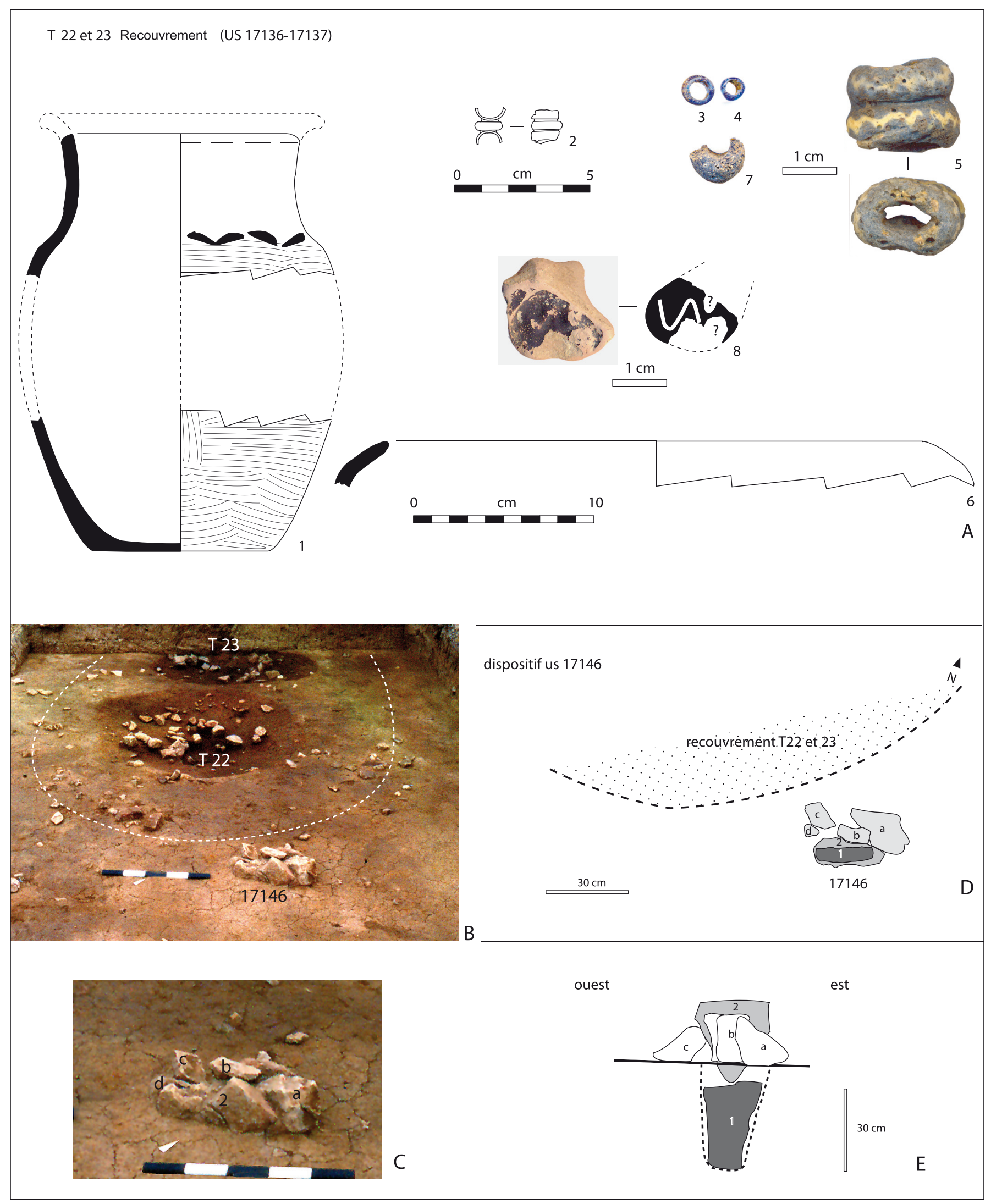

Fig. 37. Recouvrement des tombes T22 et T23. A : mobilier ( 1 et $6:$ céramique non tournée ; $2:$ bronze ; $3-5$ et $7:$ verre ; $8:$ céramique à vernis noir). $B$ : vue des tombes T22 et T23 et du dispositif annexe 17146, prise du sud-est (en tiretés blancs, la limte approximative de la structure de recouvrement. C : vue du dispositif annexe 17146, prise du sud-est. D et $\mathrm{E}$ : plan et coupe du dispositif annexe 17146. 
recouvrement (US 17166) s'étendant largement tout autour, mais seule la moitié orientale de cette dernière a pu être entièrement fouillée, la présence de murs du bâtiment gallo-romain donnant, vers l'est, une limite aux recherches (fig. 38).

\subsection{Le défunt}

Le défunt est représenté par 327,1 g d'os brûlés (fig. 39, A). L'estimation de l'âge au décès, vers 12-15 ans, repose sur plusieurs indices, outre le caractère gracile des pièces osseuses: plusieurs fragments de diaphyses montrent une surface métaphysaire; un fragment d'épiphyse distale d'ulna et trois extrémités proximales de phalanges ne sont pas soudées; si les racines des incisives et des premières molaires sont complètement formées, avec des apex fermés, il n'en va pas de même des premières prémolaires et des deuxièmes molaires dont les racines ne sont formées qu'à $80 \%$. Le sexe anthropologique ne peut pas être précisé.

La couleur blanc crayeux révèle une température de crémation supérieure à $650^{\circ}$ selon le barème de E. Bonnucci et G. Grazziani (1975), et les morceaux de diaphyses sont très souvent fissurés en ondes concentriques, indice d'une incinération d'os frais.

Le loculus a livré 319,6 g d'os incinérés, et la partie fouillée de la structure de recouvrement seulement $7,5 \mathrm{~g}$. On ne peut affirmer toutefois que la quasi totalité des restes osseux ait été déposée dans le loculus, car on ignore si la moitié non fouillée du recouvrement est à l'image de la partie occidentale.

Le poids d'os déposés dans le loculus représente 19,6\% du poids moyen d'un squelette d'adulte brûlé, $1627,1 \mathrm{~g}$ selon McKinley (1993). Les taux d'identification sont de $91,4 \%$ du poids total des fragments pour le loculus. Le crâne, surtout, mais aussi dans une moindre mesure, les membres sont sur-représentés, avec, respectivement, 27,6 et $66,4 \%$ du poids identifié (pour des poids théoriques de 20,4 et $62,6 \%$ ), tandis que la représentation du tronc est très faible, $6 \%$ (poids théorique : $17 \%$ ) (fig. 39, B).

Les os ont été placés dans le loculus hors de tout contenant, mais le mode de dépôt traduit deux gestes successifs. Dans la partie inférieure du loculus (US 17174), un magma très dense de cendres gris blanchâtre incluant des charbons de bois et de très nombreux morceaux d'os, souvent amalgamés entre eux, de grosses dimensions, traduit un prélèvement en bloc, sur le bûcher, de matières brûlantes (fig. 39, A). Dans la partie supérieure (US 17173), les restes osseux, de taille plus réduite, sont éparpillés dans un sédiment limoneux versé au-dessus de ce magma, qui s'est coloré en rouge lie de vin, sans doute par suite de la chaleur. Les deux lots diffèrent en volume, 90,4 g pour la partie supérieure et 229,2 g pour l'amas de la partie inférieure, mais aucune incompatibilité n'existe entre eux, et, par ailleurs, les restes les composant proviennent des mêmes régions du corps.

Toutes régions anatomiques confondues, le poids moyen des fragments déterminés provenant du loculus est semblable à celui des os du dispositif de recouvrement et s'élève à $0,17 \mathrm{~g}$ pour le premier et $0,18 \mathrm{~g}$ pour le second (provenant du loculus : $0,18 \mathrm{~g}$ pour la tête, $0,11 \mathrm{~g}$ pour le tronc et $0,18 \mathrm{~g}$ pour les membres ; provenant du recouvrement : $0,15 \mathrm{~g}$ pour la tête, $0,10 \mathrm{~g}$ pour le tronc et $0,20 \mathrm{~g}$ pour les membres). Le poids moyen des 8 fragments identifiés comme provenant des membres inférieurs est de $1,38 \mathrm{~g}$.

\subsection{Le loculus}

Le loculus (US 17173-17174) est une cuvette conique, de $70 \mathrm{~cm}$ de diamètre à l'ouverture, profonde de $30 \mathrm{~cm}$ au maximum, aux parois inclinées à $45^{\circ}$ (fig. 38 et $\mathbf{4 0}, \mathrm{A}$ ).

Son comblement comprend deux parties. La partie inférieure (US 17174), épaisse de $20 \mathrm{~cm}$ au maximum, est composée d'un magma cendreux riche en os humains incinérés et en charbons de bois. Au milieu de ce comblement, prennent place cinq pierres calcaires provenant de la colline du Dévès et dépourvues de traces d'éclatement au feu. Ces éléments mesurent jusqu'à $10 \mathrm{~cm}$ d'arête. Les pierres situées sur les bords de la fosse épousent le pendage très oblique de ceux-ci ; les autres sont horizontales. Sous ces pierres, vers le centre, gisent, sans ordre et selon des pendages variés, cinq morceaux d'une urne non tournée incomplète (fig. 40, B, n ${ }^{\circ} 1,1$ b et 1c), certains jointifs. Le fond du comblement se compose du même magma cendreux et osseux, mais il est dépourvu de pierres. Une couche de terre limoneuse de couleur rouge lie de vin forme la partie supérieure du remplissage (US 17173). Elle est mouchetée de petits charbons de bois millimétriques ou plus rarement centimétriques, épars, et inclut des morceaux d'os humains incinérés de petite taille, relativement abondants. Elle enrobe cinq pierres décimétriques calcaires provenant de la colline du Dévès, qui ne portent pas de trace de rubéfaction, et contient deux tessons de deux urnes non tournées (fig. 40, B, $\mathrm{n}^{\circ}$ 2). 


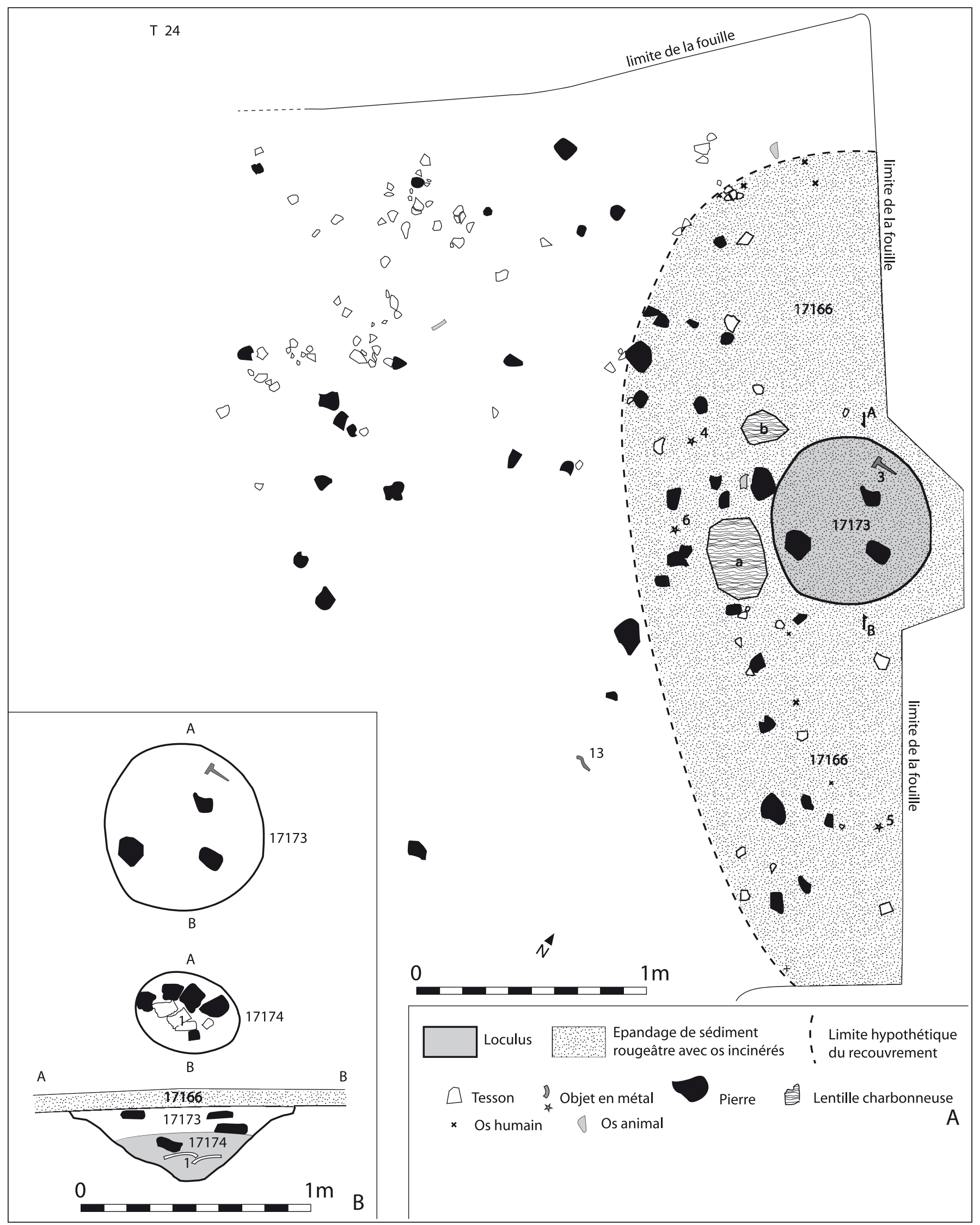

Fig. 38. Tombes T24. A : plan de la structure de recouvrement et du loculus. B : plan du loculus à deux niveaux du remplissage, et coupe. 


\begin{tabular}{|c|c|c|c|c|c|c|c|c|c|c|c|c|c|c|c|c|c|}
\hline T 24 & \multicolumn{3}{|c|}{ Loculus 17173} & \multicolumn{3}{|c|}{ Loculus 17174} & \multicolumn{4}{|c|}{ Total loculus } & \multicolumn{4}{|c|}{ Recouvrement } & \multicolumn{3}{|c|}{ Total tombe } \\
\hline Catégorie & Poids & $\%$ /id. & $\% /$ total & \begin{tabular}{|l|l|} 
Poids \\
\end{tabular} & $\% /$ id. & $\% /$ total & Nbre & Poids & $\% /$ id. & $\% /$ total & Nbre & $\begin{array}{ll}\text { Poids } \\
\end{array}$ & $\% /$ id. & $\% /$ total & \begin{tabular}{|l|} 
Poids \\
\end{tabular} & $\% /$ id. & $\% /$ total \\
\hline Crâne & 17,5 & 21,9 & 19,4 & 57 & 26,9 & 24,9 & 400 & 74,5 & 25,5 & 23,3 & 5 & 0,8 & 14,8 & 10,7 & 75,3 & 25,3 & 23 \\
\hline Mandibule & & & & 0,6 & 0,3 & 0,3 & 1 & 0,6 & 0,2 & 0,2 & & & & & 0,6 & 0,2 & 0,2 \\
\hline Dents & 1,8 & 2,3 & 2 & 3,7 & 1,7 & 1,6 & 49 & 5,5 & 1,9 & 1,7 & 1 & 0,1 & 1,6 & 1,3 & 5,6 & 1,9 & 1,7 \\
\hline Total tête & 19,3 & 24,2 & 21,3 & 61,3 & 28,9 & 26,8 & 450 & 80,6 & 27,6 & 25,2 & 6 & 0,9 & 16,7 & 12 & 81,5 & 27,4 & 24,9 \\
\hline Vertèbres & 2,9 & 3,6 & 3,2 & 0,9 & 0,4 & 0,4 & & 3,8 & 1,3 & 1,2 & & 0,1 & 1,6 & 1,3 & 3,9 & 1,3 & 1,2 \\
\hline Côtes & 2,6 & 3,3 & 2,9 & 11,1 & 5,2 & 4,8 & & 13,7 & 4,7 & 4,3 & & 0,1 & 1,6 & 1,3 & 13,8 & 4,6 & 4,2 \\
\hline \multicolumn{18}{|l|}{ S ternum } \\
\hline Total tronc & 5,5 & 6,9 & 6,1 & 12 & 5,7 & 5,2 & 161 & 17,5 & 6 & 5,5 & 2 & 0,2 & 3,2 & 2,7 & 17,7 & 6 & 5,4 \\
\hline Scapula & & & & 0,3 & 0,1 & 0,1 & 1 & 0,3 & 0,1 & 0,1 & & & & & 0,3 & 0,1 & 0,1 \\
\hline \multirow{2}{*}{\multicolumn{18}{|c|}{$\begin{array}{l}\text { Clavicule } \\
\text { Humérus }\end{array}$}} \\
\hline & & & & & & & & & & & & \multicolumn{5}{|c|}{ Humérus } & \\
\hline \multicolumn{18}{|l|}{ Radius } \\
\hline \multicolumn{18}{|l|}{ Ulna } \\
\hline \multicolumn{18}{|l|}{ Carpe } \\
\hline \multicolumn{18}{|l|}{ Métacarpe } \\
\hline \multicolumn{18}{|l|}{ Phal. main } \\
\hline Tot. memb. sup. & & & & 0,3 & 0,1 & 0,1 & 1 & 0,3 & 0,1 & 0,1 & & & & & 0,3 & 0,1 & 0,1 \\
\hline Coxal & & & & 4,7 & 2,2 & 2,1 & 3 & 4,7 & 1,6 & 1,5 & & & & & 4,7 & 1,6 & 1,4 \\
\hline Fémur & & & & 1,5 & 0,7 & 0,7 & 1 & 1,5 & 0,5 & 0,5 & & & & & 1,5 & 0,5 & 0,5 \\
\hline \multicolumn{18}{|l|}{ Patella } \\
\hline Tibia & & & & 1,8 & 0,8 & 0,8 & 2 & 1,8 & 0,6 & 0,6 & & & & & 1,8 & 0,6 & 0,6 \\
\hline \multicolumn{18}{|l|}{ Fibula } \\
\hline Tarse & & & & 3 & 1,4 & 1,3 & 2 & 3 & 1 & 0,9 & & & & & 3 & 1 & 0,9 \\
\hline \multicolumn{18}{|l|}{ Métatarse } \\
\hline \multicolumn{18}{|l|}{ Phal. pied } \\
\hline Tot. memb. inf. & & & & 11 & 5,2 & 4,8 & 8 & 11 & 3,8 & 3,4 & & & & & 11 & 3,7 & 3,4 \\
\hline MTC MTT P hal. & 0,3 & 0,4 & 0,3 & 7,7 & 3,6 & 3,4 & & 7,7 & 2,6 & 2,4 & & & & & 7,7 & 2,6 & 2,4 \\
\hline membre indét. & & & & 119,9 & 56,5 & 52,3 & & 174,7 & 59,9 & 54,7 & & 4,3 & 79,6 & 57,3 & 179 & 60,2 & 54,8 \\
\hline Total membres & 55,1 & 69 & 61 & 138,9 & 65,5 & 60,6 & 1062 & 194 & 66,4 & 60,7 & 22 & 4,3 & 79,6 & 57,3 & 198,3 & 66,7 & 60,6 \\
\hline Total déterminé & 79,9 & 100 & 88,4 & 212,2 & 100 & 92,6 & 1673 & 292,1 & 100 & 91,4 & 30 & 5,4 & 100 & 72 & 297,5 & 100 & 90,9 \\
\hline & & & & & & & & & & & & & & & & & \\
\hline indét. et es quilles & 10,5 & & & 17 & & & & 27,5 & & & & 2,1 & & & 29,6 & & 9,1 \\
\hline Total indét. & 10,5 & & 11,6 & 17 & & 7,4 & & 27,5 & & 8,6 & & 2,1 & & 28 & 29,6 & & 9,1 \\
\hline & 00 & & 100 & & & 100 & & 30 & & 100 & & 75 & & 100 & 237 & & 100 \\
\hline
\end{tabular}
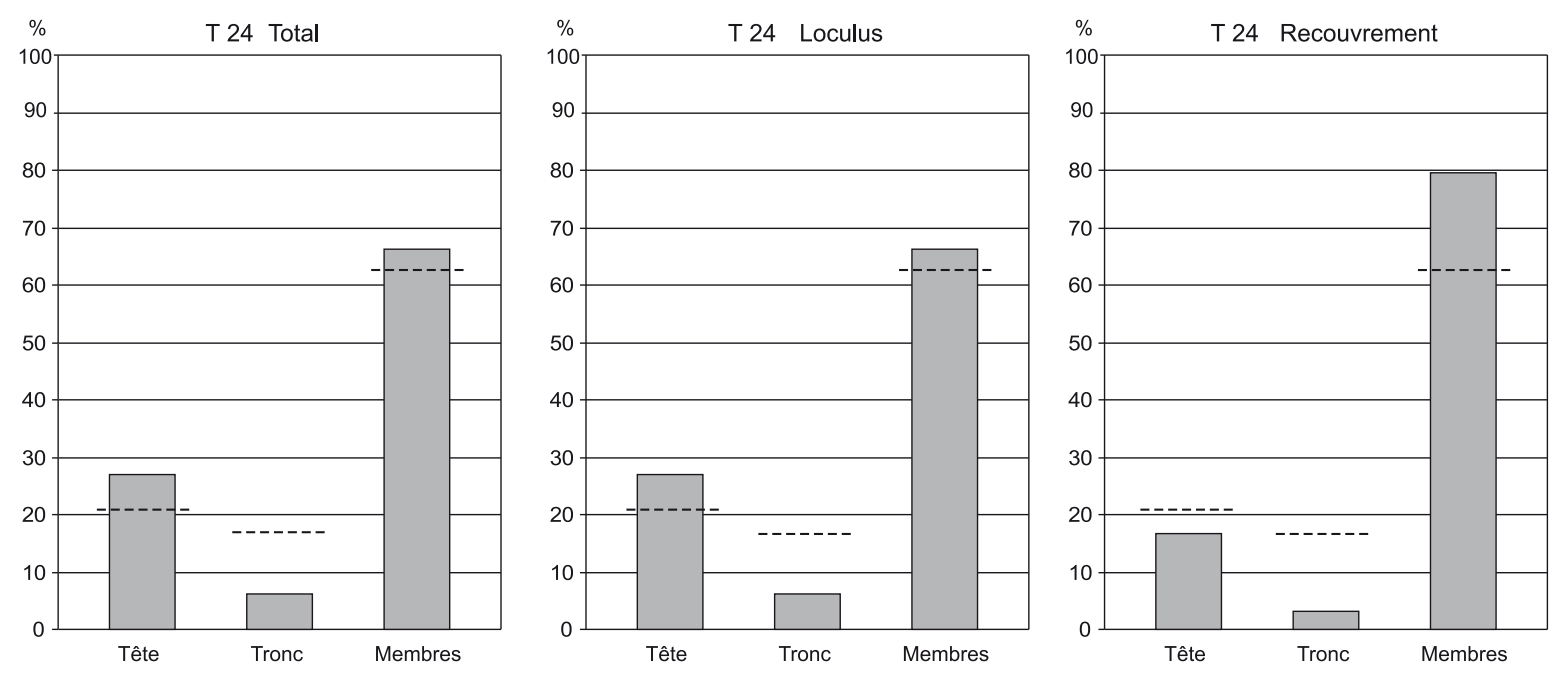

------- Valeur du poids théorique

Fig. 39. Tombe T24. A : tableau de détermination des os humains (poids en grammes; calcul des \% sur la masse). $\mathrm{B}$ : répartition du poids des os par grandes régions anatomiques (\% par rapport au total déterminé). 


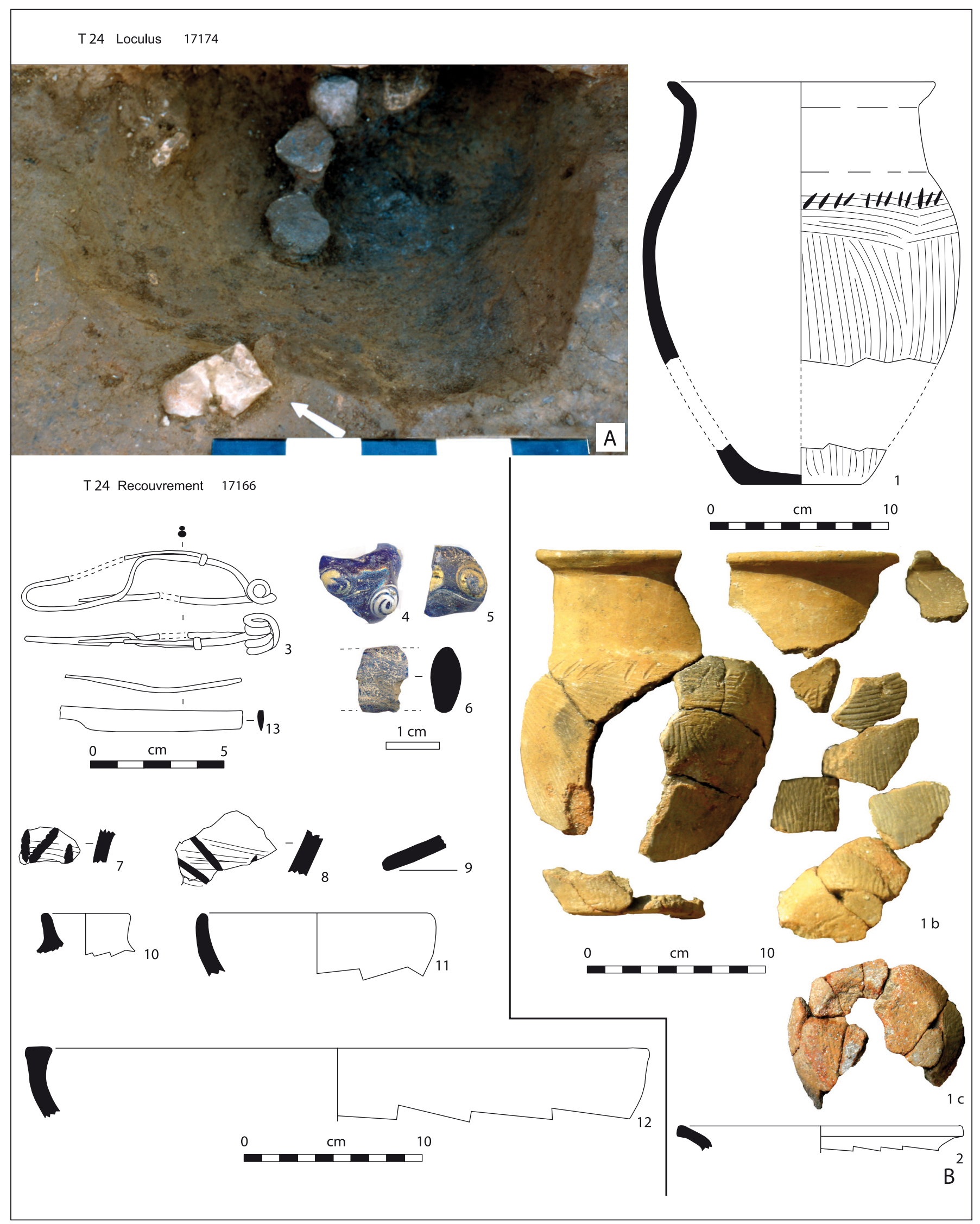

Fig. 40. Tombe T24. A : loculus en cours de fouille, vue prise du sud-ouest. B : mobilier (1, 2 et $7-12:$ céramique non tournée ; 3 et $13:$ fer ; 4-6 : verre). 


\section{Mobilier du loculus}

Matériel céramique : une portion de vase et tessons isolés de deux vases :

- Portion d'une urne non tournée de forme CNTLOR U5d, variante d, 28 tessons, la plupart jointifs, représentant environ $50 \%$ du vase : col tronconique rentrant à surface polie, bord évasé à lèvre arrondie, panse ovoïde à peignage horizontal sur l'épaule et vertical ailleurs, épaule décorée d'un rang horizontal de petites impressions fusiformes obliques, fond plat avec stries de peignage par endroit ; diam. max. : $178 \mathrm{~mm}$; haut. estimée : $225 \mathrm{~mm}$ (fig. 40, $\mathrm{B}, \mathrm{n}^{\circ} 1,1 \mathrm{~b}$ et $1 \mathrm{c}$ ). Les traces d'un coup porté sur le fond du vase depuis l'extérieur a provoqué un large éclatement circulaire et une perforation. Ce choc semble avoir causé le bris du récipient (fig. 40, $\left.\mathrm{B}, \mathrm{n}^{\circ} 1 \mathrm{c}\right)$;

- 1 tesson d'urne non tournée de forme probable CNT-LOR U5d: bord évasé à lèvre arrondie; diam. ouv. : $160 \mathrm{~mm}$ (fig. 40, $\left.\mathrm{B}, \mathrm{n}^{\circ} 2\right)$;

-1 tesson de panse de vase non tourné, à surface extérieure lissée (non dessiné).

Os d'animaux (détermination A. Gardeisen) :

- 5 fragments et 14 esquilles de neurocrâne de capriné adulte, brûlés de couleur bleue ;

- Indéterminé : 1 fragment d'os crânien non brûlé ;

- Malacofaune : 1 fragment de coquillage.

\subsection{La structure de recouvrement}

Le loculus et le paléosol environnant sont surmontés, sur un à deux centimètres d'épaisseur, par une fine couche de limon de couleur rouge lie de vin (fig. 38). Dans la partie qui a pu être fouillée, ce recouvrement s'étend sur une aire allongée nord - sud, d'environ $4 \mathrm{~m}$, débordant d'environ 1,2 $\mathrm{m}$ au nord du loculus et $1,8 \mathrm{~m}$ au sud. Sa largeur reste inconnue, du fait de la limite de la fouille vers l'est ; cependant, à l'ouest, cette superstructure ne déborde le loculus que de $0,5 \mathrm{~m}$.

Cette aire inclut des nodules d'argile plus sombres, de nombreux petits charbons de bois épars, quelques pierres plates calcaires centimétriques, de rares petits fragments d'os humains incinérés, surtout au-dessus du loculus et à ses abords immédiats, 40 tessons de vases non tournés en position horizontale, appartenant à 38 récipients, et quatre petits objets de parure ou d'habillement. Deux petits amas de charbons de bois, d'un centimètre d'épaisseur, prennent place en bordure occidentale du loculus.

\section{Mobilier de la structure de recouvrement}

Objets de parure et d'habillement :

- Au-dessus du loculus, fibule en fer (fig. 40, B, $\mathrm{n}^{\circ} 3$ et fig. 115), presque complète (il manque la partie médiane du pied); ressort bilatéral à quatre spires et corde extérieure ; arc en tige mince de section circulaire ; long pied recourbé et fixé au sommet de l'arc par deux petites griffes enveloppantes ; long. : $95 \mathrm{~mm}$; larg. au ressort : $16 \mathrm{~mm}$; la lacune au niveau du pied ne permet pas de savoir si celui-ci était orné d'un nodule ; type 13c ou 14 de R. Gebhard (1991);

- À $45 \mathrm{~cm}$ à l'ouest du loculus, un morceau d'une grosse perle cylindrique en pâte de verre bleu, à trois rangs de protubérances ornées d'un filet blanc ou jaune en spirale (type IV-1-1 de Zepezauer 1989, p. 113-114) ; diam. : $17 \mathrm{~mm}$; haut. : $16 \mathrm{~mm}$ (fig. 40, $\mathrm{B}, \mathrm{n}^{\circ} 4$ ). Cette perle a subi l'action d'un feu secondaire ;

- À $1 \mathrm{~m}$ au sud-est du loculus, un morceau d'une seconde grosse perle cylindrique en pâte de verre bleu, à protubérances ornées d'un filet jaune en spirale (type IV-1-1 de Zepezauer 1989, p. 113-114); haut. : 13 mm (fig. 40, B, $\mathrm{n}^{\circ}$ 5). Cet objet a aussi subi l'action d'un feu secondaire ;

- À $45 \mathrm{~cm}$ au sud-ouest du loculus, un petit morceau d'une troisième grosse perle en pâte de verre bleu foncé uni, cylindrique et de section ovale ; diam. : $32 \mathrm{~mm}$; haut. : $12 \mathrm{~mm}$ (fig. 40, $\mathrm{B}, \mathrm{n}^{\circ}$ 6). Cet objet a également subi l'action d'un feu secondaire.

Objet métallique indéterminé :

- Â proximité immédiate du recouvrement, au sud, un morceau de lamelle en fer légèrement recourbée ; long. cons. : $67 \mathrm{~mm}$; haut. : $8 \mathrm{~mm}$; ép. : 2 à $3 \mathrm{~mm}$ (fig. 40, B, $\mathrm{n}^{\circ} 13$ ).

Tessons de vases isolés :

- 2 tessons d'épaule de deux urnes non tournées, décorés d'un rang horizontal d'impressions disposées en ligne brisée (fig. 40, B, $\mathrm{n}^{\circ} 7$ et 8 ) ;

- 1 tesson de grand couvercle non tourné de forme CNT-LOR V2a : bord à lèvre arrondie, dans la continuité de la paroi rectiligne (fig. 40, $\mathrm{B}, \mathrm{n}^{\circ}$ 9) ;

- 1 pied annulaire de couvercle non tourné ; diam. pied : $50 \mathrm{~mm}$ (fig. 40, B, $\left.\mathrm{n}^{\circ} 10\right)$;

- 1 tesson de petite coupe non tournée de forme CNTLOR C1 : panse arrondie convexe, embouchure évasée, bord à lèvre arrondie ; diam. max. : 135 mm (fig. 40, B, $\left.\mathrm{n}^{\circ} 11\right)$;

- 3 tessons d'une grande jatte non tournée de forme CNT-LOR J1e, variante 1: panse arrondie convexe, embouchure évasée, bord à lèvre élargie avec aplatissement horizontal ; diam. ouv. : $350 \mathrm{~mm}$ (fig. 40, $\left.\mathrm{B}, \mathrm{n}^{\circ} 12\right)$; 
- 31 tessons de panses appartenant à autant de vases non tournés (non dessinés) ;

-1 tesson de panse de petit dolium (non dessiné).

Os d'animaux (détermination A. Gardeisen) :

- 4 fragments de caprinés (1 deuxième molaire supérieure gauche adulte, 1 extrémité distale de phalange I, 1 fragment de diaphyse distale de tibia, 1 fragment de diaphyse (tubérosité deltoïdienne) d'humérus) ;

-1 couronne de troisième incisive supérieure gauche de porc jeune adulte ;

-1 fragment de vertèbre d'animal de petite taille ;

- Indéterminés : 8 esquilles.

L'ensemble est très altéré, jaune, poreux, et très friable : il s'agit probablement des effets de la chaleur, sans carbonisation directe au feu.

\section{La tombe $\mathrm{T} 25$}

\section{1. État et composantes de la tombe}

La tombe T25 abrite un seul défunt incinéré, un adulte de sexe non déterminable, mais la composition du mobilier (voir infra) paraît correspondre à un homme. Elle comprend un loculus (US 17182) et une structure de recouvrement (US 17116-17118) s'étendant largement tout autour ; cette dernière n'a pu être cependant entièrement fouillée car elle est surmontée par des murs du bâtiment gallo-romain et sa partie nord et ouest nous échappe (fig. 41).

\subsection{Le défunt}

Le défunt est représenté par 786,2 g d'os brûlés (fig. 42, A). La morphologie des os, la fermeture de l'apex des quatre racines de dents conservées, le listel soudé sur les corps vertébraux montrent qu'il s'agit d'un adulte. Le sexe anthropologique ne peut pas être précisé.

Les restes osseux ont une couleur blanc crayeux qui révèle une température de crémation supérieure à $650^{\circ}$ selon le barème de E. Bonnucci et G. Grazziani (1975) et les morceaux de diaphyses sont très souvent fissurés en ondes concentriques, indice d'une incinération d'os frais.

Le loculus a livré $765,9 \mathrm{~g}$ d'os et la partie fouillée du recouvrement 20,3 g et les deux lots sont tout à fait compatibles. Dans les deux cas, les restes osseux sont dispersés hors de tout contenant, et aucune disposition particulière des fragments n'a été perçue. Cependant, dans le loculus, ceux-ci sont souvent de grandes dimensions, à la différence de ceux du recouvrement, toujours très petits.

Le poids d'os déposés dans le loculus représente 48,3\% du poids moyen d'un squelette d'adulte brûlé, 1627,1 g selon McKinley (1993). Les taux d'identification sont de $89,6 \%$ du poids total des fragments pour le loculus et $91,6 \%$ pour la superstructure. Dans le loculus, la représentation du crâne est presque normale, $19,2 \%$ du poids identifié ; celle du tronc est très faible, $6,9 \%$ (poids théorique : $17 \%$ ) et celle des membres, en revanche, plus élevée que la valeur théorique, $73,3 \%$ au lieu des 62,6\% attendus (fig. 42, B).

Le poids moyen des fragments déterminés, toutes régions anatomiques confondues, est semblable dans le loculus et dans le dispositif de recouvrement et s'élève à $0,33 \mathrm{~g}$ pour le premier et $0,34 \mathrm{~g}$ pour le second (provenant du loculus : $0,34 \mathrm{~g}$ pour la tête, $0,19 \mathrm{~g}$ pour le tronc et $0,36 \mathrm{~g}$ pour les membres; provenant du recouvrement : $0,30 \mathrm{~g}$ pour la tête, $0,10 \mathrm{~g}$ pour le tronc et $0,35 \mathrm{~g}$ pour les membres). Pour les 31 fragments identifiés comme provenant des membres supérieurs, le poids moyen est de $0,82 \mathrm{~g}$; pour les 12 fragments identifiés comme provenant des membres inférieurs, il est de $3,09 \mathrm{~g}$.

\subsection{Le loculus}

Le loculus (US 17182) présente, à l'ouverture, un plan arrondi de 65 à $70 \mathrm{~cm}$ de diamètre. Sa profondeur maximum est de $32 \mathrm{~cm}$ (fig. 41). Le creusement comprend deux parties : la partie supérieure (US 17182-1) forme un ressaut de 5 à $10 \mathrm{~cm}$ de large, à environ $5 \mathrm{~cm}$ de profondeur ; la partie inférieure de la fosse, de 43 à $50 \mathrm{~cm}$ de diamètre à ce niveau, a des parois très abruptes et un fond arrondi. La partie supérieure est remplie d'une terre alluviale rougeâtre sombre, qui inclut des morceaux de bûches centimétriques, jusqu'à $3 \mathrm{~cm}$, étouffés par l'enfouissement, et de nombreux gros morceaux d'os humains incinérés. Dans la partie médiane du remplissage (US 17182-2), le sédiment devient gris et noir, charbonneux et cendreux, et comporte quelques petites pierres calcaires de la colline éclatées au feu. Il inclut également de très nombreux fragments osseux incinérés, certains de grandes dimensions, quelques petits tessons de céramiques non tournées ou à vernis noir, le mobilier métallique et les perles en pâte de verre. Un morceau d'épiphyse est collé à l'applique $\mathrm{n}^{\circ} 4$ par l'oxyde. Le fond du loculus (US 17182-3) est occupé par une terre cendreuse de couleur gris blanchâtre, où les fragments osseux sont moins nombreux. 


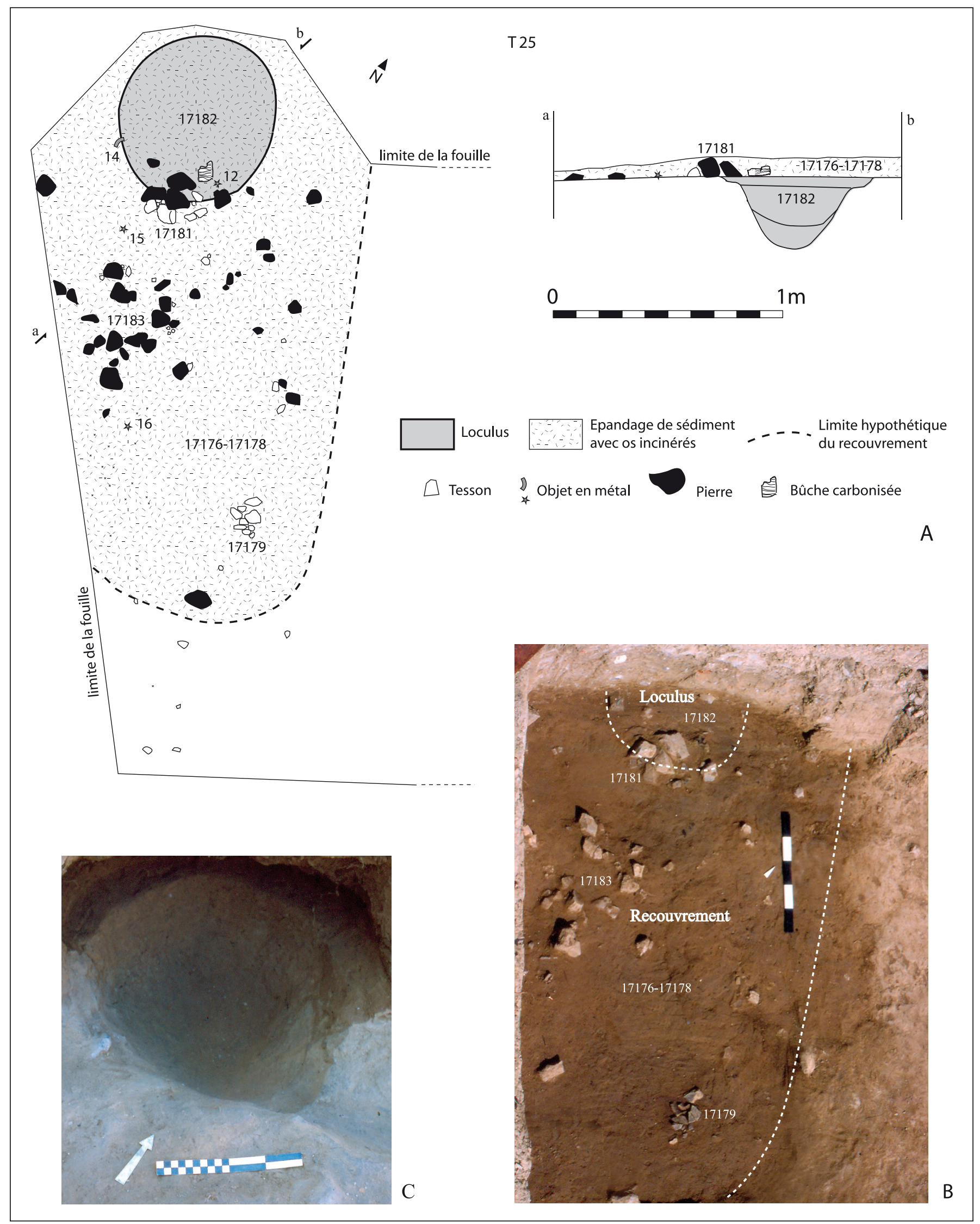

Fig. 41. Tombe T25. A : plan et coupe. B : structure de recouvrement et loculus vus du sud-est. C : loculus en fin de fouille, vu du sud-est. 
CHAPITRE 2 - TOMBES, STRUCTURES ANNEXES ET SOL DE LA NÉCROPOLE

\begin{tabular}{|c|c|c|c|c|c|c|c|c|c|c|c|}
\hline T 25 & \multicolumn{4}{|c|}{ Loculus } & \multicolumn{4}{|c|}{ Recouvrement } & \multicolumn{3}{|c|}{ Total tombe } \\
\hline Catégorie & Nbre & P oids & $\% /$ id. & $\% /$ total & Nbre & P oids & \%/id. & $\% /$ total & P oids & $\% /$ id. & $\% /$ total \\
\hline Crâne & 410 & 123,9 & 18 & 16,2 & 10 & 3,5 & 18,8 & 17,2 & 127,4 & 18,1 & 16,2 \\
\hline Mandibule & 3 & 4,6 & 0,7 & 0,6 & & & & & 4,6 & 0,7 & 0,6 \\
\hline Dents & 21 & 3,6 & 0,5 & 0,5 & 2 & 0,5 & 2,7 & 2,5 & 4,1 & 0,6 & 0,5 \\
\hline Total tête & 434 & 132,1 & 19,2 & 17,2 & 12 & 4 & 21,5 & 19,7 & 136,1 & 19,3 & 17,3 \\
\hline Vertèbres & & 28,4 & 4,1 & 3,7 & & 0,1 & & & 28,5 & 4 & 3,6 \\
\hline Côtes & & 22,4 & 3,3 & 2,9 & & & & & 22,4 & 3,2 & 2,8 \\
\hline \multicolumn{12}{|l|}{ S ternum } \\
\hline Total tronc & 274 & 50,8 & 7,4 & 6,6 & 1 & 0,1 & 0,5 & 0,5 & 50,9 & 7,2 & 6,5 \\
\hline S capula & 12 & 1,3 & 0,2 & 0,2 & & & & & 1,3 & 0,2 & 0,2 \\
\hline \multicolumn{12}{|l|}{ Clavicule } \\
\hline Humérus & 3 & 9,6 & 1,4 & 1,3 & & & & & 9,6 & 1,4 & 1,2 \\
\hline Radius & 1 & 7,5 & 1,1 & 1 & & & & & 7,5 & 1,1 & 1 \\
\hline Ulna & 1 & 1,1 & 0,2 & 0,1 & & & & & 1,1 & 0,2 & 0,1 \\
\hline Carpe & 4 & 2,9 & 0,4 & 0,4 & & & & & 2,9 & 0,4 & 0,4 \\
\hline \multicolumn{12}{|l|}{ Métacarpe } \\
\hline Phal. main & 10 & 3 & 0,4 & 0,4 & & & & & 3 & 0,4 & 0,4 \\
\hline Tot. memb. sup. & 31 & 25,4 & 3,7 & 3,3 & & & & & 25,4 & 3,6 & 3,2 \\
\hline \multicolumn{12}{|l|}{ Coxal } \\
\hline Fémur & 4 & 15,4 & 2,2 & 2 & & & & & 15,4 & 2,2 & 2 \\
\hline Patella & 1 & 2 & 0,3 & 0,3 & & & & & 2 & 0,3 & 0,3 \\
\hline Tibia & 2 & 16,3 & 2,4 & 2,1 & & & & & 16,3 & 2,3 & 2,1 \\
\hline \multicolumn{12}{|l|}{ Fibula } \\
\hline Tarse & 1 & 0,3 & 0,04 & 0,04 & & & & & 0,3 & 0,04 & 0,04 \\
\hline Métatarse & 1 & 2,3 & 0,3 & 0,3 & & & & & 2,3 & 0,3 & 0,3 \\
\hline Phal. pied & 3 & 0,8 & 0,2 & 0,1 & & & & & 0,8 & 0,1 & 0,1 \\
\hline Tot. memb. inf. & 12 & 37,1 & 5,4 & 4,8 & & & & & 37,1 & 5,3 & 4,7 \\
\hline MTC MTT P hal. & & 20,6 & 3 & 2,7 & & & & & 20,6 & 2,9 & 2,6 \\
\hline membre indét. & & 420,5 & 61,3 & 54,9 & & 14,5 & 78 & 71,4 & 435 & 61,7 & 55,3 \\
\hline Total membres & 1391 & 503,6 & 73,4 & 65,8 & 41 & 14,5 & 78 & 71,4 & 518,1 & 73,5 & 65,9 \\
\hline Total déterminé & 2093 & 686,5 & 100 & 89,6 & 54 & 18,6 & 100 & 91,6 & 705,1 & 100 & 89,7 \\
\hline indét. et es quilles & & 79,4 & & & & 1,7 & & & 81,1 & & 10,3 \\
\hline Total indét. & & 79,4 & & 10,4 & & 1,7 & & 8,4 & 81,1 & & 10,3 \\
\hline TOTAL & & 765,9 & & 100 & & 20,3 & & 100 & 786,2 & & 100 \\
\hline
\end{tabular}

A
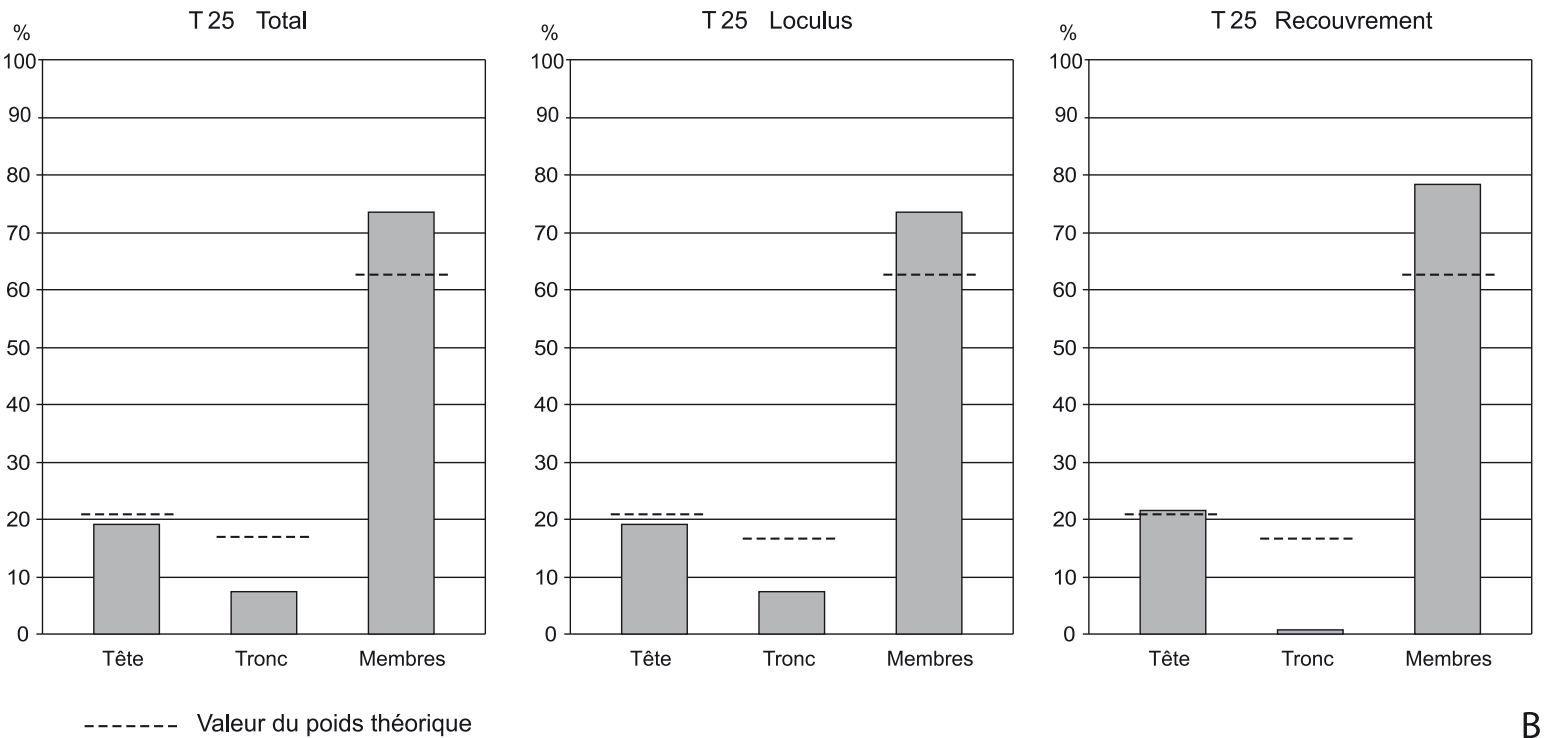

Fig. 42. Tombe 2T5. A : tableau de détermination des os humains (poids en grammes; calcul des \% sur la masse). B : répartition du poids des os par grandes régions anatomiques (\% par rapport au total déterminé). 


\section{Mobilier du loculus}

Objets personnels, armement et fourniment :

- Petit rivet de fixation d'ailettes d'umbo de bouclier, en fer ; tête discoïde, non décorée (diam. : 12 à 14 mm) ; tige de section quadrangulaire, repliée à angle droit, déterminant une épaisseur de $8,5 \mathrm{~mm}$ pour la planche du bouclier (fig. $43, n^{\circ} 1$ ) ;

- Petit rivet de fixation d'ailettes d'umbo de bouclier, en fer, semblable au précédent (diam. : 13 à $14 \mathrm{~mm}$ ); l'extrémité de la tige manque (fig. $43, \mathrm{n}^{\circ} 2$ );

- Petit fragment de bord de fourreau d'épée, en fer; gouttière avec bord de plaque; long. cons.: $13 \mathrm{~mm}$ (fig. $43, n^{\circ} 3$ ).

Objets métalliques divers :

- Applique tronconique en fer à bélière dont l'extrémité est cassée; diam. : $50 \mathrm{~mm}$; il s'agit peut-être d'une applique de ceinture ou de harnachement (fig. 43, $\left.\mathrm{n}^{\circ} 4\right)$;

- Maillon de chaînette en fer, de forme ovale, ouvert, en ruban aplati ; axes : $9 \mathrm{~mm}$ sur $11 \mathrm{~mm}$; haut. : $4 \mathrm{~mm}$; ép. moyenne : $1,5 \mathrm{~mm}$ (fig. 43, $\mathrm{n}^{\circ}$ 5) ;

- Deux fragments d'une petite tige en fer recourbée, de section quadrangulaire; long. cons. : 17 et $14 \mathrm{~mm}$; ép. moyenne : $2 \mathrm{~mm}$ (fig. $43, \mathrm{n}^{\circ} 6$ ) ;

- Fragment d'un ruban plat, en fer recourbé ; long. cons. : $12 \mathrm{~mm}$; larg. moyenne : $4 \mathrm{~mm}$; ép. : $1 \mathrm{~mm}$ (fig. 43, $\mathrm{n}^{\circ} 7$ ) ;

- Trois fragments d'un anneau en bronze incomplet ; section lenticulaire; diam. : $19 \mathrm{~mm}$. Cet objet a subi l'action du feu (fig. 43, $\mathrm{n}^{\circ} 8$ ).

Objets personnels, parure et habillement :

- Perle en pâte de verre bleu sombre, à section en « $\mathrm{D}$ », irrégulière ; diam. : $11 \mathrm{~mm}$; haut. : 3 à $5 \mathrm{~mm}$ (fig. 43, $\mathrm{n}^{\circ}$ 9) ;

- Perle en pâte de verre bleu sombre, en cours de fusion; probablement semblable à la précédente; diam. : 10 à $11 \mathrm{~mm}$; haut. : $5 \mathrm{~mm}$ (fig. 43, $\mathrm{n}^{\circ}$ 10).

Matériel céramique, portions de deux vases et tessons de vases isolés :

- Portion d'une coupe-kylix de l'atelier de Rosas, de forme Lamb. 42B (= forme Puig 33), 20 tessons représentant environ $25 \%$ du vase ; diam. ouv. : $130 \mathrm{~mm}$ (fig. 43, $\mathrm{n}^{\circ} 11$ );

- Portion (6 tessons) de l'épaule d'une urne non tournée (fig. $\mathbf{4 3}, \mathrm{n}^{\circ} 12$ ) dont la majorité des tessons se trouve à la base du recouvrement, à l'extérieur et tout près du loculus, (US 17181) (voir infra);

- 1 tesson de panse de bol de l'atelier de Rosas, de forme Lamb. 27 (= forme Puig 11).
- 2 tessons jointifs de la panse d'une autre urne non tournée (fig. $\mathbf{4 3}, \mathrm{n}^{\circ}$ 14) dont la majorité des tessons est également répartie à l'extérieur du loculus, à la base du recouvrement (US 17179) (voir infra). L'un porte la trace d'un feu secondaire, l'autre non et la différence est très notable au niveau du raccord entre les deux tessons ;

-8 petits tessons de panses de vases non tournés semblant appartenir à autant d'exemplaires (non dessinés).

Os d'animaux (détermination A. Gardeisen) :

-2 fragments de capriné brûlés, noirs (neurocrâne et diaphyse de métacarpien) ;

- 1 fragment de bourgeon dentaire de porc ;

-1 fragment de bord caudal de scapula d'animal de petite taille ;

- Indéterminé : 1 esquille non brûlée.

\subsection{La structure de recouvrement}

Dans sa partie fouillée, la superstructure de la tombe T25 comprend deux sortes d'éléments. Un certain nombre de vestiges, os, mobilier ou pierres, sont posés sur le dessus du comblement du loculus ou sur le paléosol de la nécropole, soit dispersés, soit rassemblés en trois petits groupements (US 17181, 17183 et 17179) (fig. 41, A et B). L'ensemble est recouvert par un épandage de sédiment de couleur légèrement plus sombre que sur le reste du secteur fouillé (US 1717617177-17178). Épaisse au maximum de $10 \mathrm{~cm}$, cette superstructure s'étend vers le sud jusqu'à environ $2 \mathrm{~m} \mathrm{du}$ bord du loculus, mais seulement $30 \mathrm{~cm}$ environ à l'est, ces limites restant toutefois imprécises.

\section{Groupement US 17181}

Ce groupement US 17177-17181 repose en partie sur le sol de la nécropole et en partie sur le dessus du comblement du loculus. Très serré, il se compose de trois pierres, d'une portion d'une urne non tournée écrasée sur place (fig. $43, \mathrm{n}^{\circ} 12$ ) et de deux grands morceaux de diaphyses humaines incinérées. Les pierres, du calcaire de la colline du Dévès, mesurent 12 à $14 \mathrm{~cm}$ d'arête et portent quelques traces d'éclatement au feu. Elles sont disposées selon des pendages obliques. Les tessons sont coincés entre les pierres ou appuyées contre elles. A proximité immédiate au nord, prennent place une quatrième pierre, un morceau de bûche long d'environ $10 \mathrm{~cm}$ ), qui s'est consumé sur place, et un talon de lance (fig. 43, $\mathrm{n}^{\circ} 13$ ). 


\section{Matériel du groupement US 17181}

Objet personnel, armement :

- Petit talon de lance en fer: douille conique courte, large et à pointe arrondie, formée d'un cornet simple ; chevauchement des deux extrémités de la feuille visible près du bord ; pas de trou de fixation perceptible ; long. : $69 \mathrm{~mm}$; diam. base : $20 \mathrm{~mm}$ (fig. $43, \mathrm{n}^{\circ} 13$ ).

Matériel céramique :

- Portion (25 tessons) d'une urne non tournée de forme CNT-LOR U5d, variante $\mathrm{d}$ : col tronconique rentrant, bord déversé à lèvre arrondie et méplat vers l'intérieur, panse ovoïde peignée horizontalement, épaule décorée d'un rang horizontal de légères impressions en demicercle, fond plat; diam. max. : $170 \mathrm{~mm}$. Des tessons de cette urne proviennent aussi du loculus US 17181 et du groupement US 17183 au total c'est donc environ $15 \%$ du vase qui est représenté (fig. $\mathbf{4 3}, \mathrm{n}^{\circ} 12$ ).

\section{Groupement US 17183}

Il s'agit d'une concentration assez lâche de pierres plates calcaires, de 7 à $8 \mathrm{~cm}$ d'arête, posées à plat sur le sol mais sans ordre. Ces pierres, qui proviennent également de la colline du Dévès, ne portent pas de traces d'éclatement au feu. Elles sont associées à dix petits tessons de poteries isolés :

-1 tesson d'amphore massaliète (non dessiné) ;

- 1 tesson du bord de l'urne non tournée $\mathrm{n}^{\circ} 12$;

- 1 tesson d'épaule décorée appartenant probablement à l'urne $\mathrm{n}^{\circ} 14$ du groupement US 17179 ;

-7 petits tessons de panses appartenant, au moins, à trois vases non tournés (non dessinés).

\section{Groupement US 17179}

Écrasée dans un très léger creux du sol $(1$ à $2 \mathrm{~cm}$ de profondeur), une portion de la partie inférieure d'une grande urne non tournée de forme CNT-LOR U5d, 11 tessons représentant environ $15 \%$ du vase : panse ovoïde à peignage horizontal, épaule décorée d'impressions fusiformes obliques, fond plat ; diam. max. : $220 \mathrm{~mm}$ (fig. $\mathbf{4 3}, \mathrm{n}^{\circ}$ 14).

\section{Mobilier dispersé sur le sol}

Objets personnels, parure et habillement :

- Sur le bord même du loculus, un fragment de bracelet en bronze (fig. $\left.\mathbf{4 3}, \mathrm{n}^{\circ} 15\right)$; section en « $\mathrm{D} »$; pas de décor visible ; diam. int. : $68 \mathrm{~mm}$; haut. : $8 \mathrm{~mm}$;

- À $20 \mathrm{~cm}$ du loculus, tout près de l'amas 17181 , petite fibule en fer à pied discoïde sur lequel est fixé un élément de corail (type 11 de $\mathrm{C}$. Tendille), presque complète (seul manque l'ardillon) (fig. 43, $\mathrm{n}^{\circ} 16$ et fig. 115); ressort bilatéral à six spires enroulées sur un axe en fer, non débordant ; corde externe ; arc en anse de panier, de section arrondie, légèrement épaissi dans sa partie centrale ; pied recourbé vers l'arc, dont la terminaison s'élargit en une flèche ; sur cette dernière est fixée une large plaque arrondie sur laquelle est riveté un cabochon ovale en corail, au moyen d'un rivet central ; autour de ce rivet, le cabochon est orné de trois séries opposées de demi-cercles concentriques ; long. : $38 \mathrm{~mm}$; larg. au ressort : $19 \mathrm{~mm}$; diam. du support du cabochon : $12 \mathrm{~mm}$; axes du cabochon : 8 à $12 \mathrm{~mm}$. La couleur blanchâtre du corail résulte d'une exposition au feu.

Objets personnels, armement :

- Â $1 \mathrm{~m}$ au sud du loculus, rivet (fig. $43, \mathrm{n}^{\circ} 17$ ), en fer ; tête discoïde, non décorée (diam. : $12 \mathrm{~mm}$ ); tige de section quadrangulaire, cassée à son extrémité, non repliée dans sa partie conservée (long. cons. de la tige : $12 \mathrm{~mm}$ ). Ce rivet a pu servir pour la fixation d'une ailette d'umbo de bouclier ou celle de la garniture de la poignée d'une épée.

\section{Épandage US 17176-17177-17178:}

L'épandage US 17176-17177-17178 est moucheté de petits fragments de charbons de bois millimétriques, plus denses que sur le reste du sol de la nécropole. Il inclut de très petits fragments osseux humains incinérés très dispersés, pour un poids total, dans la partie fouillée, de $20,3 \mathrm{~g}$. Il contient aussi quatre petits tessons de panse de quatre vases non tournés (non dessinés) et quelques fragments d'os animaux.

Os d'animaux (détermination A. Gardeisen) :

- 1 fragment de diaphyse distale de métatarsien de capriné adulte, montrant des stries et impacts transverses ou obliques de découpe bouchère sur le bord latéral ;

- 1 fragment de corps de côte et 1 fragment de vertèbre thoracique d'animal de petite taille.

\section{Le niveau de sol de la nécropole}

Outre l'ouverture des loculus et la base des dispositifs de recouvrement des sépultures, la surface du sol de circulation de la nécropole est bien marquée, partout, par la présence d'objets, principalement des tessons de poteries et de petites pierres calcaires non roulées, issues de la colline du Dévès. Ces objets ne montrent aucune 


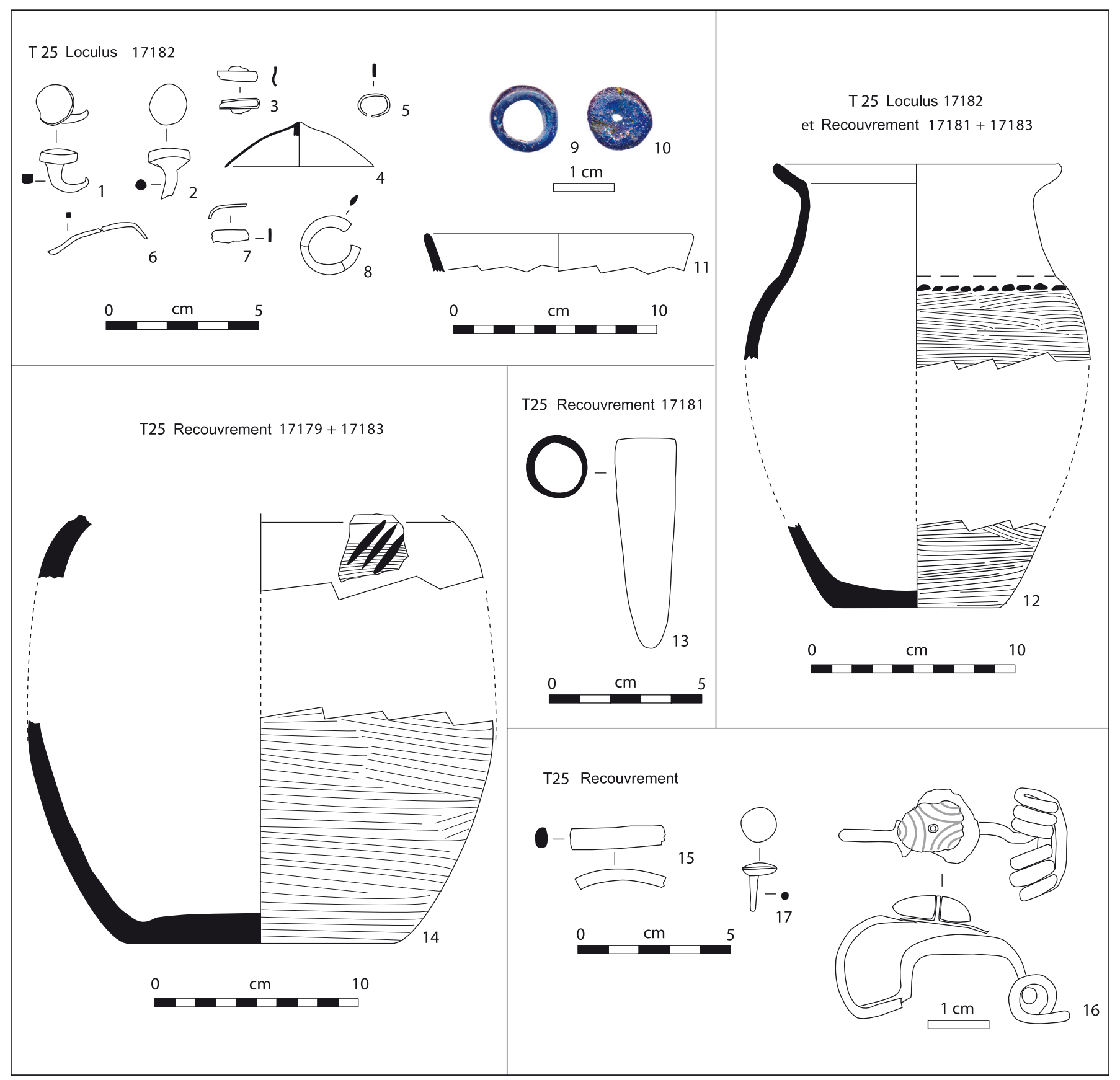

Fig. 43. Tombe 25. Mobilier (1-7, 13 et $17:$ fer ; 8 et $15:$ bronze ; 9 et $10:$ verre ; $11:$ céramique à vernis noir ; 12 et $14:$ céramique non tournée; $16:$ fer et corail).

concentration ni agencement particulier. Au contraire, ils sont dispersés et éparpillés dans les espaces entre les tombes. Tous, tessons et pierres, sont de petites dimensions. Quelques rares fragments d'os humains incinérés sont également disséminés sur ce niveau. À l'évidence ces éléments proviennent de l'érosion des structures de recouvrement des tombes. Dans l'inventaire, on suivra un ordre par secteur topographique.

\subsection{Abords de la tombe T25 (US 17176)}

Objets mobiliers :

- céramique à vernis noir : 1 bord de bolsal attique de forme AT-VN 532-561; 11 petits tessons informes de vases de l'atelier de Rosas (non dessiné) ;

- amphore massaliète : 3 petits tessons de panse (non dessiné) ; 
- céramique non tournée : 71 morceaux de panses de plusieurs urnes et coupes (non dessiné).

Os d'animaux (détermination A. Gardeisen) :

-1 fragment de vertèbre cervicale (cf. boeuf).

\subsection{Abords de la tombe T17 (US17102 et 17111)}

Os humains incinérés :

- membres : 3 fragments de diaphyses $(7,1 \mathrm{~g})$.

Objets mobiliers :

- céramique non tournée :

- fragments de cols tronconiques rentrants et bords déversés de 6 urnes (fig. 44, $\mathrm{n}^{\circ} 1$ et 2); 1 fragment d'épaule décoré d'impressions au peigne (fig. 44, $\mathrm{n}^{\circ} 4$ ) ; 34 morceaux de panses peignées de plusieurs urnes; 1 fond plat d'urne à plan de pose peigné, comme la panse (fig. 44, $\mathrm{n}^{\circ} 5$ ); tous ces tessons semblent se rapporter à des urnes de forme CNT-LOR U5d ;

- 1 tesson de jatte non tournée de forme CNT-LOR J1e, variante 1 : panse arrondie convexe, embouchure évasée, bord à lèvre élargie avec aplatissement horizontal ; diam. ouv. : $270 \mathrm{~mm}$ (fig. 44, $\mathrm{n}^{\circ} 3$ );

- 44 tessons d'urnes ou de coupes.

- dolium indigène : 5 tessons ;

- amphore massaliète : 1 morceau de panse ;

- chenet modelé en terre cuite : 1 fragment de corps (fig. $44, n^{\circ} 6$ ).

Os d'animaux (détermination A. Gardeisen) :

- 2 fragments de diaphyse de métatarse de capriné (cf. mouton).

\subsection{Entre les tombes T15, T16, T17 et T18 (US 17024 et 17034)}

Os humains incinérés :

- crâne : 1 fragment $(0,1 \mathrm{~g})$;

- membres : 21 fragments de diaphyses $(8,1 \mathrm{~g})$;

- indéterminé : 1 fragment $(0,1 \mathrm{~g})$.

Objets mobiliers :

- céramique non tournée :

- 10 fragments de cols et bords déversés, 31 morceaux de panses peignées et 1 fond plat se rapportant à au moins 5 urnes de forme probable CNT-LOR U5d (fig. 44, $\mathrm{n}^{\circ} 7$ et 8 ) ;

- 2 tessons de 2 grandes coupes de forme CNTLOR C2: panse arrondie convexe, embouchure rétrécie, bord convergent à lèvre aplatie ou arrondie (fig. 44, $\mathrm{n}^{\circ} 9$ et 10) ; 1 fond annulaire de grande coupe (non dessiné).

- céramique à vernis noir : 2 fragments informes de vases de l'atelier de Rosas; 1 bord de coupe Lamb. 27 en campanienne A ou vernis noir de Rosas.

Os d'animaux (détermination A. Gardeisen) :

- 1 corps de talus droit de capriné, très altéré (juvénile?).

\subsection{Abords de la tombe $T 12$ (17024)}

Os humains incinérés :

- crâne : 13 fragments $(2,2 \mathrm{~g})$;

- tronc : 3 fragments de côtes et 1 de vertèbre $(0,3 \mathrm{~g})$;

- membres : 82 fragments de diaphyses $(8,8 \mathrm{~g})$.

Os d'animaux (détermination A. Gardeisen) :

- 1 fragment d'extrémité proximale d'os long de bœuf (cf. humérus), altéré ;

-1 fragment de diaphyse de métacarpe de capriné, altéré ;

- Indéterminé : 1 esquille.

\subsection{Au sud des tombes $\mathrm{T} 12, \mathrm{~T} 13$ et $\mathrm{T} 14$ (US 17103 et 17105)}

Os humains incinérés :

- crâne : 1 fragment $(0,2 \mathrm{~g})$;

- membres : 21 fragments de diaphyses $(5,6 \mathrm{~g})$.

Objets mobiliers :

- céramique non tournée :

- 3 cols et bords, 5 morceaux de panses peignées et 1 fond plat d'urne de forme probable CNT-LOR U5d (fig. 44, $\mathrm{n}^{\circ} 11$ à 13 ) ;

- 1 tesson de coupe de forme CNT-LOR C1 : panse arrondie convexe, embouchure évasée, bord à lèvre aplatie (fig. 44, $\mathrm{n}^{\circ}$ 14) ;

- 1 tesson de grande coupe de forme CNT-LOR $\mathrm{C} 2$ : panse arrondie convexe, embouchure rétrécie, bord

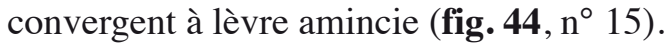

- céramique à pâte claire massaliète : 1 fond annulaire de coupe à bande peinte ocre rouge (fig. 44, $\mathrm{n}^{\circ} 16$ ) ;

- amphore massaliète : 1 petit morceau de panse ;

- chenet modelé en terre cuite : 1 morceau d'extrémité arrière (fig. 44, $\mathrm{n}^{\circ}$ 17).

Os d'animaux (détermination A. Gardeisen) :

-1 fragment d'extrémité articulaire de scapula de bœuf ; 


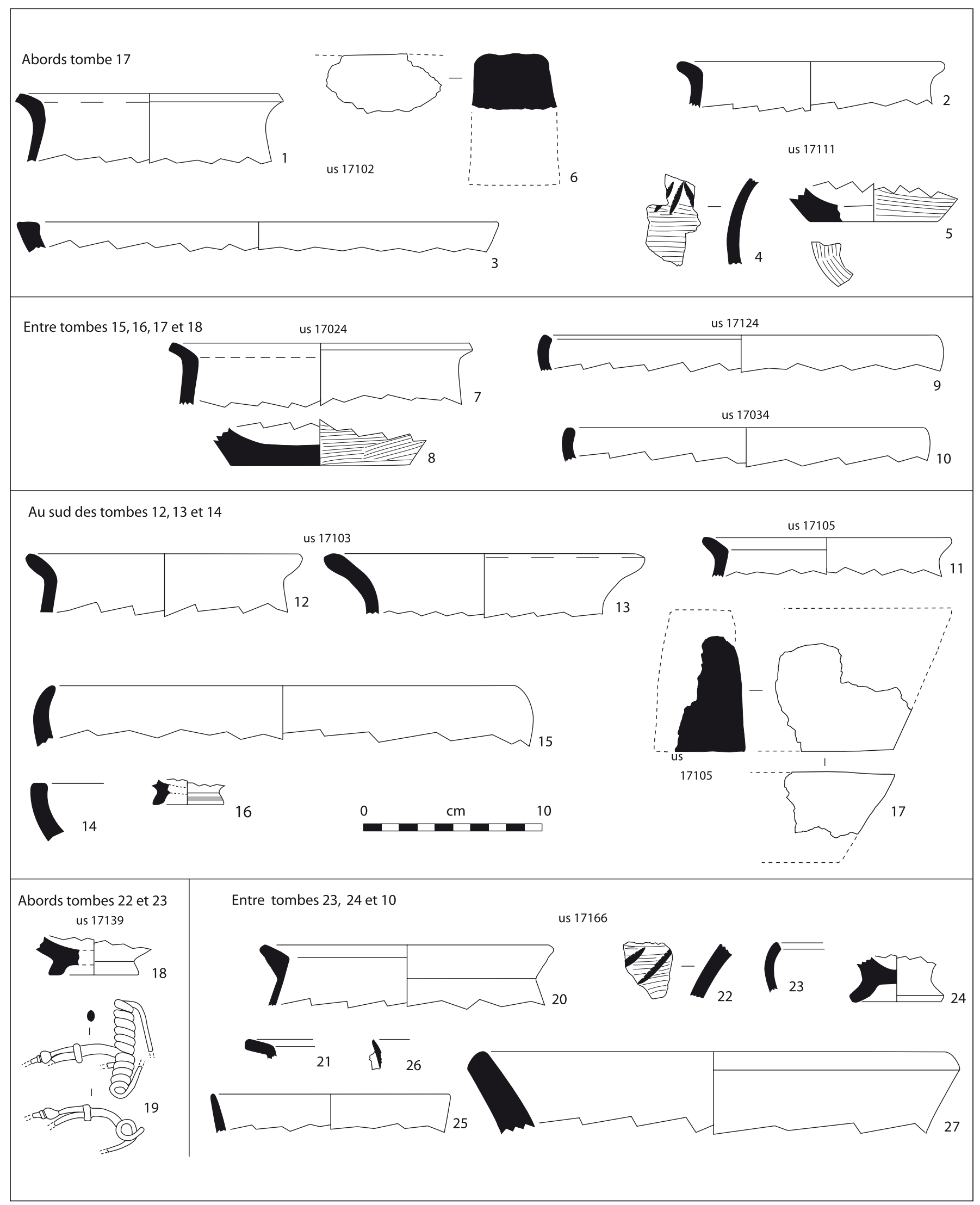

Fig. 44. Sol. Mobilier (1-5, 7-15, 20-24 : céramique non tournée ; 6 et $17:$ chenets modelés en terre cuite ; 16 et 26 : céramique à pâte calire massaliète ; 18 et 25 : céramique à vernis noir ; $27:$ mortier massaliète ; $19:$ fer). 
- 1 fragment de bord de corps de côte (cf. bœuf) ;

-2 fragments de vertèbre (cf. mammifère de petite taille);

- Indéterminés : 5 esquilles.

\subsection{Abords des tombes $\mathrm{T} 22$ et $\mathrm{T} 23$ (US 17135, 17139 et 17141)}

Os humains incinérés :

- crâne : 5 fragments $(0,7 \mathrm{~g})$;

- membres : 4 fragments de diaphyses $(0,5 \mathrm{~g})$.

Objets mobiliers :

- Petite fibule en fer, incomplète (il manque la partie antérieure du pied et le porte-ardillon) (fig. 44, $\mathrm{n}^{\circ} 19$ et fig. 115); long ressort bilatéral à onze spires et corde externe; arc en tige mince de section lenticulaire, légèrement épaissi vers le centre; grand pied recourbé dont il ne reste que l'extrémité, fixée au sommet de l'arc par deux petites griffes enveloppantes ; près de l'arc, le pied est décoré d'un petit nodule en bulbe d'oignon précédé d'une mouluration ; long. cons. : $46 \mathrm{~mm}$; larg. au ressort : $37 \mathrm{~mm}$; Type 23 de R. Gebhard (1991);

- céramique non tournée : 25 tessons d'urnes ou de coupes ;

- céramique à vernis noir : 1 fond de coupelle à vernis noir de l'atelier de Rosas, peut-être de forme Lamb. 24-25 (= forme Puig 1 ou 2); diam. base : $50 \mathrm{~mm}$ (fig. 44, $\left.\mathrm{n}^{\circ} 18\right)$;

- amphore massaliète : 1 petit morceau de panse.

Os d'animaux (détermination A. Gardeisen) :

-1 troisième molaire presque entière de porc adulte jeune, d'environ deux ans (début d'usure) ;

- 1 fragment de molaire de porc adulte, usée ;

- 1 troisième molaire inférieure droite de capriné, très usée (DMD : 23,1 mm ; DVL : 9,0 mm).

\subsection{Entre les tombes $\mathrm{T} 23, \mathrm{~T} 24$ et $\mathrm{T} 10$ (US 17166)}

Os humains incinérés :

- crâne : 7 fragments $(1,2 \mathrm{~g})$;

- tronc : 2 fragments de côtes $(0,5 \mathrm{~g})$;

- membres : 10 fragments de diaphyses $(2,1 \mathrm{~g})$;

- indéterminé : 1 fragment $(0,1 \mathrm{~g})$.

Objets mobiliers :

- céramique non tournée ;

- 2 cols et bords et 1 fragment d'épaule d'urnes de forme probable CNT-LOR U5d; l'épaulement est décoré d'impressions au peigne sur le peignage de la paroi (fig. 44, $\mathrm{n}^{\circ} 20$ à 22) ;

- 1 tesson de coupe de forme CNT-LOR C2 : panse arrondie convexe, embouchure rétrécie, bord convergent à lèvre amincie (fig. 44, $\mathrm{n}^{\circ} 23$ ) ;

- 1 fond annulaire de couvercle (fig. 44, $\mathrm{n}^{\circ} 24$ );

- 58 tessons d'urnes ou de coupes.

- céramique à vernis noir de l'atelier de Rosas : 4 tessons d'un bol de forme Lamb. 31 (= forme Puig 33) et 8 tessons informes (fig. $\left.\mathbf{4 4}, \mathrm{n}^{\circ} 25\right)$;

- céramique à pâte claire massaliète : 1 bord de coupe CL-MAS 433, avec départ d'anse horizontale (fig. 44, $\left.\mathrm{n}^{\circ} 26\right)$;

- mortier massaliète : 1 bord (fig. 44, $\mathrm{n}^{\circ} 27$ ).

Os d'animaux (détermination A. Gardeisen) :

- porc: 1 fragment de diaphyse distale d'humérus droit (adulte), 1 corps de première incisive inférieure gauche, 1 fragment de racine d'incisive supérieure ;

- capriné : 1 fragment de diaphyse de radius ;

- bœuf: 1 fragment d'extrémité proximale de métatarse ;

- Indéterminés : 8 esquilles ;

- Malacofaune : fragment de coquillage.

\subsection{Abords de la tombe T11 (US 17104)}

Objets mobiliers :

- parure:

- 1 petite perle en pâte de verre bleue, à section en « $\mathrm{D}$ » (diamètre 5 à $6 \mathrm{~mm}$ ) (fig. 45, $\left.\mathrm{n}^{\circ} 1\right)$;

- un morceau d'un bracelet en bronze, à $60 \mathrm{~cm} \mathrm{au}$ sud-ouest du loculus; peut-être ouvert, car le ruban s'amincit vers une des extrémités ; section en « $\mathrm{D} » ;$ pas de décor visible; haut. : 6 à $9 \mathrm{~mm}$; ép. : $5 \mathrm{~mm}$; diam. int. : environ $55 \mathrm{~mm}$ (fig. 45, $\mathrm{n}^{\circ} 3$ ).

- habillement :

- 1 arc de fibule en bronze, à sommet élargi en forme de losange, de section plate (long. cons. : $47 \mathrm{~mm}$; largeur : $23 \mathrm{~mm}$ ) ; cet objet est déformé par l'action d'un feu secondaire (fig. $\left.\mathbf{4 5}, \mathrm{n}^{\circ} 4\right)$;

- 1 passant en fer ; formé d'une plaque fine, grossièrement ovale, prolongée aux deux extrémités par deux pattes repliées, de section quadrangulaire ; long. : $27 \mathrm{~mm}$; larg. : $11 \mathrm{~mm}$; à $1 \mathrm{~m}$ au nord du loculus, (fig. $45, \mathrm{n}^{\circ} 2$ ).

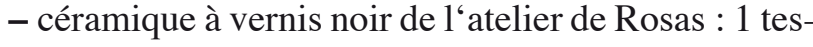
son de bord de bol de forme Lamb. 27 (= Puig 11.b) (fig. 45, $n^{\circ}$ ) et 1 petit tesson informe ;

- céramique de la Côte catalane : 1 anse et carène de gobelet de forme COT-CAT Gb0 (fig. 45, $\mathrm{n}^{\circ}$ 6) ; 


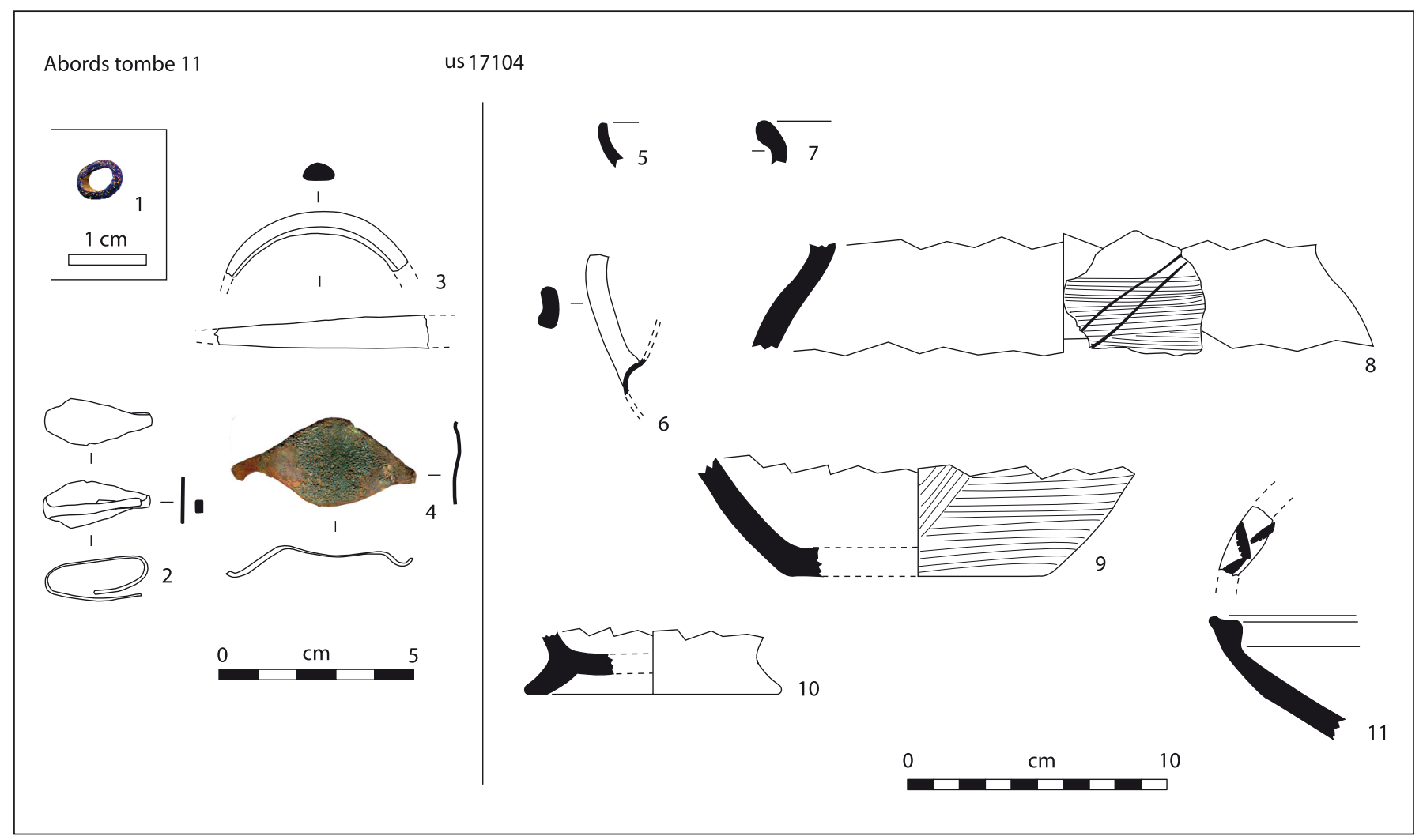

Fig. 45. Sol. Mobilier ( 1 : verre $; 2$ : fer $; 3$ et 4 : bronze ; $5:$ céramique à vernis noir ; $6:$ céramique de la Côte catalane ; 7-11: céramique non tournée) .

- céramique non tournée :

- 1 bord déversé, une partie supérieure de panse peignée avec décor de deux lignes incisées largement, peut-être double ligne brisée, 16 morceaux de panses peignées et 1 fond plat d'urnes de forme probable CNTLOR U5d (fig. 45, nº 7 à 9) ;

- 1 fond annulaire de coupe (fig. 45, $\mathrm{n}^{\circ} 10$ ) ; 1 bord de jatte portant des impressions au peigne disposées en dents de loup, et localement épaissi (fig. 45, $\mathrm{n}^{\circ} 11$ ) ; 7 tessons d'urnes ou de coupes ;

- dolium indigène : 1 tesson.

Os d'animaux (détermination A. Gardeisen) :

- 1 fragment de dent jugale inférieure d'équidé ;

- 1 troisième phalange d'équidé aux bords émoussés (mesures de la facette articulaire DT max. : 45,0 mm ; DAP max. : 22,0 mm) ;

- 1 fragment de diaphyse distale de fémur (bord caudal) d'un herbivore de grande taille, peut-être un équidé (sous réserve);

- 1 fragment de canine de porc femelle adulte ;

- capriné : 1 deuxième prémolaire inférieure gauche, 1 quatrième prémolaire inférieure droite, 1 deuxième molaire inférieure droite peu usée (jeune adulte) ;

- 1 fragment d'os crânien de grand herbivore ;
- Indéterminés : 9 esquilles de diaphyses d'os longs divers.

\subsection{Quel statut pour ces vestiges du sol de circulation?}

La présence de ces restes divers et dispersés sur l'aire de circulation entourant bûcher et tombes, avant que les limons d'inondation du Vidourle ne scellent l'ensemble, peut avoir deux origines.

L'érosion des structures de recouvrement des tombes, qui, peu épaisses semble-t-il, contiennent des éléments semblables, en est responsable pour les restes osseux humains incinérés, les rares objets de parure et d'habillement, et, sans doute, pour une part au moins des autres objets. Mais la fréquentation et l'activité des vivants durant la période d'utilisation du lieu à des fins funéraires a dû aussi générer divers déchets, périssables ou non, dont peuvent témoigner certains objets, en particulier des tessons de vases, et les os d'animaux, ou du moins une part d'entre eux. Ce dernier aspect a été évoqué pour la nécropole de la Porta Nocera à Pompéi (Lepetz, Van Andringa 2008, p. 121-122). 